\title{
Adapting an Exposure and Response Prevention Manual To Treat Youth Obsessive-Compulsive Disorder and Comorbid Anxiety Disorder: The Case of "Daniel"
}

\author{
ALEXANDER M.B. TICE ${ }^{a, b}$ \\ ${ }^{a}$ Kennedy Krieger Institute, Johns Hopkins University School of Medicine, Baltimore, MD \\ ${ }^{\mathrm{b}}$ Correspondence regarding this article should be sent to Alexander M.B. Tice, 9810 Patuxent Woods Drive Suite J, \\ Columbia, MD 21046. \\ Email: alexandertice@gmail.com
}

\begin{abstract}
Exposure and Response Prevention (E/RP) is an evidenced-based, short-term (12-20 sessions) treatment for Obsessive-Compulsive Disorder (OCD). This treatment has been shown to be effective in randomized control trials (RCTs), including pediatric samples (Franklin et al., 2011). Despite the strong evidence supporting the effectiveness of E/RP, the current E/RP literature remains wanting in terms of clinical process research to support novice clinicians in the adaptation of the principles, techniques, and interventions with such a complex and heterogeneous disorder, as well as with youth suffering from comorbid disorders. Thus, the present study aims to provide a detailed account of the course of a 25-session E/RP treatment and its outcomes with a 14-year-old called by the pseudonym "Daniel," to protect his identity. Guided by the Pragmatic Case Study Method (Fishman, 1999, 2005, 2013), as the therapist I examined this case in systematic qualitative detail and also gathered and analyzed data from standardized quantitative assessment measures. The study concludes with an analysis of the process used in meeting Daniel's positive treatment goals as well as a discussion of the importance of adaptations made to the E/RP manualized protocol to address the entire range of Daniels's symptoms.

Key Words: Cognitive-Behavior Therapy (CBT); Exposure and Response Prevention (E/RP); ObsessiveCompulsive Disorder (OCD); Generalized Anxiety Disorder (GAD); manualized treatment; case study; clinical case study
\end{abstract}

\section{CASE CONTEXT AND METHOD}

\section{The Rationale for this Particular Client for Study}

This case study aims to examine the clinical application of a manualized, evidence-based treatment for Obsessive-Compulsive Disorder (OCD), with comorbid Generalized Anxiety Disorder (GAD). It will also analyze how the application of this evidence-based treatment led to positive outcomes for a teenager struggling with complex and severe OCD and GAD symptoms, particularly by examining how treatment in the real world ultimately differed from the manualized protocol. 
To overcome his debilitating anxiety, the client, "Daniel," completed an extended course of Cognitive-Behavioral Therapy, specifically Exposure and Response Prevention (E/RP), an empirically supported treatment model for children, adolescents, and adults (March \& Mulle, 1998). Daniel was selected for this case study because his symptoms and presentation represent a common profile among adolescents with OCD in the United States. Daniel's core obsessions and compulsions involved contamination and washing, one of the most common symptom presentations for youth OCD (Flament \& Cohen, 2000). In addition, the severity of his anxiety, including significant functional impairment, presents an opportunity to analyze which interventions proved most effective or relevant to the eventual reduction in his symptoms.

Furthermore, given that adolescents with a primary OCD diagnosis have as high as a $60 \%$ chance of having a secondary diagnosis of another anxiety disorder, this case study will examine how clinicians can adapt or expand an OCD-focused exposure treatment to include noncompulsive worries (Flament \& Cohen, 2000). Treatment of anxiety in the real world, with real children and their real families, often requires a clinician to step outside the confines of sessionby-session protocols. Manuals are a critical tool for clinicians of all levels, however, they are not without flaws, sometimes lacking sufficient detail to guide a clinician in specific intervention delivery. Daniel's case was such an example, requiring considerable flexibility and creativity to ensure he could sufficiently access and tolerate the techniques to achieve a positive treatment outcome. As such, over the course of treatment, we often strayed from the manual, taking into account pragmatic changes needed to best suit Daniel, his symptoms, and his functioning.

The strategic adaptations made to the session-by-session protocol represent an important thread throughout the six-month treatment period. Given the gap between the principles of E/RP and what treatment actually looks like, particular language and techniques needed to be applied for the treatment of the identified patient. These changes greatly improved Daniel's acceptance of the treatment and his willingness to persist despite the challenges he faced as a result of his severe symptoms. Moreover, the changes, in the context of the entire arc of treatment, allowed us to examine strategies that could benefit the delivery of adolescent OCD treatment. Broadly, some of the major adaptations included: increased session frequency, the use of video technology for remote sessions, increased frequency of in-session parental involvement, and additional scaffolding pre- and post-session to increase critical reflection on therapy progress.

Through a systematic single-case study design, this article aims to address the following questions:

1. What adaptations must be made to a pediatric Exposure and Response protocol in order to achieve an optimal treatment outcome for an adolescent with OCD?

2. How can a therapist best maintain flexibility while also ensuring fidelity to the core principles of $\mathrm{E} / \mathrm{RP}$ ?

3. What additional scaffolding would benefit novice or intermediate clinicians in terms of their accurate conceptualization and delivery of E/RP? 
Pragmatic Case Studies in Psychotherapy, http://pcsp.libraries.rutgers.edu

Volume 15, Module 1, Article 1, pp. 1-74, 03-22-19 [copyright by author]

4. How can a therapist best address non-compulsive worries in the context of severe obsessions and compulsions?

\section{The Clinical Setting at Which the Case Took Place}

Daniel received treatment at the Child \& Adolescent OCD, Tic, Trich, and Anxiety Group (COTTAGe), a specialty anxiety outpatient clinic affiliated with the University of Pennsylvania's academic medical center. COTTAGe focuses on the treatment and research of pediatric anxiety and other disorders, such as body-focused repetitive behaviors. Additionally, COTTAGe provides students and practicing professionals with the opportunity to receive training in the use of evidence-based, cognitive-behavioral (CB) techniques and interventions for anxiety and other disorders. Doctoral students, such as I, gain in-depth experience in youth anxiety treatment as clinical externs working with clients on a sliding scale, under the supervision of licensed psychologists; Dr. Martin Franklin, the clinic's director, oversaw my training.

As an outpatient specialty clinic, many of COTTAGe's clients have greater access to resources and care, for example private insurance, compared to anxious youth who present to primary care offices or community mental health clinics. However, a number of COTTAGe's clients also present with quite severe or complex symptoms presentations, often having failed to achieve successful symptom reduction in previous treatment settings. Thus, parents who identify as desperate and struggling families seek out the clinic's services, sometimes traveling significant distances to the greater Philadelphia area from Southeast Pennsylvania, Southern New Jersey, and Delaware.

\section{The Methodological Strategies Employed to Enhance the Study's Rigor}

In addition to utilizing an evidenced-based treatment model for youth OCD, a number of strategies were also utilized to improve the delivery and adoption or internalization of the model, techniques, and interventions to best match Daniel's specific anxiety symptoms. First, I completed detailed clinical notes before, during, and after Daniel's sessions, that included: 1) in- depth, 3) critical reflections on the specific session, and more broadly on the conceptualization and interventions, after each session. My clinical note taking structure and process will be described in greater detail in further sections (Chapter VI). To add to the available qualitative and quantitative information about the ongoing effectiveness of particular interventions, the client also completed detailed monitoring of his progress with treatment intervention tasks on a daily basis, using a free online word processing tool that was always available to client, family, and clinician. The client's ongoing, self-report of his anxiety level (his “temperature" from 1-10) during various exposure tasks also provided a significant amount of information regarding his symptoms and experience during treatment.

In addition, audio recordings and complete transcriptions were taken for all sessions and used to analyze the entire course of treatment in order to identify effective and ineffective treatment elements. Furthermore, a number of standardized measures were employed. First, to assess for OCD in Daniel, Daniel and his family completed the Child Yale-Brown Obsessive Compulsive Scale (CY-BOCS; Goodman et al., 1989a, 1989b) at various points throughout 
treatment (see Table 1). Second, to assess for GAD in Daniel, Daniel completed ratings on the Severity Measure of GAD-Child Age 11-17, DSM-5 (American Psychiatric Association, 2018) at Session 2 and at Session 23 (see Table 2). And third, the Anxiety Disorders Inventory Schedule for the DSM-IV (ADIS-IV; Albano \& Silverman, 1996) - a semi-structured clinician-rated evaluation interview - was administered at the beginning of treatment to assess two types of anxiety problems: OCD (see Table 3) and GAD (see Table 4).

Note that the CY-BOCS was the primary quantitative measure of Daniel's progress during treatment, describing the severity of his obsessions and compulsions. It has been shown to be a consistently valid and reliable quantitative measure of youth obsessive and compulsive symptoms (Scahill et al., 1997).

\section{THE CLIENT}

The client, "Daniel," is a 14-year-old Caucasian male, living with his biological parents and two dogs in a suburban town outside Philadelphia, PA. He has two adult half-siblings from his father's previous marriage, who live elsewhere. The patient was brought to COTTAGe due to escalating anxiety, compulsive behaviors, and school refusal. Much of the patient's difficulty stemmed from severe worries about contamination, resulting in frequent hand washing and persistent avoidance of any potentially contaminated stimuli.

The parents reported a significant increase in the severity of his symptoms in the months leading up to their referral to COTTAGe, with a corresponding worsening impact on his functioning resulting from these symptoms. In particular, the patient's absences from school, and general avoidance of leaving the home, forced the family to transfer the patient to an online, home-based schooling program administered through his public school district. Unfortunately, this new school setting reduced his regular contact with same-aged peers, limiting his social interactions to more infrequent in-person contact with neighborhood friends. In terms of treatment history, the patient had minimal experience with therapy prior to treatment at COTTAGe. The parents indicated that the patient had only one lifetime course of outpatient psychological treatment at a general outpatient mental health clinic. However, the clinician at this setting did not have expertise or skills to work specifically with youth OCD treatment, terminated treatment after a few sessions, and provided a referral to COTTAGe. The family could not remember how many sessions they had attended at the prior setting.

\section{GUIDING CONCEPTION WITH RESEARCH AND CLINICAL EXPERIENCE SUPPORT}

\section{Obsessive-Compulsive Disorder: Definitions}

OCD is a complex and heterogeneous disorder characterized by obsessions - distressing and recurrent intrusive thoughts, images, or urges that persist despite efforts to ignore or suppress them-as well as compulsions - repetitive or ritualistic actions performed as efforts to reduce their distress. An individual's specific obsessions and compulsions vary significantly depending upon their age, history, life experiences, and any number of other variables. 
According to the Diagnostic and Statistical Manual of Mental Disorders, 5th Edition, (DSM-V; APA, 2013), a number of specific criteria must be met for a full diagnosis of OCD, foremost that the individual experiences obsessions and/or compulsions (see Table 3). The DSMV defines obsessions as: "Recurrent and persistent thoughts, urges, or images that are experienced, at some time during the disturbance, as intrusive and unwanted, and that in most individuals cause marked anxiety or distress. The individual attempts to ignore or suppress such thoughts, urges, or images, or to neutralize them with some other thought or action (i.e., by performing a compulsion)." In contrast, compulsions are: "Repetitive behaviors (e.g., hand washing, ordering, checking) or mental acts (e.g., praying, counting, repeating words silently) that the individual feels driven to perform in response to an obsession or according to rules that must be applied rigidly." The individual's overt behaviors or covert mental acts are "aimed at preventing or reducing anxiety or distress, or preventing some dreaded event or situation; however, these behaviors or mental acts are not connected in a realistic way with what they are designed to neutralize or prevent, or are clearly excessive."

The obsessions or compulsions consume considerable time each day (e.g., consume multiple hours per day) or cause clinically significant distress or impairment in social, occupational, or other important areas of functioning. In addition, the symptoms must not be attributable to substance use, nor better explained by another disorder or medical condition. It is important to note that many individuals with OCD possess dysfunctional beliefs, including: a need to control their thoughts, overestimating threat or danger, inflated sense of responsibility, perfectionism, low or intolerance of uncertainty, and an over-importance of thoughts. In this last case, many individuals with OCD believe, to varying degrees, that having a forbidden or "bad" thought is as bad as acting upon that thought.

Finally, insight into the disconnection between their behaviors and feared outcomes varies significantly based on a number of factors, such as age, symptom severity, and developmental level. For example, children and adolescents can often struggle to pinpoint the intended goal of their repetitive behaviors (i.e. reduce anxiety) or specifically what they fear beyond a vague feeling that something bad will happen. Based on the range of insight, the DSM-5 provides the following specifiers: with good or fair insight ("Recognizes OCD beliefs are definitely or probably not true or that they may or may not be true"), with poor insight ("Thinks OCD beliefs are probably true"), and with absent insight/delusional beliefs ("Completely convinced that OCD beliefs are true").

Recent epidemiological studies suggest that approximately $1 \%$ to $4 \%$ of children and adolescents meet criteria for a pediatric (ages 0-18) OCD diagnosis in the United States, depending upon the study (Zohar, 1999). While specific prevalence rates vary slightly depending upon the sampled country, OCD can be found across cultures (Flament \& Cohen, 2000).

Moreover, the onset of the disorder is typically gradual, although acute onset has been reported, and roughly a quarter of all OCD cases have onset by the age of 14 (Higa-McMillian, Francis, \& Chorpita, 2014). 
Gender also factors into the development of OCD. Men have an earlier age of onset compared to women, with about $25 \%$ developing symptoms by age 10 , meaning boys represent a greater proportion of youths with OCD (Higa-McMillian, Francis, \& Chorpita, 2014). Although many clients describe symptoms tracing back to early adolescence, youth OCD can often go untreated or unnoticed due to the covert nature of obsessions, the disorder's gradual development, and the fact that many children and adolescents can attempt to conceal behaviors or only present with them at home or in the presence of close family.

However, despite any hiding or minimizing, obsessive and compulsive symptoms can be extremely distressing and interfere with functioning across domains.

Many individuals also struggle with the symptoms of other comorbid disorders concurrently with OCD, and at various stages throughout their lifetime. For example, many children and adolescents struggle with the symptoms of other anxiety disorders (e.g. social anxiety disorder, separation anxiety disorder, panic disorder, etc.), as well as mood disorders (e.g. major depression), and tic disorders. In fact, some studies suggest lifetime comorbidity rates as high as $75 \%$ for those suffering from OCD (Zohar, 1999). Generalized Anxiety Disorder and Panic Disorder appear to be one of the most common comorbidities with OCD, consistent with Daniel's GAD comorbidity (Zohar, 1999).

Most OCD youth experience multiple obsessions and compulsions, with fear content that can fluctuate (Flament \& Cohen, 2000). This can mean greater variability in symptoms for children, compared to adults, whose symptoms often remain more stable over time. A number of researchers have examined the structure and patterns of OCD symptoms, identifying roughly four or five relatively independent symptom dimensions: contamination/cleaning, doubts about harm/checking, symmetry/ordering, and unacceptable/taboo thoughts (including religious/moral and somatic obsessions) (Mataix-Cols et al. 2008; Williams et al., 2008). Hoarding has also been included as a fifth category, although it is important to distinguish from the distinct Hoarding Disorder (Mataix-Cols et al. 2008). There have been some conflicting findings as to whether pediatric OCD has the same structure as adult OCD, although recent results point to a similar four factor structure (Mataix-Cols et al. 2008).

Furthermore, research has found that children and adolescents with contamination/cleaning symptoms tend to have higher avoidance scores on the CY-BOCS compared to other patients (Williams et al., 2008). These patients often present as "phobic-like" due to strong avoidance of possibly contaminated people or objects that is often mediated by strong disgust reactions. However, literature suggests individuals with this specific profile of symptoms tend to have favorable results with exposure-based interventions. In addition, individuals with OCD can experience what has been colloquially described as a "just right" feeling, an internal sense that things are not complete or incorrect in some way (Franklin et al., 2015). Often, an action must be repeated many times to find or achieve a particular feeling that can resolve that sense of wrongness. 


\section{Theoretical Models of OCD}

There have been significant evolutions in the understanding of OCD over the past fifty years, from theoretical models and conceptualizations to efficacious treatment interventions.

However, the etiology of OCD for any individual remains in large part unclear. As with many complex physical diseases or psychological disorders, it appears to be influenced by biological, genetic, and environmental factors, with complex, multidirectional interactions and interrelationships. In addition to those variables, psychological factors and patterns of cognitions and behaviors have been identified as contributing heavily to the disorder, and thus have been described in great detail as our models have evolved in recent decades.

One of the first models of OCD came in the form of Mowrer's (1960) Two-Stage Theory, which suggests a first stage in which a neutral object or event becomes associated with fear by being paired with a stimulus that provokes distress and anxiety. A second stage involves efforts by the individual to avoid or reduce the anxiety felt by contact with the feared objects or events, by both passive avoidance strategies and eventually ritualistic behaviors to reduce distress. These avoidance strategies then function to strengthen the anxiety and distress as a result of temporary anxiety-reduction, or a cycle of a negative reinforcement (Mowrer, 1960). However, further examination of the Two-Stage Theory revealed insufficient and equivocal support, as well as a lack of sufficient detail about the complex cognitive processes and patterns found in OCD, and suggested the need for more comprehensive models.

Salkovskis (1985) offered a more comprehensive cognitive framework for understanding OCD, arguing that an individual's intrusive obsessions act as stimuli that can provoke certain classes of negative automatic thoughts, which in turn lead to mood dysregulation if those automatic thoughts clash with the individual's belief system. For example, an individual with OCD might experience an intrusive thought or image with sexual themes, which interacts with a held belief that only "bad" people have sexual thoughts. Thus, the friction between the individual's beliefs and the intrusive thought leads to negative affect and mood dysregulation.

Salkovskis (1985) highlighted themes of self-blame and exaggerated senses of responsibility and self-blame as important in the beliefs/schema of those with OCD. In turn, both cognitive and behavioral compulsions represent efforts to remove blame and lessen that sense of responsibility.

Furthermore, Salkovkis argued that people with OCD have five distorted assumptions: 1) "Having a thought about an action is like performing the action"; 2) "Failing to prevent (or failing to try to prevent) harm to self or others it the same as having caused the harm in the first place"; 3) "Responsibility is not attenuated by other factors (e.g. low probability of occurrence); 4) Not "neutralizing" when an intrusion has occurred is similar or equivalent to seeking or wanting the harm involved in that intrusion to actually happen; 5) "One should (and can) exercise control over one's thoughts" (Salkovskis, 1985, p. 579). Based on these assumptions, treatment logically should be directed toward or focused upon identifying the distorted assumptions and beliefs, and then modifying the automatic thoughts that result from those thought patterns. 
More current cognitive theorists have built upon Salkovskis' ideas related to an individual's sense of responsibility, including Rachman's (1998) concept of thought-action fusion (TAF). Individuals with OCD often believe that simply having a thought increases the chance of the bad outcome happening, and that thoughts of doing something abhorrent are the same as having actually done something abhorrent (Rachman, 1998). Furthermore, Purdon \& Clark (2002) suggest that beliefs such as TAF, intolerance of uncertainty, and an exaggerated sense of responsibility, lead to a vicious cycle of avoidance that ultimately strengthens the OCD. In short, those beliefs may lead to ineffective attempts to stop or suppress their thoughts, which unfortunately leads to the opposite, a greater frequency of negative or obsessional thoughts and distress (Purdon \& Clark, 2002). Treatment based upon these cognitive theories would address the individual's distorted beliefs with the goal of disrupting that vicious cycle.

Although it is helpful to understand at the level of theory the complex patterns of OCD, models must also discuss the ways in which an individual's behaviors contribute to its development and maintenance. Foa \& Kozak (1985) describe an integrated model that effectively balances the role of cognitions and behaviors in anxiety, and OCD more specifically. They conceptualize fear as a network of information existing in memory, which includes stimuli, responses, and meaning. Thus, anxiety represents specific impairments in the networks of our emotional memory.

The fear networks of anxious individuals skew toward a resistance to modification, distorted threat perception, abnormally high negative valence toward feared outcomes, and exaggerated physiological responses.(Foa \& Kozak, 1985). Fear structures can include magnified connections between a stimuli and the anxious response, in addition to distorted perceptions about a stimuli and potential harm. These individuals engage in active avoidance, which contributes to the persistence of these distorted fear networks. Furthermore, some specific fears have only limited opportunities for organic exposure, limiting how much they would be challenged in daily life.

Foa \& Kozak (1985) also highlight various ways in which information processing can break down, a process which is necessary for influencing fear networks and ultimately, behavior. Breakdowns in processing can stem from inaccurate beliefs and rules of inference, cognitive defenses, and even exaggerate arousal with a lack of habituation.

Foa \& Kozak (1985), as well as others, emphasize that OCD differs from other disorders because of the ways that individuals process information. The research suggests impairments in how an individual makes inferences about harm, such as estimating danger based on the lack of evidence for safety rather than evidence of danger. Thus, compulsive behaviors intended to lessen the chance of harm cannot produce safety in themselves and therefore need to be repeated.

Based on this integrated CB model, treatments using exposure techniques could be effective in addressing areas of weakness in information processing. Imaginal exposure could influence the individual's perceptions of harm versus thoughts of harm. In contrast, in-vivo exposure could demonstrate that being in the presence of the feared stimuli does not increase the actual probability of harm, thus impacting their distorted estimates of likelihood. 
In terms of the specific manual used throughout Daniel's treatment, which we will describe in the Course of Treatment section below, the manual authors describe OCD as a "neurobehavioral disorder," emphasizing the role of a "broken" neurological response to anxiety and persistent maladaptive behavioral patterns. This formulation will be described in greater depth below as it applies to Daniel.

\section{Treatment of Obsessive Compulsive Disorder: A History of Cognitive \& Behavioral Interventions for $\mathrm{OCD}$}

Cognitive-behavioral treatments emphasizing exposure interventions are the current gold standard for OCD treatment, with strong empirical support across various settings. However, before their development and improvement, there was a dearth of efficacious OCD treatments. In the 1960s, Dr. Victor Meyer undertook the earliest applied research examining the effectiveness of the behavioral, exposure-based interventions that underlie integrated CB treatments.

Specifically, he began by utilizing exposure activities with intention prevention of rituals with two adult patients who responded well to treatment. Meyer (1966) then expanded the sample to treating fifteen adult OCD cases with his exposure-focused, behavioral techniques, which resulted in ten individuals with very successful outcomes and five with partially successful outcome. In addition, Meyer found that only two patients had relapsed at a five-year follow up.

Over time, Meyer's exposure techniques evolved to the current treatment under examination: Exposure and Response Prevention (E/RP). Many studies have replicated and improved upon Meyer's early successes, reinforcing the effectiveness of exposure interventions for the treatment of OCD in both adult and pediatric populations. These studies provide empirical support in the form of methodologically rigorous Randomized Control Trials (RCTs) as well as open clinic trials whose format may better reflect real world clinical populations and practice (Abramowitz, Whiteside, Deacon, 2006). For example, Franklin, Ambramowitz, Kozak, Levitt, and Foa (2000) compared posttreatment E/RP outcomes for randomized and non-randomized patients, finding significant symptom reductions in both groups. Across various settings and locations, ERP was found to be superior to a number of other control treatment conditions, including placebo meds (Marks et al, 1980), Relaxation (Fals-Stewart, Marks, \& Schafer, 1993), and Anxiety Management Training (Lindsay, Crino, \& Andrews, 1997).

\section{Exposure and Response Prevention}

Although the treatment has evolved from Meyer's original approach, some of the most effective aspects include his core elements of prolonged exposure to stimuli associated with the obsessions, and strategies aimed at stopping the initiation or completion of compulsions. These can be categorized as in-vivo, i.e. occurring in real time in as realistic a situation as possible, or as imaginal, i.e. mentally imagining the situation and its possible consequences. Both types of exposures aim to bring the client into contact with stimuli or cues that provoke their specific obsessions and associated distress. In practice, clinicians attempt to elicit repeatedly, over time, the individual's specific feared situations and/or thoughts to provide an opportunity to disprove and undermine their distorted perceptions, associations, and conclusions. 
In coordination with the exposure, the clinician coaches the client through response (ritual) prevention, the process of blocking rituals or reducing avoidance behaviors, such as hand washing or avoidance of feared stimuli. Many clinicians can also find that training of cognitive skills, including mental strategies designed to improve resistance to OCD, can contribute to a successful reduction in compulsive behaviors. Children and adolescent treatment protocols have also integrated a family or parent component, as research has demonstrated the importance of parental involvement and support to positive outcomes (Selles et al., 2017).

Daniel's treatment was based upon the March, Mulle, Foa, and Kozak's unpublished treatment manual, an adaption of John March and Karen Mulle's book entitled "How I Ran OCD Off My Land!” (March \& Mulle, 1994). However, successful treatment to address Daniel's specific obsessions and compulsions, as well as his non-compulsive worries, required a flexible approach that concurrently maintained fidelity to the principles and techniques utilized in the protocol. The treatment manual's authors describe four "steps" to their treatment that occur roughly in the following order: 1) Psychoeducation, 2) Cognitive training, 3) Mapping OCD, and 4) Intensive, graded exposure and response prevention.

As the first phase of treatment, psychoeducation represents the first opportunity to help a client reduce their symptoms; reviewing the appropriate and comprehensive knowledge of the symptoms, treatment model, and interventions are critical to an ultimately successful treatment. The authors emphasize educating clients on the neurobehavioral perspective of OCD, which takes the view that the disorder is a medical illness with a dysfunction of normal processes, like asthma or diabetes, that can be managed. Useful metaphors for the obsessions thus include: a broken volume control or brain "hiccups." Clinicians and clients also discuss the benefits, (minimal) risks, and the commonly required tasks of behavioral interventions for OCD, so that everyone fully understands that to which they are committing.

Finally, the authors instruct the clinician to use a technique from Michael White, an Australian family therapist, who puts the disorder in a narrative context, with story metaphors used to describe its influence on a child's life as well as the ways in which the child is already working to resist the OCD.

Cognitive Training is the second step of the protocol, although these activities occur during the first two sessions in conjunction with psychoeducation. The cognitive training is designed to improve the client's "sense of personal efficacy, predictability, controllability, and self-attributed likelihood of a positive outcome for the E/RP tasks" (March \& Mulle, 1998, p. 5). Specifically, the clinician coaches the client to deliver positive self-talk and encouragement to help bolster their own motivation and efforts to resist.

This cognitive training is accomplished in part by framing E/RP as the strategy in the battle against OCD, with the therapist and family as the patient's allies. In addition, the client externalizes the OCD by giving it a bad name, e.g. "fear monster," which casts the OCD as an "enemy" rather than more aptly as a bad habit they struggle to control. Brainstorming a nickname for the OCD would not be appropriate for every client, as many adolescents may consider it silly rather than constructive. However, the underlying principle of externalizing and distancing the 
client from his or her OCD remains an important one to impart upon the client.

Furthermore, clinicians are encouraged to describe the patient's efforts to resist the obsessions and compulsions as "bossing back" the OCD throughout the manual. If looked at differently, the OCD has been bossing them around, and so now it is the client's job to "boss it back." Finally, the therapists are continually reinforcing accurate knowledge of OCD, encouraging the patient to remind his or herself of information about OCD's insidious nature.

Step three of the treatment occurs during sessions three and four in the protocol, and aims to "map" the child's experience with OCD, including specific obsessions, compulsions, triggers, avoidance behaviors and consequences. Presenting the task as creating a map helps to structure the stimulus hierarchy in a more easily digestible format, where the patient and family can visualize their symptoms and progress. This mapping also encompasses "islands" where the child does not experience OCD, others where the OCD and patient each "win" some of the time, and finally others where the child feels OCD wins all or most of the time. The clinician also describes a "transition zone," in which the "winning" side is shifting in favor of the patient, using this as a descriptive tool to help guide the patient across graded exposures. The authors note that the transition zone often represents the lower end of the stimulus hierarchy, especially at the beginning of treatment

At the end of this phase, usually session 4, the therapist and patient will complete the first exposure activities, with easy, or only mildly anxiety provoking, "trial" tasks, seeking to assess the patient's distress tolerance, willingness to engage in exposures, and knowledge of OCD. In addition, successful completion of lower difficulty exposures can provide the patient with needed confidence that the treatment will ultimately be successful, particularly if those mastery experiences come early in the course of treatment.

The remainder of the treatment protocol, from sessions three through twelve, falls under Step Four, arguably the most important components: intensive, graded exposures and response prevention. Tasks focusing on exposure and ritual prevention represent the core activity of the treatment, and stretch across the remainder of the treatment sessions. Once the client has successfully approached and engaged with his or her obsessions, fears, or worries (i.e. exposures) without engaging in compulsive behaviors, the emphasis of treatment will shift to generalization and ultimately maintenance and relapse prevention.

Given that the authors designed the manual to be effective in the treatment of children and adolescents with OCD, the protocol emphasizes appropriate parental involvement in the efforts to support the client in symptom reduction. Each session includes parental involvement, to varying degrees, with a check-in at the beginning and/or end of each session, as well as parent-only sessions. However, this may be dependent upon the level of parental entanglement in the patient's OCD. Many parents have gradually become increasingly involved in the accommodation of their child's avoidance behaviors and/or directly involved in the rituals. Thus, they too need the relevant psychoeducation, coaching, support, and encouragement to effectively reduce their involvement. Parents can also present with their own worries regarding the patient's symptoms and/or the role of a parent, and thus therapists must consider how best to engage the parents such 
that they are an added benefit to the treatment rather than an unintended barrier.

Overall, parental engagement in the treatment, such as assisting in or monitoring exposures, increases over the course of treatment, considering that parents are often involved in rituals that are higher up on the hierarchy and therefore become targets later in treatment.

Eventually, parents can become co-therapists (or "co-soldiers" within the military metaphor), with the child's approval. This aspect of treatment requires flexibility to "meet" the patient and parents "where they are" in terms of their specific symptoms and circumstances. Parents themselves must also be flexible, and frequently it can be critical that the parents learn to reduce giving of advice or instruction for the exposures because it is important that the child build a sense of self-efficacy and that he or she is willingly and successfully engaging with the treatment techniques.

The manual provides detailed, itemized plans for each session that build upon each other, and overall, they follow a similar structure throughout treatment. Each session begins with setting an agenda, confirming or agreeing upon the intended activities with the patient to gain buy in. Next, there is a review of the prior week, which includes an assessment of symptoms, functioning, progress, and any problems that may have arisen since the previous session. The clinician introduces any new information, concepts or techniques (i.e. cognitive skills, exposure, ritual prevention, etc), which is then followed by the main event: therapist-assisted "nuts and bolts" practice. At the beginning of treatment this may mean practice with cognitive skills, but for the majority of the sessions this component represents in-vivo practice with exposure and response prevention. Before the end of each session, the clinician and client discuss and determine appropriate homework tasks, such as exposure practice, monitoring of symptoms, and ERP tasks.

The sessions for Daniel's course of treatment followed a consistent structure that prioritized in-session and homework exposures, with the goal of a continual increase in the difficulty of exposures as he moved up the hierarchy. In-vivo exposures to the feared stimuli on his hierarchy represented the primary intervention, and took up the majority of our time and focus. Based on Daniel's self-report or "temp" rating, we gradually increased the exposure difficulty while eventually establishing strict rules for hand washing only prior to meals, which he was ultimately able to maintain. Prior to beginning an exposure intervention, I provided the patient with a range of options, roughly taking the form of 1) slightly more challenging exposures, 2) more challenging, and 3) definitely more challenging than previously attempted. I also included at least one that he explicitly refused to complete in a previous session, to give him the option to jump up the ladder. I encouraged him to do the hardest exposure he could, while reinforcing the rationale for doing so. I always participated in the exposures alongside him, doing everything he did. Finally, throughout all exposures I prompted for his "temperature" or "temp," as well as the feared consequences.

\section{Cognitive-Behavioral Therapy (CBT) Mechanisms of Change}

Underlying the early CBT theoretical models and treatment of OCD was Emotional Processing Theory (EPT), which suggests that exposure treatments are effective because they 
work to break an individual's conditioned fear responses by way of habituation. The theory proposes that exposure activities interact with the "fear structures" that exist in a given patient's memory, leading to information that conflicts with that fear structure (Jacoby \& Abramowitz, 2016). In addition, the incompatible experience or association is then considered to be integrated into the individual's fear structure by way of "corrective learning" to the extent that non-fear based elements can replace existing fear associations. Within this model, habituation is directly tied to E/RP change as evidence of that corrective learning. Habituation can be understood as a "short-term, sensory effect" that leads to significant reduction in the intensity of an individual's reaction to a stimulus (Jacoby \& Abramowitz, 2016). More concretely described, the patient would react with less fear to anxiety-provoking stimuli with repeated contact to those stimuli.

Although this was the dominant theory for explaining symptom reduction and improvements in functioning for considerable time, further research over the past few decades has pointed to the limitations of this widely accepted theory. Lang (1971) suggested it is important to monitor the change in fear reactions based on three response symptoms: verbal, behavioral, and physiological. Foa \& Kozak's (1986) view has gained popularity, and it points to three signs of emotional processing when looking to monitor for a successful outcome: initial fear activation (how anxious the client became during the exposure), within-session habituation, and between-session habituation. Furthermore, Foa and colleagues argue that between-session habituation depends upon within-session habituation, and therefore represents the better avenue for longer-term learning.

Foa \& Kozak's understanding of how exposures influence fear directly impacts how therapists explain and implement treatment. Under this model of change, therapists describe the rationale for treatment as that prolonged and repeated exposure leads to fear reduction during the session and between the sessions. Moreover, sessions are structured or extended to allow habituation, because exposures only end when habituation is achieved. It is understandable then, that therapists focus heavily on habituation as they understand it to be linked with treatment effectiveness. Unfortunately, the literature is mixed with regard to whether habituation is a reliable predictor of the outcomes of exposure therapy: some researchers favor it, while others do not (Foa et al., 1983; Baker et al., 2010). Some studies still even suggest that habituation is not necessary for the successful response to exposure treatment (Tsao \& Craske, 2010). Due to this conflicting research base, it is important that clinicians take caution when using habituation as an outcome indicator, as it is unclear if it represents true learning. Craske et al. (2008) argue that this lack of support reflects the disconnection between true fear learning and how an individual expresses fear during learning.

Despite the best intentions, emphasizing habituation may also lead to a few unforeseen consequences. For example, a "fear of fear" mindset can develop if the patient perceives anxiety to be bad in and of itself, and/or that treatment is only successful if they are completely free of fear or anxiety. The most successful patients will be flexible in their understanding and reactions to anxiety, without over-pathologizing the experience of anxiety.

Furthermore, recent research into fear extinction, defined as a decline in conditioned fear responses after a nonreinforced exposure to a feared conditioned stimulus, suggests that a patient's 
fear associations do not disappear completely, but rather that exposures lead to learning of new non-threatening associations that exist compete with pre-existing hreatening associations (Craske et al., 2008). The non-threatening associations are considered inhibitory. These results are consistent with Bjork and Bjork (1992)'s theory that suggests associations in memory remain even with infrequent access or "use." They argue that once learned, associations don't truly fade over time, but rather that the accessing of those associations decreases over time. This is adequate for most people, but those with OCD are vulnerable to relapse due to the fear-based associations remaining in memory. This foundation eventually led to the development of the Inhibitory Learning Theory (ILT, Lang, Crakse, \& Bjork, 1999), which attempts to explain the role of extinction in the exposure process. ILT proposes that the individual's pre-existing fear/threat associations are not replaced or erased by the new non-fear/threat associations that are acquired during exposure learning. Therefore, the stimulus now has an ambiguous meaning, with both a fear and non-fear associations existing in their memory and competing for retrieval. The authors argue that the fact that fear can return following successful exposure therapy suggests that a patient's original fear/threat associations continue to remain.

This model has important implications for clinicians implementing E/RP. Specifically, clinicians should try to maximize the chance that the individual's new, non-fear/threat associations will inhibit the process of memory retrieval of older fear/threat associations (Jacoby \& Abramowitz). For example, this could include additional session time spent on generalizing learning to other settings or contexts. In addition, clinicians should emphasize fear tolerance, or "open-mindedness toward these experiences given that they are universal, inevitable, and nonthreatening" (Jacoby \& Abramowitz, 2016). Craske et al. (2008) also suggest that therapists can teach patients that fear during exposures, and fear experiences in general, represent a chance to practice tolerating or managing distress, rather than representing failure or a relapse.

\section{Generalized Anxiety Disorder: Definitions}

In addition to his primary diagnosis of OCD, Daniel also struggled with non-compulsive worries that fell within a diagnosis of Generalized Anxiety Disorder (GAD; see Table 4). The Diagnostic and Statistical Manual of Mental Disorders, Fifth Edition (DSM-5) indicates that for a child or adolescent to meet the criteria for a Generalized Anxiety Disorder diagnosis, an individual must have the following symptoms:

O excessive anxiety and worry (apprehensive expectation), occurring more days than not for at least six months, about a number of events or activities (such as work or school performance);

○ difficulty controlling their worries;

$\bigcirc$ their worries are connected to one or more of the following symptoms being present for most of the past six months:

- restlessness or feeling keyed up or on edge;

- easily fatigued; 
- difficulty concentrating or mind going blank;

- irritability;

- muscle tension; and

- sleep disturbance (difficulty falling or staying asleep, or restless, unsatisfying sleep);

○ anxious thoughts and physiological symptoms cause clinically significant distress or impairment in social, occupational, or other important areas of functioning; and

○ these symptoms must not be a result of substance use or a medical condition.

In short, GAD manifests as excessive anxiety and worry about multiple activities and/or events, although the focus of a child's worry can shift from one concern to another over the course of the disorder. The worry is out of proportion to the feared consequence's actual impact or chance of occurring. Those with the disorder also struggle to manage or control their worries, such that anxious thoughts interfere with task-oriented attention. Pediatric GAD often presents as excessive worry about punctuality, catastrophic events (e.g. earthquake, war, etc.), and performance quality or competence in scholastic or athletic events, even if that performance is without an evaluative component.

In fact, Weems, Silverman, \& La Greca (2000) found the following as the most frequently reported worries in a clinical sample of children: grades/test scores, natural disasters, being physically attacked, and being bullied by peers. Of course, children can also worry about adult concerns, like family finances. Children with GAD often worry about low-frequency events, such as being robbed, shot, or otherwise attacked, without recognizing that such events occur infrequently. They also present as unsure of themselves, perfectionist, conforming, and/or repeat tasks due to dissatisfaction with below perfect quality. They may also frequently seek reassurance or approval from others, including parents, regarding their performance or concerns about a specific feared event.

Consistent with the diagnostic criteria, a client's specific anxious thoughts are accompanied by a range of physiological and somatic symptoms (APA, 2013). Many individuals with GAD report being anxious most of their lives, although onset can occur over a wide age range. These individuals may struggle with excessive worry prior to the worsening of their anxiety, such as children with an anxious or avoidant temperament. In terms of rates of the disorder, the twelve-month prevalence of GAD is approximately $1 \%$ of adolescents in the United States (Kessler, et al, 2012), while the lifetime prevalence was approximately $2.2 \%$ of the population.

\section{Theoretical Models of Generalized Anxiety Disorder}

Although not as well understood from a theoretical perspective as other anxiety disorders, GAD can be conceptualized through a number of possible theories. For example, theories of avoidance and negative reinforcement can explain the maintenance or continuation of anxiety over time. Other theoretical examples for GAD include a revised "contrast avoidance" model of 
worry from Newman and Llera (2011), which suggests that for individuals with GAD, worry functions as a strategy to avoid a negative contrast in moods. Thus, they prefer chronic worry or distress to shifts from a positive to negative state, or from a mildly negative to a profoundly negative state. Newman and Llera argue that worry is reinforced by the relief experienced when the feared negative event does not occur. Whichever model is applied to understand the worry, psychopharmacological and psychotherapy treatments, such as exposure-based CBT, have been shown to treat GAD successfully (Higa-McMillan, Francis \& Chorpita, in Mash \& Barkley, 2014). Historically, models of pediatric anxiety have tended to focus only on one aspect, such as cognitive, learning, or behavioral theories, that were adapted from adult anxiety (Marsh \& Barkley, 2014).

As the understanding has advanced, the models have become more complex, integrating multiple contributors to the development and maintenance of anxiety disorders, including biological, psychological, and environmental factors. Pine (2009) integrated cognitive and affective neuroscience research, genetic research, information-processing concepts, and brainimaging studies in proposing his theory for understanding anxiety disorders in children. Barlow's (2002) triple-vulnerability model has also received significant coverage, as it suggests that generalized biological and psychological vulnerabilities, in tandem with specific factors in the environment, place an individual at higher risk for developing an anxiety disorder in childhood.

The strongest contemporary models acknowledge and reflect the transactional nature of anxiety development. Child specific variables (e.g. temperament, genetics) interwine with environmental variables (e.g. peers, community), and remain in dynamic interaction throughout a child's development (Vasey \& Dadds, 2001). Furthermore, factors such as parenting styles relate bidirectionally with specific child characteristics, including temperament (Edwards, et al., 2010).

Ultimately, the field of youth anxiety treatment needs to work toward comprehensive multidimensional theories of childhood anxiety that encompass the abundance of etiological contributors, including temperamental, familial, psychosocial, genetic, and neurobiological. Furthermore, these theories must reflect that the highest priority intervention targets differ according to the unique nature of the individual and context, as well as across time and the individual's development. For example, techniques focused on parenting strategies have been shown to be effective for younger children, but may lose strength as that child moves into adolescence.

A number of thought and behavior patterns appear to contribute to the ongoing reinforcement and maintenance of client's excessive, uncontrollable worry over time. These patterns include: avoidance of anxiety provoking or negative thoughts, images or situations (negative reinforcement), difficulty relaxing, a tendency to repeat worries instead of problem solving, and dysfunctional patterns of thinking about the utility of worrying (i.e. overestimate the value of worrying). Similar to OCD and other anxiety disorders, avoidance is a core component of GAD and must be gradually reduced over time via imaginal and in-vivo exposure to their worries. 
Pragmatic Case Studies in Psychotherapy, http://pcsp.libraries.rutgers.edu

Volume 15, Module 1, Article 1, pp. 1-74, 03-22-19 [copyright by author]

\section{CBT for $G A D$}

Similar to OCD treatment, cognitive behavioral techniques to treat GAD have evolved, building upon successes with adults while also adjusting for the differences reflected in the presentation of childhood anxiety. Current cognitive and behavioral treatment for GAD can refer to a wide range of possible techniques that can be delivered individually or in some combination. Consistent with the overarching cognitive behavioral concepts, thoughts, feelings and behaviors are interrelated, therefore changing one's negative thinking patterns can lead to a reduction in anxiety, for example.

GAD therapy would also emphasize cognitive restructuring, or the effort to challenge and adjust the patient's often catastrophic thoughts and beliefs. These techniques might also try to challenge the GAD patients' commonly held belief that worrying serves a useful function in their lives, so they should continue with the status quo despite some negative impact. Behavioral techniques would include controlled exposures to anxiety-provoking or avoided situations (and

thoughts), relaxation training, and even the scheduling of specific, limited periods of time in which they are "allowed" to worry.

Overall, there is significant overlap in the techniques and interventions utilized to reduce OCD and GAD symptoms, given the notable similarity of those disorders. For example, exposure activities can be implemented to help person learn that feared outcomes do not come true, and to experience a reduction in anxiety over time (be it habituation or inhibitory learning). Unlike $\mathrm{E} / \mathrm{RP}$, however, CBT for GAD would include time spent on cognitive restructuring, or challenging the client's distorted, anxious thinking patterns, as well as relaxation training activities. Cognitive restructuring can often be challenging with OCD, or even contraindicated, due to the intolerance of uncertainty, while relaxation can sometimes be utilized in a ritualistic manner that interferes with treatment.

\section{Overlap and Interaction Between OCD and GAD}

Many children and adolescents present with symptoms of multiple disorders, particularly when struggling with anxiety, and therefore treatment must appropriately encompass all presenting issues. For example, in Masi et al.'s (2004) clinical sample of 157 youth with GAD, depression was found to be the most common, account for 56\%; OCD was present in $19.7 \%$ of the sample. In other research, Storch et al. (2007) examined the impact of various comorbid psychiatric disorders on CBT outcomes for childhood OCD, in a sample of 96 patients, aged 7 to 19 , at an academic treatment center. Nearly $75 \%$ of their sample met criteria for a comorbid disorder, and important to this current study, the presence of a comorbid disorder negatively affected treatment response. As a starting point of comparison, 92\% of the sample without a comorbid disorder were treatment responders and achieved clinical remission of their symptoms. With one or more comorbid diagnoses, only $69 \%$ were treatment responders and only $46 \%$ met criteria for symptom remission. 
Storch et al. (2007) also argue that this may be due to the simple fact that comorbid disorders naturally consume some portion of session time focused on OCD-related tasks and techniques. However, despite these largely negative results, the decrease in treatment response and remission rate differed depending upon the comorbid condition, such that the presence of ADHD, disruptive behaviors, and depression had the greatest negative impact. Comorbid anxiety disorders on the other hand, specifically GAD, did not appear to have such a significant impact on treatment response and remission rates. Given the authors' simple argument that greater time spent on exposures leads to greater symptom reduction, it is understandable that treatment outcomes would not be as negatively impacted. Considering both disorders are in the anxiety family it is unsurprising that both would respond to similar interventions.

\section{Cognitive Behavioral Therapy Versus Psychopharmacology}

Independent of the cognitive and behavioral interventions described so far, a variety of antidepressant, serotonergic medications have also been shown to produce clinically significant symptom reductions in children and adults suffering from OCD, including sertraline, clomipramine, fluoxetine, and fluvoxamine (March et al, 1998; POTS I, 2004; Franklin et al., 2003). To better understand the range of treatment options for children and adolescents, the Pediatric OCD Treatment Study I (POTS I, 2004) compared the efficacy of three treatment conditions: A. Medication (sertraline), B. CBT, and C. Medication \& CBT) to a pill placebo control condition (Franklin et al., 2003). This multi-site, open clinical trial recruited sample of 112 youth aged 7 to 17 with a primary diagnosis of OCD, with roughly equal age and gender distribution. The researchers' Intent to Treat analyses resulted in significantly better treatment outcomes for all three treatment conditions compared to pill placebo. In addition, the combined medication \& CBT treatment was superior to CBT or sertraline alone, conditions that did not have significant outcome differences.

However, the authors note that roughly $54 \%$ of patients in the combined treatment and $39 \%$ of those in the CBT only condition met criteria for excellent clinical response, which was defined specifically as a post-treatment CY-BOCS total score of 10 or lower. These rates of "excellent clinical responders" in the combined and CBT conditions compared to roughly $21 \%$ in the sertraline condition and $3 \%$ in the placebo condition. Thus, while the combined condition did outperform the CBT-only condition, both of those conditions significantly outperformed medication-only and placebo conditions. Despite the evidence base for the efficacy of CBT in the treatment of OCD, most pediatric OCD patients that present to treatment from the community receive treatment in the form of a serotonin reuptake inhibitor (SRI). (Franklin et al., 2003).

One meta-analysis of the efficacy of pharmacological treatments for GAD examined the rates of response to SSRIs, SRNIs, and benzodiazepines, suggesting that upwards of $60 \%$ to $75 \%$ of patients will see sharp symptom reductions (Baldwin, Ajer, \& Garner, 2009). In fact, the results of numerous studies suggest that a significant reduction in symptoms will be more likely with a combination of therapy and medication, notably as the severity of symptoms increase (Abramowitz, Whiteside \& Deacon, 2006). Medication can help to reduce symptoms sufficiently to allow patients to engage fully in the behavioral treatment tasks. However, it is important to consider the pros and cons of medications, as well as the preferences of the child and family. 
Some families do not want to medicate their children or face any number of possible side effects (although these are often mild). In addition, some families fear that medications must be continued in order to see sustained results, or they may struggle with the expense or consistent administration of the medication.

In comparison, cognitive behavioral therapy adaptations to the treatment of OCD and GAD can produce profound, long-term results depending upon on the quality of intervention implementation, patient commitment, and learning (Franklin et al., 2015). However, treatment requires significant time and resources, as well as the completion of numerous energy-consuming and emotionally-distressing activities. Of course, a great strength in the treatment is that a successful course of therapy provides the client with knowledge and skills that can be utilized in the future in the case of symptom reemergence or relapse.

\section{ASSESSMENT OF THE CLIENT'S PRESENTING PROBLEMS, GOALS, STRENGTHS, AND HISTORY}

\section{Presenting Problems}

As with every patient at COTTAGe, Daniel and his parents participated in a comprehensive diagnostic evaluation to gather information on presenting problems, symptoms, and functioning. To facilitate accurate differential diagnosis, we completed two semi-structured clinician-led interviews: the Anxiety Disorders Interview Schedule for DSM-IV (ADIS-IV) and the Children's Yale-Brown Obsessive-Compulsive Scale (CY-BOCS). As it happened, I was assigned to his evaluation, prior to being assigned his case for treatment. The ADIS-IV was used as a diagnostic assessment tool to confirm diagnoses at treatment onset only. In terms of his endorsed symptoms, Daniel's parents reported that he had been experiencing significantly impairing anxiety, as well as related compulsions, since age seven. Daniel reported severe worry about contamination, such as coming into contact with germs or other "dirty" surfaces, believing this would lead to him or someone else falling sick. Relatedly, he also experienced anxiety about his health and safety, as well as that of his parents. After some psychoeducation regarding obsessions, Daniel acknowledged the intrusive, unwanted nature of his thoughts. He reported worry about no specific contaminate or illness, but rather about getting sick in a general sense, as well as a concern about throwing up.

In addition, with corroboration from his parents, Daniel reported spending more than eight hours a day obsessing about contamination and other worries, causing severe distress because of their frequency and disturbing nature. Daniel also reported yielding to all of his obsessions without attempting to control them, albeit with some mild reluctance. Finally, Daniel had little control over his obsessions because he was rarely successful in stopping them, often only able to divert his attention away with great difficulty. As for his compulsive behaviors, both Daniel and his parents endorsed significantly impairing cleaning rituals related to his contamination fears, which included: excessive hand washing, showering, and avoidance of possibly contaminated items or surfaces. Daniel felt that he spent approximately three to eight hours per day engaged in washing his hands, cleaning himself, or avoiding contaminated stimuli (see Table 1, Table 3, and 
Figure 1).

Daniel also endorsed experiencing clinically significant worries that were not linked to compulsions, and the evaluation confirmed he also met criteria for Generalized Anxiety Disorder (GAD). He endorsed elevated worry about social situations/interactions, in particular that others will notice and judge his anxiety, leading to a negative evaluation. In addition, he endorsed worries about performance (academic grades) and world events (e.g. recent terrorist attacks in Paris). Daniel felt different from his peers because he believed he worries far more than others (see Table 4).

Furthermore, Daniel also experienced a high level of sensitivity and hypervigilance toward physiological sensations, particularly feeling nauseous prior to and during school. He and his parents believed these symptoms most likely stemmed from his anxiety. These physiological sensations often make it difficult for him to attend a full day of school, if he was willing to go at all. Daniel also described discomfort with the sensation of feeling full, which also led to worrying about vomiting, and thus he often restricted how much he ate. This eating restriction paralleled a greater pattern of behavioral avoidance of anxiety provoking stimuli. For example, complete avoidance of crowded places was linked to contamination fears, worries about being judged negatively, as well as the possibility of throwing up due to anxiety. Finally, Daniel and his parents denied any history of trauma, symptoms of psychosis, mania, and they reported no other psychiatric symptoms. He also denied thoughts of self-harm and suicidal ideation. Table 5 presents Daniel's diagnoses at the beginning of treatment, utilizing the DSM-IV-TR axis system.

\section{Relevant Background Information}

Daniel resides in a suburb of Philadelphia, Pennsylvania, with his mother, father, and three dogs. He also has an older brother (age twenty-six) and an older sister (age twenty-three), who both live outside of the home. The patient reported having a good relationship with parents and brother, but that he does not get along well with his older sister. He has a small group of friends at school, and a best friend, but he does not feel he needs to make any more friends. While discussing interpersonal relationships, he endorsed worry about social situations and interactions with people with whom he is not as comfortable.

In surveying the family history, the father endorsed a past history of possible OCD symptoms, specifically with counting and arranging compulsions. The father reported that he had never engaged in treatment himself, but that his symptoms had decreased in severity since his early twenties. Daniel's mother also described herself as having an anxious temperament, including worry about Daniel. Finally, the parents endorsed other forms of mental illness on both sides of the patient's extended family, but were unwilling to describe this history in depth.

\section{Quantitative Assessment: Children's Yale-Brown Obsessive Compulsive Scale (CY-BOCS)}

In order to reliably assess Daniel's obsessions and compulsions, the clinician-administered the Children's Yale-Brown Obsessive Compulsive Scale (CY-BOCS), which was used as the primary measure throughout treatment. The CY-BOCS is a semi-structured interview that 
provides a detailed understanding of an individual's current, past, unique fears and compulsive strategies to manage their distress. It is widely considered the gold standard for OCD assessment, in part for its depth and clinical utility, as well as its high reliability and validity (Goodman et al., 1989a; Goodman et al., 1989b). According to a recent analysis of a pediatric OCD sample, it would be appropriate for clinicians to consider remission to have occurred when their pediatric patients' CY-BOCS raw total score falls below the clinical cutoff score of 11 (Skarphendinsson et al., 2016). This clinical cutoff is similar to that of other recommendations that recommend a post-treatment total score of 10-12.

The administration encompasses both patient self-report of symptoms and clinician observation. First, the clinician guides the patient through a comprehensive checklist of common obsessions and compulsions. Next, the clinician guides the patient through a rating of the severity of his or her obsessive symptoms on a range of critical dimensions, grounding them with the following prompt: "I am now going to ask you questions about the thoughts you cannot stop thinking about."

The severity of the patient's obsessive and compulsive symptoms are measured across multiple dimensions, including Time Occupied by Obsessions/Compulsions, Interference due to Obsessions/Compulsions, Distress Associated with Obsessions/Compulsions, Resistance Against Obsessions/Compulsions, and Degree of Control Over Obsessions/Compulsions. Both the Obsession and Compulsion symptom severity scores range from 0 to 20 and combine for a "Total Severity Score" that ranges from 0 to 40 . The authors provide the following descriptive classifications for various ranges of symptoms: Extreme (32-40), Severe (24-31), Moderate (1623), Mild (8-15), and Subclinical (0-7).

To monitor his symptoms over time, Daniel completed the CY-BOCS at the intake evaluation, start of treatment, and at various point throughout the treatment (Session 1, 7, 13, 19,23, and 24). At the initial evaluation, Daniel received a total score of 31 (Obsession severity=16, Compulsions $=15$ ), which indicates symptoms that fall in the Severe Range for their frequency, intensity, and interference (see Table 1). He endorsed obsessions related to the following concerns: dirt, germs, sticky substances/residues, and getting others ill by spreading contaminants. He also described engaging in the following compulsions: excessive washing, showering, asking his parents repeated questions, and checking for contaminants. He described very frequent intrusive thoughts, resulting distress, and only limited symptom-free periods of time. Confirming the reason for referral, Daniel's symptoms met criteria for a current diagnosis of Obsessive-Compulsive Disorder, with fair insight (see Tables 1 and 3). In addition, Daniel completed ratings on the Severity Measure of GAD (Child Age 11-17, DSM-5) at Session 2, and received a 3.2 (out of 4), which fell into the severe range (See Tables 2 and 4).

\section{Diagnoses at Treatment Onset}

Daniel's diagnoses at the onset of treatment included:

○ Primary: 300.3 (F42.2) Obsessive Compulsive Disorder

○ Secondary: 300.02 (F41.1) Generalized Anxiety Disorder 


\section{Strengths}

Daniel has a very close relationship with his parents, and his strong support network represented an important factor in the positive treatment outcome. His parents were committed to supporting Daniel as much as possible, and utilized their flexible work schedules to great effect. In addition, Daniel and his parents brought a positive and open attitude to every interaction, even when Daniel struggled with his symptoms. Furthermore, Daniel maintained a support group of neighborhood friends throughout his experience with OCD and treatment. Despite Daniel's persistent avoidance, his friends continued to search him out, which provided additional motivation and positive reinforcement of his ongoing efforts.

Daniel is bright and performed well academically when his symptoms did not interfere.

His interests include video games and playing with his friends and with his dogs. When comfortable, Daniel is fun-loving and enjoys humor. Further, his efforts and commitment over the course of treatment reflected that he is determined and hard-working.

\section{CASE FORMULATION AND TREATMENT PLAN}

\section{Case Formulation}

The extensive assessment of Daniel's anxiety resulted in a detailed understanding of his specific obsessions and related compulsions, as well as his non-compulsive worries. Consistent with the "neurobehavioral framework" presented in the March \& Mulle (1998) treatment manual, Daniel's primary OCD is understood at its most basic level as a neurological issue that has developed over time as a result of a variety of factors. Although the development of these issues in Daniel's brain cannot be easily teased apart at this time, theories of classical conditioning may explain some part of the development of his worries and obsessions. For example, he may have learned to associate once neutral objects with a feeling of fear, such as a school desk with fears of contamination. Thus, the conceptualization explicitly avoids assigning blame to anyone, including patient or parents. Rather, his anxiety is described with alarm or computer metaphors as a "short circuit," "hiccoughs," and/or a "volume control problem," in which he receives "false alarms" related to danger or threat.

Over time and as the fear associations strengthen, operant condition could explain the evolution of his compulsions, as his various escape and avoidance behaviors escalated to cope with his worries. Specifically, Daniel engaged in compulsive washing, which had the powerful, short-term effect of rapidly reducing his distress after contacting a contaminated object.

However, the temporary reduction in distress is greatly overshadowed by the cycle of negative reinforcement that ultimately only functions to strength his worries and maintain distressing obsessions about contamination. In short, typical with OCD, Daniel's use of rituals to cope with his obsessions began to spiral out of control to the point to which he could only cope using those rituals, rather than with other, more adaptive strategies. 
March \& Mulle's (1998) "neurobehavioral framework" emphasizes behavioral components, but Foa \& Kozak (1985) also provide important insights into the distorted cognitions that characterize Daniel's obsessions. First, his cognitions regarding contamination reflect that he assigns significantly higher probability of danger to situations that are safe: that desk must be contaminated, or that doorknob is most likely going to get me sick if I touch it.

Furthermore, he had a tendency to exaggerate the severity of his feared outcome, such that becoming sick would amount to something terrible or intolerable. Finally, many individuals suffering with OCD, including Daniel, seek constant evidence of safety because in their minds, situations must be dangerous if they do not have direct proof of security. These patterns of cognitions, including his specific worries and his perceptions of those worries, play an important role in the conceptualization, as they impact how his escape and avoidance behaviors (rituals) manifest.

In addition to his OCD symptoms, it also appeared that Daniel had developed escape and avoidance behaviors related to non-OCD worries. For example, Daniel often worried about peer judgment of his anxiety, which contributed to his school refusal. Even when in class, Daniel avoided the attention and eye contact of teachers and peers. Thus, Daniel developed strategies to avoid anxiety-provoking stimuli as much as possible, which served a similar function as his washing compulsions by negatively reinforcing his worry over the long-term by only temporarily reducing his negative affect. It is important to note how Daniel's worries and related escape/avoidance behaviors overlap significantly in terms of his OCD and GAD symptoms.

Many feared stimuli were connected to both obsessions and other, non-OCD worries, such as a school classroom being associated with fears of contamination and negative evaluation. Overall, a vicious cycle develops for individuals like Daniel, involving avoidance behaviors that maintain and strengthen obsessions and other worries.

It is also critical to consider the influence of other aspects of his history and how they relate to the origins and maintenance of his symptoms. The development of Daniel's anxiety has most likely been influenced by multiple factors, including genetic vulnerability and a predisposition to an anxious temperament or strong physiological fear reactions, but his learning history also appears to be a contributor. As previously mentioned, Daniel's father endorsed a history of OCD while his mother described herself as also experiencing anxious thoughts and feelings. It seems reasonable to conclude that Daniel's parents modeled anxious responses as well as escape and avoidance strategies for dealing with distress; as a result, Daniel may have learned over time to engage in maladaptive behaviors that independently reinforced his anxiety.

Separately, Daniel's parents engage in a pattern of accommodation and shared escape/avoidance related to Daniel's maladaptive beliefs and behaviors. This included allowing Daniel to avoid feared situations, like going to school, or picking him up early, as well as following various rules about the cleanliness of objects in the home. Much like Daniel's, their behaviors reduced the chance that natural extinction would take place and maintain his symptoms through avoidance and negative reinforcement. Also, it may be that these accommodation behaviors are linked to the precipitants of the current episode's severity. 
Approximately three to four months prior to contacting COTTAGe, Daniel's parents reported a spike in the severity and impact of his anxiety, especially in relation to the number of classes and school days missed. This increase in symptom severity coincided roughly with the beginning of the patient's $7^{\text {th }}$ grade school year. However, as stated above, a multitude of factors contributed to the development of Daniel's symptoms, and it is important to avoid assigning blame or attempting to come to a rational understanding of why his anxiety developed in the way it did. Although patients often wish for a tidy picture of why they are suffering in such a way, it can be counterproductive to go too far down those paths.

\section{Treatment Plan and List of Treatment Goals}

Based on the theoretical models underlying Daniel's case formulation, CBT interventions were the most appropriate treatment, with Exposure and Response Prevention (E/RP) indicated to treat his OCD. To guide the treatment, I used the March, Mulle, Foa \& Kozak protocol that is an adaptation of March \& Mulle (1994) book “How I Ran OCD Off my Land!” an empirically supported treatment for youth OCD (Abramowitz et al., 2005) that is recommended as a first line treatment due to its demonstrated efficacy. Due to the severity and interference of his OCD symptoms, the treatment first began with a focus on symptom reduction (Abramowitz, Franklin, \& Foa, 2002). Once Daniel's OCD symptoms had decreased sufficiently and when it appeared clinically appropriate, we began to gradually include treatment of his non-OCD worries.

Although we did not utilize any particular treatment manual, the treatment of his nonOCD anxiety also emphasized the use of graded exposures to feared stimuli, along with cognitive interventions as needed, consistent with exposure principles.

Given Daniel's presentation, his treatment goals were the following:

1. Reduce frequency of compulsive behaviors;

2. Learn to tolerate or cope with anxiety and distress; and

3. Improve functioning, including increasing engagement in developmentally appropriate tasks inside the home and social activities outside of the house.

\section{COURSE OF TREATMENT}

\section{Clinical Note Taking}

As mentioned previously, I established additional scaffolding and structure related to the treatment process that aimed to enhance my critical reflection and thinking about the formulation and intervention. This additional thinking and note-taking enabled continual updates to the conceptualization, as well as optimal individualization and adaptation of the protocol to be most effective at targeting Daniel's specific obsessions, compulsions, and other worries. 
Prior to each session, I created an Agenda/Plan to determine the most appropriate sequence of tasks and activities (see Table 7 for an example of the Agenda/Plan from Session 9). Depending upon the phase of treatment, this included the following frequently occurring items: (a) Review of the past week, including a brief assessment of anxiety and any events that may have impacted; (b) Review of the assigned homework, including the encouragement, reinforcement, and problem-solving of obstacles; (c) Psychoeducation, including the introduction of new concepts and/or reinforcement of previously discussed concepts; (d) Exposure practice; (e) Planning of homework exposures and ritual prevention; and (f) Working with the parents on topics like parental psychoeducation, a review of Daniel's therapy homework, and a review of the treatment sessions.

For the two components of the weekly homework, exposure and ritual prevention, Daniel was instructed to keep a detailed monitoring record of each day, using a free application similar to Microsoft Excel ("Google Sheet"; see Table 8 for an example). This online record form included columns to track various aspects of both (a) Daniel's exposure tasks (exposure procedure and goal time, Temp 1-10, successful at "bossing back," Y/N resisted longer than goal, notes about exposure); and (b) Daniel's ritual prevention tasks (Ritual Prevention: Goal \%, OCD "wins”, D "wins", \% D won). Using this online tool provided a number of benefits not seen with pencil and paper, such as preventing loss of the record and allowing me to stay updated with Daniel's progress across a given week. Daniel's parents also had free access to the record, enabling them to also participate in monitoring and oversight. We could also adjust that form to suit our purposes as much as needed, and it also allowed his parents to be able to chime in with sometimes critically useful comments and notes about his progress.

Consistent with best practices, I also took down detailed notes about the therapy process, based upon my process notes taken during session as well as my review of session audiotapes (see Table 9 for an example). My post-session critical reflections represented one of the most important components of the added structure because it prompted regular, repeated, critical thinking about the conceptualization as well as application and delivery of various E/RP interventions. I would typically spend 5-10 minute after each session (and prior to completing the clinic's EMR documentation) reflecting upon what had been accomplished in session, updating the formulation with new assessment information, and considering how to adapt techniques and interventions for future sessions. These periods of reflection frequently generated constructive and important questions for individual and group supervision.

In addition to providing a valuable opportunity to consider his symptoms and how to creatively target them, this additional note taking process led to more relevant and constructive supervision with my supervisor, Dr. Franklin. As will be discussed in the Phase sections below and in the Discussion section later on, it can be critical, especially for children and adolescents, to find the right language to describe the complexities of OCD and E/RP in ways that they can understand and translate into action. Dr. Franklin is an expert as finding those metaphors, analogies, and mental images that most resonant with pediatric patients, and thus, my additional time spent on critical thinking led to a very useful to honing of how I communicated concepts to Daniel. Specifically, I described OCD and E/RP through the lens of "starving versus feeding the OCD," "leaning in versus leaning away," as well as the metaphors of video games and sports. 
Pragmatic Case Studies in Psychotherapy, http://pcsp.libraries.rutgers.edu

Volume 15, Module 1, Article 1, pp. 1-74, 03-22-19 [copyright by author]

\section{Phase I: Assessment (Sessions 1 \& 2)}

Prior to diving into treatment, it was important to gather an updated assessment of Daniel's symptoms and functioning in order to develop a systematic case formulation and treatment plan for him. Thus, we devoted the first two sessions to completing a detailed clinical interview as well as a repeated administration of the CY-BOCS. Unlike the initial evaluation, we were able to focus entirely on his OCD and GAD symptoms, allowing a more in-depth discussion of his worries. To build rapport, Session 1 began with an informal conversation about his interests and life. Daniel expressed an interesting in video games and playing with his dogs, but appeared nervous and was relatively quiet. We transitioned to completing the CY-BOCS, which enabled a detailed dissection of his symptom presentation. Similar to the initial evaluation, in the formal assessment Daniel required psychoeducation about obsessions and compulsions in order to fully understand and respond to my questions. Moreover, the psychoeducation was helpful because Daniel's full understanding of OCD would be critical to the upcoming E/RP treatment. Daniel had some difficulty expressing the content of his obsessions as well as distinguishing compulsive and non-compulsive anxiety, which was further complicated by the overlap between his obsession and his non-OCD worries.

Despite somewhat limited insight, Daniel was cooperative and answered all of my questions as best as he could. He endorsed an Obsession severity score of 13, and a Compulsion severity score of 12 , for a Total Severity score of 25, which fell into the "Severe" range. This represented a considerable decrease from the Total Severity Score of 31 from the intake evaluation, and while this current rating still fell into the "Severe" range, it was on the low end rather than high. Daniel could not attribute the drop to any particular reason, but expressed he had been trying harder to resist the OCD. To capitalize on this apparent shift, I highlighted this attitude as an essential component to treatment, and encouraged him to keep it up (see Figure 1).

Session 2 involved a clinical interview aimed at gathering information regarding Daniel's non-compulsive worries. For example, he worried about the elevator ride to our sixth-floor office, because of an uncomfortable sensation in his stomach. This opened the conversation to the challenge of attending therapy and the conflict that arose with his parents. He had been anxious about the elevator and more generally about the session, and had only attended after his parents insisted. After praising him for fighting through his anxiety, we focused on increasing his motivation by exploring how OCD and anxiety impacts his life, and why he would want this to change. Daniel struggled with insight into the negative impact of his symptoms, but did want to worry less, suggesting at least some motivation. When asked about a return to school, as a possible motivator, he quickly described his dislike of school, stating that his mother agreed with him that "cyber" school was better. Daniel also completed a rating of his GAD symptoms, using the Severity Measure for GAD, a newly developed assessment tool using DSM-5 GAD criteria. He received a severity rating of 3.2 out of 4 , which fell into the severe range (see Table 2 and Figure 2). Daniel's parents joined near session's end, and we briefly reviewed the session. I asked them to keep track of his anxiety over the next week, emphasizing monitoring what triggered the obsessions and compulsions. 
Pragmatic Case Studies in Psychotherapy, http://pcsp.libraries.rutgers.edu

Volume 15, Module 1, Article 1, pp. 1-74, 03-22-19 [copyright by author]

\section{Phase II: Psychoeducation, Hierarchy Building, and Trial Exposures ( Sessions 3, 4, \& 5)}

Session 3 represented our first direct foray into E/RP, beginning at the first session in the March et al. (1998) manual with psychoeducation (see Table 10 for additional session details). Initially, we discussed the monitoring homework briefly, noting moments of worry and their associated triggers. I emphasized we would be "bossing back" the OCD, linking him to the examples from his week, and contrasting how the OCD has been bossing him around for some time. I reinforced the externalization of OCD by prompting Daniel to come up with a name for his disorder, which he named "Fear Monster." Next, I described the model's view of OCD within a neurobehavioral framework, not as a "bad habit" but rather a neurological issue. I used the metaphor of an alarm with a broken volume and sensitivity system that frequently goes off incorrectly (false alarm) with an excessively loud noise. Further, we discussed the role of compulsions in the maintenance of his obsessions, and how hand washing only temporarily reduces his distress. As Daniel highlighted, the relief only lasts for a short time before he is anxious again, which negatively reinforces his obsessions over time.

I also introduced him to the language of "feeding" versus "starving" the OCD. Every time he allows his OCD to "boss him around" and completes a compulsion, he is "feeding" the disorder and allowing it to get stronger, in contrast to "starving" it when he successfully resists the urge to ritualize. In addition, I described the two primary interventions in more depth, with exposures to confront feared triggers (e.g. touching the walls), and response prevention to stop himself from ritualizing (e.g. hand washing). I also outlined our roles, with mine as "coach," and that his family members are his "team" to help him be successful in treatment.

Since the introduction to some of this psychoeducation in the first two sessions was brief, we had sufficient time in Session 3 to begin building a hierarchy of feared stimuli (this process usually occurs in session four in the manual). This activity involved identifying and rating various possible triggers (e.g. stimuli, situations, etc) from 1 to 10 for how anxiety-provoking they each would be to him. I also often asked a clarifying question: "If I were to ask you to do this, without doing a compulsion, how hard would it be?" My supervisor, Dr. Martin Franklin, recommends using this language to directly link to the eventual exposure exercises. After spending fifteen minutes creating the hierarchy, his parents joined us and I again assigned monitoring his anxiety over the next week.

Following session 2 in the protocol, Session 4 (see Table 10) began with a review of the past week, using the following language to provide continuity from the previous session: "How has OCD bossed you around this week? How/when have you said 'no' to OCD?" We briefly reviewed the list of triggers Daniel had to create for homework, and he reported his mother had completed it but that he had been sitting with her. Setting that list aside for the later hierarchy activity, we returned to a quick psychoeducation discussion of OCD's "two flavors" from the protocol.

Specifically, I described how some people experience strong negative affect related to various stimuli (fear of harm, disgust, and/or guilt), while others experience a need for having things "just so." Finally, I encouraged him always remember to directly confront or "lean in" to 
his anxiety, rather than away, and that the more he does it, the easier it will become.

Next, we returned to finish assigning ranks to items for the hierarchy. Daniel had a better grasp on this task on the second attempt, and it became clear that the hardest items were associated with other people or were located outside of his home. As corroborated by his parents, he had been unwilling to go into any stores, restaurants, or other businesses for many months, due to overwhelming anxiety and avoidance. It also became apparent that these worries were connected to contamination obsessions, as well as worries about negative evaluation.

Once we completed the hierarchy, rating as many examples as we could brainstorm, I transitioned to discussing exposures and how we could do them in session and at home. To practice, I asked him to do a quick in-vivo exposure that involved holding my water bottle. With no reluctance, Daniel held my water bottle for a minute with both hands. Before and during, I asked for his "temperature," which he rated as a three, suggesting it was not very hard. Prompted for any feared consequences, he expressed concerns about it being clean (e.g. contaminated) and if he would get sick. After completing the trials and discussing exposure homework, he expressed willingness to try something more difficult at home, volunteering to expose himself to the dog toys, which he had rated as a five. More specifically, we created a detailed plan for completing two to three exposures on different days over the next week: touching the dog toys for one minute in each hand and then resisting washing his hands for as long as possible. His parents joined the session to confirm the trial exposure homework (and corresponding record form) and continued monitoring of his symptoms for the hierarchy.

Session 5 (see Table 10) corresponded with the third session in the E/RP manual, and began with a review of his symptoms and functioning since the last session. Despite an incomplete monitoring form, Daniel reported he had completed the trial exposures. We discussed his monitoring in order to rule out any new obsessions or compulsions, but Daniel indicated nothing new. We then reviewed his successful completion of the dog toy exposures. He again reported experiencing significantly more anticipatory anxiety prior to the exposure $(\mathrm{Temp}=7)$ compared to actually touching the $\operatorname{dog}$ toys $(\mathrm{Temp}=4)$, and that he had been able to resist washing his hands for roughly 10 minutes.

After reviewing these exposures, we returned to a final activity in the manual for the hierarchy, which aims to identify the "transition zone" of the patient's OCD symptoms. This involves identifying A) symptoms he can successfully resist, B) symptoms he successfully resists $50 \%$ of the time, and C) symptoms he is unable to resist. We then use this information to create a "map" of the "battle" with OCD to visualize any progress. I admit that I struggled with this activity, because the manual does not provide particularly in depth or concrete direction about language or how best to discuss it. Furthermore, in my post-session clinical reflection notes, I questioned its applicability to this specific case, as Daniel appeared disengaged. Unfortunately, it seemed Daniel did not fully connect with the metaphor underlying this particular activity.

We moved on to discussing the "toolkit" that Daniel can call upon for challenging exposures: the fear thermometer, positive self-talk, and rewards. This allowed us to begin the conversation about positive reinforcement, or rewards, for homework completion. The topic of 
possible motivating rewards was couched in a discussion of planning exposures, both in session and as homework. In fact, we completed a relatively brief exposure in parallel with this conversation, specifically Daniel holding the doorknob with both hands for approximately 10 seconds, with the goal of not washing his hands until he returned home. We returned to planning exposures for homework, while I periodically prompted him to provide temp ratings related to the exposure (a consistent 6), as well as feared consequences (i.e. the doorknob was not clean, but no specific negative outcome like getting sick).

His parents joined to review Daniel's homework assignment to alternate exposures to the trashcan lid and dishwasher handle at home. To improve adherence and prompt recording of temperature ratings, I introduced Daniel and his parents to Google Sheets, a free, online document application, where we would now be tracking the monitoring. This de-identified spreadsheet allowed Daniel, his parents, and me to keep track of the homework throughout the week. They agreed that it could be helpful for more accurate monitoring and reduce the chance of forgetting the paper record. Finally, I asked Daniel for a final temperature rating, considering he resisted the urge to wash his hands for greater than twenty-five minutes. He reported that since I had last asked for a rating (roughly ten minutes earlier), he had forgotten about the exposure and was not anxious, but once prompted, he rated his anxiety at a three. We briefly explored what he learned from that experienced before the family left.

\section{Phase III: Implementing Graded In-Vivo Exposures and Ritual Prevention (Sessions 6 through 21)}

At this point in the treatment, we began to move away further away from the protocol's plan for each session while maintaining the overall session structure (see Table 10). We did this in order to spend more time on in-vivo exposures and planning of homework exposures, while remaining consistent with the underlying principles of E/RP. As such, Session 6 began with a review of his symptoms, functioning, and homework since the last session.

Specifically, we reviewed the previous in-session exposure of touching the doorknob, and the goal to not wash until get got home, which he reported was unsuccessful. He had been too anxious, and also had wanted to eat something, an activity which required him to wash his hands. In addition, despite the shift to an online format, Daniel continued to struggle with full monitoring, and there were gaps in the homework spreadsheet. After reviewing the homework, and problem solving his difficulty to complete the monitoring, Daniel's parents and I spent the remainder of our time discussing how intertwined families and a child's OCD can become. This component of the manual was moved up to this session (it occurs in protocol as parent-only session 7) based on the assessment of Daniel's symptoms, which in turn provided the opportunity to begin identifying how Daniel's parents accommodate or facilitate his avoidance and escape behaviors. They were helpful in this activity, identifying ways they enable him to avoid perceived contamination. For example, they regularly purchased disposable plastic cups so that he can avoid touching glasses. They understood the rationale behind reducing their involvement in his anxiety, and as a first step, agreed to refrain from purchasing additional cups. 
They also reported that Daniel had picked up dog feces in the house while they were at work. This is a rare occurrence and something that Daniel had consistently refused to do for many months. He reported that in the recent past he would stay upstairs in his bedroom to avoid it, but struggled to identify why he felt able to pick it up this instance, simply saying that he was not as anxious about it. At this point, I began to wonder whether Daniel experienced an excessively sensitive disgust response as a deterrent to picking up dog feces, and what role that might play in his anxiety. However, in the moment, Daniel's success in picking up the dog feces provided the chance to offer praise and encouragement, as well as the repeated rationale as to how and why similar efforts are critical.

After discussing that success, Daniel's parents expressed concerns about the ongoing severity of his symptoms, particularly how much his anxiety interfered with his daily life. They reported that he had not been playing with his friends and that he had avoided getting a haircut, for fear of the barbershop and being touched by the barber. Daniel's parents also wanted to know how treatment could be improved or sped up, given their concerns about the impact of his symptoms. We discussed options, such as increasing session frequency, and I indicated I would follow up after speaking with Dr. Franklin.

With encouragement and repeated prompting for the rationale, Daniel agreed to get a haircut with his father after the session, which would also be included as one of his homework exposures. We also began explicitly discussing ritual prevention monitoring, or the tracking of how successful Daniel was at resisting the urge to ritualize. We framed the effort to "boss back the OCD" as a binary "Daniel Wins" or "OCD Wins," for when successfully resists or he gives into rituals. Daniel and his parents agreed to begin tracking this in addition to the exposures and his anxiety level. Although we did not have time to complete any in-vivo exposures, the parental involvement and the introduction of Ritual Prevention represented time well spent due to their integral role in the success of treatment.

Session 7 (March 7) also began with a review of the exposure and ritual prevention homework, as well as a few minutes problem solving Daniel's ongoing struggles with monitoring completely (he missed one day). As a strategy to prevent this issue in the future, Daniel agreed to set an alarm on his phone to alert him twice a day to fill out the form. Since the last session, Daniel alternated between a daily exposure of touching the dishwasher or trashcan handle (ten seconds each hand, followed by at least fifteen minutes of ritual prevention/resistance). $\mathrm{He}$ successfully completed the homework on only five of seven days for this week, although he exceeded the fifteen minute goal by waiting an extra five or ten minutes before washing.

In terms of this first explicit attempt to track his rate of ritual prevention, Daniel's average rate of Ritual Prevention throughout the week was approximately 8.3\%, ranging from his lowest day at $5.8 \%$ to his highest at $13.3 \%$ successful resistance (see March 7, Figure 5). For total number of rituals completed, Daniel ranged from 13 completed rituals per day to 16 per day (see Figure 3), with one or two successful instances of Ritual Prevention (see Figure 4). Although estimated, the information did give us a baseline to compare to future progress. It also reflected that at this point in treatment he really struggled with resisting the urge to ritualize. It is important to note that we maintained a positive and encouraging attitude throughout, with an emphasis on 
Pragmatic Case Studies in Psychotherapy, http://pcsp.libraries.rutgers.edu

Volume 15, Module 1, Article 1, pp. 1-74, 03-22-19 [copyright by author]

the small successes representing important stepping stones for an eventual reduction in anxiety.

Finally, Daniel and I spent the remainder of the session completing another rating of the CY-BOCS measure to reassess his OCD symptoms. For the symptom checklist, Daniel's obsessions and compulsions remained stable, without any changes. In terms of the severity scales, Daniel's Obsession and Compulsion severity scores were both rated at a 13, with his total severity score of 26 suggesting his symptoms had remained stable, and within the "Severe" range since session 2, approximately five weeks earlier (see Table 1). For homework, Daniel wanted exposures focusing on the television remote control. With prompting for specifics, he agreed to touch the remote with each hand for 20 seconds, followed by contaminating his arms/neck and at least 30 minutes of resistance prior to hand washing. This week's homework represented an appropriate increase in difficulty and resistance time, and Daniel expressed some doubt as to whether he felt he could be $100 \%$ successful.

Prior to our next scheduled session, Daniel's parents contacted me to report that he would be unable to attend due to coming down with the flu. Rather than cancelling, we rescheduled for a phone session the following day, with the aim of a full 45 minutes focusing on resistance, ritual prevention, how to challenge himself more, and how being sick impacts his anxiety symptoms.

However, at the time of the call, Daniel and his parents were driving to his pediatrician due to the severity of his flu symptoms, including fever and vomiting, and so the call shifted to a brief check-in and homework discussion. Despite the loss of an hour of face-to-face session time, Daniel's flu represented an important moment in the overall arc of treatment because it offered a naturally occurring, organic in-vivo exposure targeting some of his greatest concerns: he will get sick, including vomiting, and the suffering will be terrible, possibly too much to cope with.

Daniel and his parents presented for Session 8 (March 21) with a lot to discuss considering his illness and the two weeks lapse since the previous session. Together with his parents, we reviewed his symptoms, including a high fever, vomiting, cold sweats, and fatigue, as well as how being sick impacted his anxiety and OCD symptoms. Daniel highlighted his high discomfort and distress while ill. However, he reported feeling better, and that he had stopped doing his rituals while sick saying there was "no point" once he was already sick. This led to fewer escape and avoidance behaviors related to possibly contaminated aspects of his family and home. When asked about the week leading up to catching the flu, Daniel confirmed that he had consistently ritualized, and tried hard to avoid his father, who was first in the family to fall ill. This was a perfect opportunity to help Daniel challenge his beliefs and perceptions about the role his worries and the compulsions play in his life. Specifically, I posed a number of important questions to target the heart of his anxiety: If the rituals are designed to protect him from becoming sick, and they are not effective at doing so, would he choose to keep doing them? Ultimately, how much control do we actually have over becoming sick? Also, was being sick as bad or as unbearable as he feared or expected?

These thoughts had not occurred to Daniel, but he quickly seemed to grasp the perspective I was hoping he would see: despite his feeling that it was critical to do the compulsion to prevent the feared consequence (e.g. becoming unbearably ill), these rituals are imperfect and ultimately 
cannot prevent that feared outcome from occurring. In fact, although Daniel had not done any homework while ill, we closely examined the monitoring sheet for the week leading up to catching the flu, which showed that he ritualized at roughly the same frequency as other weeks when he did not get ill. Specifically, Daniel successfully resisted the urge to wash his hands approximately $7.5 \%$ of the time, completing seven rituals per day on average and successfully resisting that urge once or none per day (see Figures 4 and 5). Compared to the previous week's monitoring record, Daniel had completed fewer rituals overall (average/day 7 vs. 13.2), but he continued to have significant difficulty resisting the urge to ritualize (7.5\% vs. $8.3 \%$; see Figure 3 and Figure 5, respectively). Daniel's conclusion, that maybe the rituals are not really as effective as he feels they are, was important as a tool to undermine his "reasons" for completing the rituals.

This important insight did not suddenly rid Daniel of his anxiety or the compulsive urge. However, it tied in well to one of the planned session tasks: to try to increase his motivation for exposures, in particular increasing their difficulty. Drawing on his recent experience and newfound conclusions, Daniel was able to complete significantly more difficult in-vivo exposures in this session, taking a big step up the hierarchy compared to past exposures. In fact, together we intentionally and extensively exposed ourselves to various commonly touched objects in the large, busy men's restroom in the clinic, including door handles, sinks, faucets, counters, toilet stall walls/fixtures, metal urinal fixtures, etc. He had previously refused to complete restroom exposures, saying he was not ready, but was now able to push through his anticipatory distress, due at least in part to the knowledge he gained from having the flu. Despite his ongoing worries, Daniel seemed to gain some confidence or motivation after his illness.

After the bathroom exposures, I tapped into this increased motivation during our homework discussion, pointing to his successes as proof he could do more difficult exposures and more frequently resist the compulsion. For homework, Daniel agreed to wait at least 40 minutes before washing, after exposing himself to the remote control and then "spreading" the contamination on his arms, neck, and other exposed skin. For the first time, we also identified a specific ritual prevention goal, meaning he would successfully resist the urge to complete a compulsion at least $30 \%$ of the time.

For the remainder of the time, I met with his parents alone to review the past few weeks and the progress seen with the bathroom exposures. Although the treatment protocol calls for a full parent-only session at this point, the context of the session and Daniel's illness suggested the need for flexibility. Of course, it also helped that we could meet for over 90 minutes, allowing time to cover the content of more than more session. They were excited and a little surprised to hear that he had been willing to do the more difficult bathroom exposure, and wanted to reinforce his learned insights. They described the difficulty of being ill, suggesting low distress tolerance and his tendency to catastrophize about his symptoms before and during any illness. They also expressed that the "cyber" school could be a way that his life had been shifted to accommodate his anxiety, allowing him to avoid situations that would naturally provoke anxiety.

Next, we brainstormed how to further reduce their involvement in the future, with Daniel's mother describing some guilt, and her concerns about what could be contributing to his anxiety. I worked to normalize her experience and reduce any efforts to lay blame. Many parents are unsure 
of how to best respond to their child's anxiety, and are often naturally drawn to soothing and/or accommodating the worries. However, I also encouraged pushing Daniel out of his comfort zone, suggesting it might also take her out of her comfort zone.

Finally, the discussion returned to how treatment could be optimized, and Daniel's parents re-affirmed their willingness to make whatever adjustments might be helpful. I had discussed with Dr. Franklin the question of session frequency during our weekly supervisions, and together we agreed that increasing session frequency to twice a week would be the most appropriate way to increase his treatment "dose." The parents were on-board, but indicated work could make it hard to bring him twice each week, which led to the solution of one in-person session and one remote video session as necessary. Prior to wrapping up, Daniel re-joined in order to get his buy-in for increased sessions. He reported no reservations about meeting twice weekly, but we gave them time to discuss it further before the next session.

For Session 9 (March 28), Daniel and his parents came in a positive mood, as Daniel had gone into four different stores in one day. Daniel and I had repeatedly discussed how Daniel had avoided entering stores or restaurants for the past few years. We also reviewed the rationale for $\mathrm{E} / \mathrm{RP}$, and how a successful treatment would include multiple direct exposures to these environments. With those conversations in mind, it was very exciting to see that Daniel had again jumped up the hierarchy, especially given that these were not assigned or planned. The spontaneity also represented a positive sign, given his past refusal, the significant increase in difficulty, and the fact that his parents successfully encouraged him to do this.

Daniel's monitoring record for that week also reflected some progress, particularly in his rates of ritual prevention, which jumped from the past week's average/day of $7.5 \%$ up to a new high of $23 \%$ (see Figure 5). Daniel successfully reached the $30 \%$ ritual prevention (RP) goal on two of the five days tracked. (see Figure 5). Interestingly, it appeared that the average number of rituals completed per day stayed roughly stable compared to the most recent week (7.5 vs 7), despite the increase in instances of successful ritual prevention (see Figures 3 and 4). When asked to describe his perceptions of this progress, Daniel could not identify why he was able to resist the compulsions more effectively than in past weeks, but it seemed likely that multiple factors contributed, including the minor tweaks discussed in Session 8.

First, choosing a specific ritual prevention percentage goal for each day (30\%) made the task more concrete and obtainable, lowering the bar from resisting all the time to only needing to resist part of the time. Second, adjustments to Daniel's rewards more explicitly and directly connected concrete positive reinforcement to consistent homework completion. Daniel would earn points for each exposure, as well as for reaching his daily ritual prevention goal, rather than rewards doled out by his parents at their discretion. In fact, his mother had reported that his willingness to enter the four stores was primarily related to his expectation of getting a new videogame. Third, Daniel's mother reported that he had followed through on a topic of discussion by posting a note above the kitchen sink to remind himself to resist the urge to wash his hands. These changes, in combination with his increasing confidence, seemed to help Daniel push himself further outside of his comfort zone for both exposures and ritual prevention. 
To capitalize on his increasing tolerance to higher levels of anxiety, I encouraged Daniel to choose an in-session exposure that he had yet to accomplish. He was reluctant to volunteer ideas that would be a true step up in difficulty, which was unsurprising given his high anticipatory distress and tendency to avoid. However, with positive encouragement and a review of the E/RP rationale, Daniel finally agreed to "spread" germs to his face after touching contaminated objects in the office. Alone, touching the office floor or walls would not provoke significant anxiety at this point in treatment because he had already completed those exposures. However, touching those surfaces, followed by touching his face, represented a very difficult task, because he felt strongly that he was more likely to get sick if he touched his face. As I had expected, Daniel reported significantly higher anticipatory anxiety compared to anxiety rating ("temp") during and after the act of contaminating his face. We discussed again how this pattern seems to repeat itself, to reinforce that he expects things to be more difficult than they actually turn out to be.

As usual, we ended with planning homework exposures and ritual prevention goals. He kept the ritual prevention goal at 30\%, given he reached his RP goal on only two of five days, but increased the difficulty of the exposures by adding that he must contaminate his face for all of his homework. Finally, we returned to the frequency of sessions, and Daniel again agreed to meet twice a week. However, he preferred remote sessions over video at first because he was less comfortable with his father driving him to the clinic. He felt his father was not as "protective" as his mother, but eventually acknowledged that his father pushes him more than his mother.

Despite being our first video conferencing session, in Session 10 (March 31), we maintained the overall session structure recommended in the manual, beginning with a review of the homework (see Table 6). Over the two-day span between sessions, Daniel appeared to build upon the momentum of the past week's success, reaching his ritual prevention goal of greater than $30 \%$ resistance on each day, as well as incorporating the face contamination component (see Figure 5). Daniel averaged $40 \%$ successful ritual prevention each of the two days, with an average of six completed rituals and four instances of ritual prevention (see Figure 5). He continued to struggle to verbalize why he felt the homework had gotten easier, but whatever Daniel's internal dialogue regarding his symptoms, he had become observably less distressed and avoidant. I took the opportunity to reinforce that his repeated efforts to confront and "test" his fears were key to his progress and that he was learning valuable life-long skills. I also reminded him of his progress toward another videogame for every day that he reached his goal.

Next, we turned to a brief discussion of his eating habits, as his mother had relayed concerns about possible avoidance of certain foods. To rule out whether he had OC symptoms related to food, I asked him to describe what and how he eats each day, how long he takes to eat, what foods are "contaminated," whether he worries about eating, and whether he avoids any foods. Daniel disagreed with his mother, saying that he was eating normally. However, he did confirm that he frequently takes a long time to meals, and that sometimes he doesn't want to eat too much. When asked to elaborate, Daniel expressed a strong dislike for the "feeling of being full," and a strong desire to avoid that sensation. Furthermore, that "feeling of being full" was closely linked to a feeling that he would vomit, almost as intolerable as touching contaminated surfaces or objects. One significant difference from his worry about germs, however, was that Daniel denied any compulsive behaviors associated with his worry about feeling full or vomiting, suggesting that these 
Pragmatic Case Studies in Psychotherapy, http://pcsp.libraries.rutgers.edu

Volume 15, Module 1, Article 1, pp. 1-74, 03-22-19 [copyright by author]

concerns fall under the secondary diagnosis of Generalized Anxiety Disorder. Daniel had not endorsed these worries during Phase I (Assessment), although it is common to uncover other dimensions and details regarding a child's anxiety during the process of exposure.

As a result, we discussed the concept of disgust. I detailed how people can become hypersensitive to their internal physiological sensations, such as nausea or stomach aches, as well as how anxiety can contribute to or exacerbate those sensations. This can lead to a selfreinforcing cycle of hypersensitivity and somatic symptoms, and consistent with anxiety, avoidance of those sensations leads to greater worry about them. As another opportunity to reinforce the E/RP model and rationale, I highlighted that his worry about feeling full and throwing up were similar to OCD because he can also easily engage in exposures to "boss back" those worries. Continuing in the assessment mindset, Daniel walked around the house with the laptop, showing the home and identifying areas and items he perceived as highly contaminated.

We utilized the remainder of this hour-long video session for in-vivo exposures. Daniel exposed his hands to a dirty and dusty cabinet full of his games, followed by "spreading" the contamination to his bare arms, neck, and face. In addition, Daniel ate applesauce with a spoon that he deliberately contaminated on the kitchen counter. In both of these exposures, Daniel rated his temperature as a 7, while successfully refraining from ritualizing for 50 minutes. Daniel found it easier to do more challenging exposures like these in my presence, such as eating with the contaminated spoon, and even my virtual presence facilitated an exposure that he had avoided completing on his own as homework. Thus, this first video session reflected some the strengths of using video technology in OCD treatment, as we were able to provoke greater levels of distress in a more relevant context than the office. Location and context can be critical components of an individual's obsessions and compulsions, and therefore, it could be doubly important for generalization of learning to do as many exposures in as many of the locations and contexts as possible.

For Session 11 (April 4), Daniel and his parents again presented in a positive mood, based on ongoing progress in increasing exposure difficulty and frequency of ritual prevention. Our review of his homework showed Daniel had reached the $30 \%$ ritual prevention goal on each of the four days since the last session, a first for him. In fact, he exceeded that goal, successfully resisting the compulsion at least $40 \%$ of the time (daily average of $41 \%$ ), while averaging 6 rituals per day and 4.16 instances of ritual prevention (see Figures 5, 3, and 4, respectively). In addition, Daniel noted in the "Comments" column that he had eaten food after dropping it on the couch, another thing he certainly avoided in the past. I repeatedly praised and encouraged Daniel to maintain this level of effort and commitment to completing the homework, reinforcing the critical importance of working to reduce his avoidance and directly expose himself to his fears.

I prompted Daniel to try to identify how his motivation may have changed, considering his increased willingness for more difficult and novel exposures. He again had a general feeling that it had gotten easier, while also expressing motivation for getting a new videogame. We also talked about how anxiety impacts his social life, including whether he has any interest in dating and how symptom reduction might impact that part of his life. Daniel demurred, appearing slightly uncomfortable, but did say he would enjoy spending more time with his friends playing 
videogames or going outside. During my time alone with Daniel's parents in the last session, his mother wondered whether he thought about dating, expressing concerns about his healthy development. I shared some of her concerns, and also felt it was a chance to help reinforce or even increase his motivation to stay consistent with the homework and complete more challenging exposures.

Next, we turned to completing additional in-vivo exposures in and around the office. I began giving Daniel a menu of options for possible exposures to complete during the session, as well as for homework, with the aim of gradually increasing the exposures and RP difficulty. The options ranged from slightly more difficult than previous exposures, up to stimuli outside of his comfort zone. For this session, I suggested contamination exposures either in the lobby and right outside of the building, or an exposure to that feeling of being full, which would be done by drinking carbonated seltzer water. Daniel chose to touch various surfaces in the lobby and outside the building, although he was unwilling to contaminate his face. Even without that additional component, Daniel's willingness and successful exposure to those contaminated surfaces represented another significant step forward. Only a few weeks prior, he felt these specific exposures were overwhelming, but during this session, he touched stimuli including walls, pillars, metal railings, glass doors, and an outside ATM, all without significant visible distress.

After the exposures, we reviewed his experience back in the office, emphasizing the important parts of the task, and generalizing his learning as much as possible (i.e. "If you can do this, can you do that?') Daniel's parents joined for the remainder of the session and helped to provide praise and encouragement. Their presence also allowed for a transparent discussion of their view of Daniel's progress, and their own efforts to reduce accommodation of his avoidance. They had only positive remarks about his continued progress, while also noting how he remains anxious. They reported some challenge in identifying and resisting their past habits of accommodation in the moment, such as opening doors for Daniel (removing the natural exposure to the door knob). Despite their lack of confidence, I continued to reassure them that habits take time to change, and to remember why their efforts are important to Daniel's future.

Next, we planned his homework, and with his parent's encouragement, Daniel said he would try to go to a store or restaurant before the next session. I also pushed him to try these high-level exposures given his recent successes, and also suggested that he reach for a goal of $50 \%$ ritual prevention, to which he agreed. Daniel did not sound particularly confident or excited about the exposures, but it was important to get him back to doing common tasks similar to these. As the session wrapped up, and as a reward for his in-session and homework efforts, Daniel showed me a mobile videogame for the final five minutes.

For Session 12 (April 7), we returned to video conferencing technology for a remote session. Our review of the homework revealed more difficulty with ritual prevention over the two days between sessions, with an average of 6 complete rituals, as well as a drop to an average of 3.5 instances of successful ritual prevention (see Figure $3 \& 4$, respectively). In terms of the percentages, Daniel fell short of the $50 \%$ goal both days (41\% and $28 \%$; see Figure 5). He did not have a clear insight into this slight step back compared to the very successful week prior, but we spent a few additional minutes problem-solving strategies to help boost his efforts. That being 
said, he did increase the homework exposure difficulty, specifically by exposing himself to new stimuli: doors and walls inside and the deck outside the home. Daniel also independently completed two unplanned, but high-level, exposures: cleaning up dog feces in the backyard and playing outside with his friends for an hour. I reinforced Daniel's choice to push outside of his comfort zone for homework and reminded him of the rewards, especially because he was earning rewards for things he enjoys.

In-vivo exposures to novel stimuli in his home followed that discussion, and I again provided Daniel with a menu of options. Specifically, with me coaching via video, Daniel exposed his hands, arms, neck, and face to the following things in and around his home: exterior shingles, basement walls, and the basement floor. He also again ate food with a "contaminated" spoon, after not checking if it was clean and deliberately getting germs on it by putting it onto the counter. It was important to continue to expose Daniel to new and more difficult things as often as possible, due to the importance of generalizing Daniel's exposures, and thus his learning, to as many context-relevant stimuli as possible as quickly as he is willing.

Finally, Daniel chose exposures for homework for the next session. I reminded him he had successfully avoided doing a restaurant or store exposure during the previous week, and encouraged him to do at least one before the next session. We created a more detailed plan for how those exposures would look, as it seemed that he may have been overwhelmed by anticipatory anxiety because a lack of specific forehand knowledge led him to assume the worst. We honed in on very specifically which store or restaurant he would go to, how long he would be there, and how he might feel. In addition, he wanted to keep the 50\% daily ritual prevention goal, expressing confidence that he could achieve that. This greater specificity seemed to bolster Daniel's confidence, or at least willingness, but he also identified other, house-related exposures he could do as alternatives. I prompted him to apply the rationale and model for E/RP to his specific symptom, in order to reinforce the importance of doing things he feels he wants to avoid. Daniel provided good answers that were consistent with our discussions, and it appeared to help ground his expectations for the exposure homework.

Session 13 (April 14) fell a week later as an in-person session at the office, and our homework review again pointed to a sometimes common theme in pediatric OCD treatment: effort and progress can vary significantly depending upon the client and what may be going on in their lives. Moreover, individual clients vary significantly in terms of their attitude, effort, and progress on the two main components of treatment, exposure and ritual prevention. This variability can unfortunately sometimes lead to a "one step back for every two steps forward" feeling. Daniel's monitoring over the preceding week showed that he had done well for the first four days, but struggled for the remaining three days.

In terms of those first four days, Daniel and his parents stayed at the beach in their $\mathrm{RV} /$ camper for the weekend, an anxiety-provoking situation on its own. He was rewarded for multiple aspects of this trip, as he engaged with naturally occurring stimuli and situations that he had been avoidant of in the past, including the two-hour car ride and sleeping in the camper overnight. For ritual prevention, he had actually reached the increased goal of $50 \%$ successful ritual prevention on three of the four days tracked, with an average ritual prevention rate of 
Pragmatic Case Studies in Psychotherapy, http://pcsp.libraries.rutgers.edu

Volume 15, Module 1, Article 1, pp. 1-74, 03-22-19 [copyright by author]

$45.75 \%$ for the week (see Figure 5). The total number of completed rituals fell to 4.75 per day, while instances of successful ritual prevention came in at an average of 4 per day (see Figures and 4 , respectively).

The weekend was not without some challenges, however, as Daniel indicated he stayed inside due to bad weather and also had not completed any exposures. Asked to explain his reasoning for not doing any exposures, Daniel reported that he although he had was not entirely sure, but it seemed he simply preferred not to do the exposures and that the bad weather felt overwhelming. At this point, I asked him to again identify what the E/RP model and rationale would say about that type of choice. Although Daniel reported that he had completed exposures each of the last three days, he had not recorded details about those exposures nor did he keep track of his ritual prevention. Unfortunately, I had to remind him that he would not earn rewards because he did not keep track, while encouraging him to keep up his efforts all the same. Given his seemingly variable motivation, I expressed my continued confidence in his ability to complete all exposures and reach his daily RP goal. However, I tried to be balanced, by providing praise for the four days that he did well, while sharing my opinion that he could do better, considering his progress and success over the past few weeks. Next, Daniel and I planned homework exposures given that the family would be returning to the beach. We brainstormed a variety of options for Daniel's "exposure menu," and he expressed a willingness to try going to a store prior to the next session. He also agreed again to increase his ritual prevention goal, this time from $50 \%$ to $60 \%$.

Finally, we completed another rating of the CY-BOCS measure. The severity of Daniel's obsessive and compulsive symptoms had decreased compared to the last rating, and in fact, had fallen out of the "Severe" range and into the "Moderate range." Daniel had obsessive symptom severity of 10 (compared to a previous 13), a compulsive symptom severity of 7 (compared to a previous 13), and a total severity score of 17 (compared to a previous 26; see Table 1). These numbers reflect Daniel's progress with E/RP tasks, and that his efforts were paying off despite any incremental ups and downs. These moments are critical opportunities to bolster confidence and reinforce what was making the difference: consistent exposure and ritual prevention.

Our next clinical contact was again in the office, and Session 14 also began with a checkin and homework review. Daniel wrote the following for the exposures he completed during their second weekend at the beach: Day 1) "went to the shore with no issues" [temp=0]; Day 2) "used the same glass almost all day" [temp=0]; and Day 3) "1. Went to one store [temp=4], 2. Tried to do homework outside but worried about bees so went back in [temp=8]." As evidenced by the two zero "temp" ratings for Day $1 \& 2$, these were not sufficiently challenging tasks, and it seemed he had fallen back into his comfort zone. Some questions targeted at his specific thoughts, feelings, and symptoms about the beach and the RV revealed that Daniel experiences a moderate level of anxiety simply traveling to the beach and staying overnight in the RV. He was anxious about the contamination of the vehicle itself, and was also significantly anxious simply being outside at the campground, at the beach, and in particular, at the beach boardwalk (which he had consistently avoided). He described the campground as dusty and dirty, while the beach and boardwalk were too busy with probably contaminated people. 
With additional Socratic questioning, Daniel communicated his experience of elevated baseline anxiety during these beach weekends, and how it added to his avoidance of challenging homework exposures for the first two days. However, ultimately, Daniel did another store exposure on the final day, specifically a few minutes in a grocery store with his mother. It is important to note how his parents' ongoing encouragement and "pushing" have helped him overcome his established patterns of anticipatory anxiety and avoidance. Interestingly, once started, Daniel rated his anxiety level at a 4 , and which was very manageable in my mind, based on what he had done so far (i.e. more anxiety provoking things that that).

With that praise still hanging in the air, we transitioned to in-vivo exposures, specifically to a food related exposure that I had discussed with his parents. After agreeing upon specifics, Daniel and I exposed ourselves to the walls and floors in my office, before eating a lunch of chips and sandwiches. He expressed some trepidation at how difficult this would be, but he successfully ate with his bare hands, and did not have to wash. In fact, Daniel again experienced greater anticipatory anxiety prior (temp=8), compared to during (temp=6), evidence that it was not as bad as he expected. Daniel and I spent the remainder of the session discussing homework, and negotiating what would be included in his next "Exposure Menu." He agreed to the following options: 1) go to store, contaminate self while there, 2) contaminate hands, then touch food and eat, and 3) contaminate silverware with hands then eat with it.

Overall, a pattern had begun to emerge in the treatment, such that Daniel would make good strides with exposures and ritual prevention for a few days, before struggling to fully monitor his homework or avoiding more difficult tasks. I returned to considering his motivation and decided to be more involved in tracking the points he earned for each exposure, as I realized that he was not able to visualize easily how much he had earned or would earn. Clearly seeing the rewards would probably not be a "silver bullet" for his up and down effort, but it seemed to help to some degree over time, because he had more specific detailed information about exactly how his homework led to new videogames.

Session 15 (April 21) was another remote video session, which provided the opportunity to focus on things he had struggled to do independently in the past. However, prior to jumping in, we again reviewed the homework since the last session. Over four possible days, Daniel again had only partial completion of the homework record, specifically tracking exposures on all days, but only tracking two days of ritual prevention. He continued to say he forgot to track the information, and I again reminded him of the importance of accurate monitoring to the treatment outcome.

Unfortunately, Daniel did not reach the $60 \%$ ritual prevention goal on any of the days, which suggested more resistance. (see Figure 5). His average completed compulsions per day rose from 6 to 7, while his "wins" or successful ritual prevention fell from an average of 5.5 per day to 4.5 per day (47.5\% average/day vs. $39 \%$ average/day; see Figures 3 and 4, respectively).

However, Daniel's ongoing struggle with ritual prevention was balanced by some good progress with the difficulty of his completed exposures. He recorded that he completed the following tasks for homework: 1) mowed the lawn 2) "touched the family room walls \& rubbed 
my spoon on the island before eating my cereal," 3) "went into Kmart," and 4) "went into Chick Fil-A." He received significant praise and encouragement for all of the exposures, with an emphasis on going into the stores. He reported distress while mowing the lawn and entering the stores, but had not given into the urge to avoid or escape. In addition, Daniel again experienced greater anticipatory anxiety compared to during the exposures. Although he had not eaten inside of Chick Fil-A, entering the building represented a challenge for him, and therefore a positive step forward, while also pointing toward an important future exposure.

Transitioning to in-session exposures, Daniel built upon his progress by contaminating himself by touching the outside deck and fireplace without washing. Next, he immediately handled and ate multiple grapes with his bare hands, again without washing, rating his "temp" as a 7. Daniel completed a second exposure after briefly discussing the first, in which he spread germs to the inside and outside of a glass with his still contaminated hands. He then drank water from the glass, and again rated his anxiety at a 7. Interestingly, throughout both of these exposures, Daniel did not show any reluctance to engage, and appeared less visibly distressed compared to earlier in treatment, which suggests improvements in his distress tolerance. Thus, despite his struggles with inconsistent RP and RP monitoring, Daniel continued to make gradual progress with increasing the difficulty of completed exposures.

Daniel and I ended our session after agreeing upon homework exposures and ritual prevention goal for the next session. Using his success of entering Kmart and Chick Fil-A, I pushed him to do more food and store/restaurant exposures. These tasks were at the top of the hierarchy or "fear ladder" created at the beginning of treatment and show his significant progress. He was still anxious and somewhat avoidant of the higher-level exposures, but that anxiety had dropped to the extent that he could begin to engage more regularly with stimuli or environments that he had previous avoided entirely. Specifically, he agreed to the following options: 1) "eat in restaurant w/o washing hands," 2) "Go to store, touching lots of stuff w/o washing," 3) "Get hands dirty by touching things, and then eat w/ fingers," 4) "Get fork/spoon/cup dirty w/ hands and then use it." Finally, Daniel kept his daily ritual prevention goal at 60\%, because he felt confident despite my offer to lower it, and I praised him for continuing to challenge himself.

For Session 16 (May 2), I met with Daniel and his parents at the office, shifting the protocol's standard session structure to include the entire family to complete some further assessment. First, as usual, we reviewed his homework and provided encouragement to continue his efforts. Based on the record, Daniel completed roughly eight exposures over the ten days between sessions, although the difficulty of the specific tasks varied significantly. On four of those days, Daniel did sufficiently hard exposures, or those that represented a greater challenge, rather than a repeated one. Specifically, these were: 1) went to a grocery store, touched items, ate lifesavers without washing, 2) went to the beach for an hour with parents, and 3) went into Dunkin Donuts. On the other days, Daniel either repeated a past exposure (so not as difficult) or did not record any exposures. He reported again that he was not motivated to do exposures on those days.

The exposure progress contrasts with the continued variability in ritual prevention, as he again struggled to achieve the daily ritual prevention goal of $60 \%$, in this instance reaching it on 
only one day. However, the other metrics reflected some improvement compared to the previous week. He had completed on average 5.83 compulsions per day, with 5.5 "wins" or instances of successful resistance, which is a rate of $48.5 \%$ for daily average ritual prevention (see Figures 3 , 4 , and 5, respectively).

As previously mentioned, Daniel's homework reflected inconsistency in regular completion of exposures and RP, accurate monitoring of those tasks, and a steady increase in the difficulty of exposures. For the past few weeks, Daniel and I had discussed his progress, and how we were getting closer to the top of the hierarchy. His avoidance of the higher-level exposures (e.g. going into a store or restaurant) had reduced to an extent, as evidenced by his increasing ability to engage with those stimuli, although he wanted to avoid the most difficult exposures.

Thus, we spent considerable time brainstorming novel stimuli and situations that he still needed to engage with, focusing on trying to answer two questions: What things does Daniel still try to avoid? When/where does Daniel still feel the urge to ritualize?

This yielded a variety of new possible exposures, and also led Daniel to ask his parents numerous reassurance seeking questions related to health and cleanliness. For example, his mother noted in the Comments column of the homework spreadsheet that he "asked a lot of questions before going [to the beach]: Am I going to get dehydrated? Get a stroke? Get sick?" After the family described the questions, I provided psychoeducation about reassurance questions and how they also fall into the negative reinforcement cycle of avoidance that functions to exacerbate his anxiety. I tried to normalize their experience, stating that it is a natural reaction for a parent to try to reduce their child's distress, even if it inadvertently reinforces those symptoms in the long term.

Daniel was unsure whether he felt those questions were actually rituals, but did agree that he felt significant relief when his parents answered. This prompted us to brainstorm strategies to reduce the parents' side of the equation, including not answering his questions or providing incorrect or incomplete answers. He was not happy about the possibility of his parents pulling back in this way, but we reminded him of his recent successes, especially emphasizing his ability to tolerate distress. We also discussed the challenge in reducing the total number of compulsions, tying this difficulty to the E/RP rationale and model about why it is imperative to reduce that number. For example, in the past week, Daniel had washed his hands (or otherwise ritualized) anywhere from four to eight times a day. He and his parents were unsure of a "normal" amount of hand washing, but he ultimately wanted to follow a hard rule of only washing his hands prior to eating, so three times per day, as long as the washing was not in response to worry, distress, or an urge to ritualize. If it did become a ritual, he was instructed to immediately re-expose himself.

Next, Daniel chose the following "exposure menu": 1) Do laundry 2) Load dishwasher 3) Sit in restaurant while picking up takeout 4) Touch trees in backyard, and 5) Touch dirty game cabinet. The daily ritual prevention goal remained at $60 \%$, based on his ongoing difficulty to reach it. In addition, his mother requested that they go to a movie together, and while Daniel was not willing to agree to it outright, he indicated he would try because it was Mother's Day. 
Finally, I spent a few minutes alone with his parents to return to the topic of next year's school plan. I expressed my concern about keeping Daniel in an online program, within the context of the treatment's focus on reducing avoidance and increasing engagement with his worries. His parents identified Daniel's attendance at a brick and mortar school as representing a future goal, but not what they planned for 9 th grade. They felt he had been making academic gains in his online program and that he learned more in this format compared to his prior school, at least in part due to his anxiety. I empathized with their feeling about his improved academic performance, while repeating the E/RP model and our overarching goal to engage directly with what can be hard or scary. They acknowledged the ongoing avoidance, and I encouraged them to get him back into weekly activities or summer camps, to have at least some of the social interactions, and natural exposures, that would occur in a school setting.

For the first portion of Session 17 (May 5), another remote video session, we reviewed the three days of homework. He did the following exposures on two of the three days between sessions, with one day of no exposures: 1) sat in Subway sandwiches for 5 mins, 2) used only one glass to drink, 3) touching cabinet. Although still a positive effort overall, sitting in Subway for 5 minutes while his mother ordered a sandwich, as well as the other exposures he completed, were not as difficult as I felt he could tolerate at this point. For ritual prevention, Daniel successfully followed the new "no more than three hand washes per day" rule, on two of three days, which led to daily averages of 4 completed compulsions, 4 successful ritual preventions, and a 50\% successful resistance rate (see Figures 3, 4 and 5, respectively). Another good step, considering those were Daniel's lowest average rituals per day, and his highest rate of successful resistance, up to this point in treatment.

After much praise and a detailed review of what contributed to the progress, Daniel and I utilized the remainder of our time for assessment. We returned to identifying novel and/or more difficult exposures, which we had begun with his parents in the last session. In the course of this activity, Daniel revealed additional specific and useful details about his anxiety that he had not previously verbalized. Daniel reported his worries increase as the number of people around him increases, and that his worries about an increase in contamination often contributed to avoidance. $\mathrm{He}$ also reported that he worries about being anxious in those situations, to such an extent that he worries he will vomit. Thus, Daniel believes the longer he spends somewhere, and the more people there are, the more likely he is to become nauseous and throw up. This has never occurred, and Daniel has never vomited in public, or even come close. He can recognize that these worries are not rational with me in session, while also responding to his distress and falling back into his established patterns of avoidance.

Finally, prior to ending the session, Daniel agreed to continue with the same "Exposure Menu" as the previous session due to the short duration between sessions. I also encouraged him to attend a movie with his mother, as an important task in therapy and an important gift for Mother's Day. He again expressed a mixture of reluctance and willingness.

Daniel and his parents were happy when they arrived at the office for Session 18, as he had successfully attended the movie. In fact, he spent upwards of two hours at his older sister's house prior to the movie, a place he had consistently avoided. Daniel described a crowded movie 
Pragmatic Case Studies in Psychotherapy, http://pcsp.libraries.rutgers.edu

Volume 15, Module 1, Article 1, pp. 1-74, 03-22-19 [copyright by author]

theater, as well as his anticipatory worries about getting nauseous in the theater. He seemed to be concerned not only with becoming ill, but also having a lot of people watching him throw up.

However, he reported not feeling sick at any point in the movie, and it appeared to be quite a positive corrective experience. His body language and overall demeanor suggested he was proud of himself. In addition to the movie, Daniel also made great progress by completing a few other anxiety-provoking tasks without washing or otherwise ritualizing, specifically: 1) "Sat at [Dairy Queen] for an hour. Was mad at first but got better. I ate french fries \& ice cream there but ate my chicken at home"; 2) Load the dishwasher (from Mom: "Daniel held his nose while emptying the dishwasher"); 3) He went into Gamestop to look at video games.

I reflected back to him that his anticipatory anxiety seems to increase his avoidance, but that once he decides to do something, he has very little difficulty in completing the task. Over the course of treatment, Daniel proved his initial perceptions wrong over and over via the exposures, but only gradually was able to change his avoidance habits. The past few weeks of treatment had seen additional progress in terms of Daniel completing more difficult exposures. There also had been significant progress with his ritual prevention efforts, as he had fully integrated his new rule of only washing his hands prior to meals. In fact, he only washed his hands 2.33 times per day on average, his lowest so far in treatment, while successfully preventing the urge to ritualize $53 \%$ of the time (average of 2.66 "wins against the OCD" per day; see Figures 3, 5 and 4, respectively). Daniel's implementation of that new RP shorthand for himself seemed to help him to resist the urge. Despite Daniel beginning to wash his hands at a more developmentally typical rate (i.e., 2-3 times/day), he still experienced distressing intrusive thoughts and the urge to wash his hands in order to cope with those thoughts. Understandably, then, he had more exposures to go.

For the rest of the session, we worked to reinforce learning he acquired through his exposures this week (e.g. he can go to the movies!), as well as to generalize that learning to other situations (e.g. he can also eat a full meal at a restaurant). This included repeated Socratic questioning to gauge his knowledge of OCD and E/RP.

For the past few weeks in supervision, Dr. Franklin and I had discussed Daniel's variability in his willingness to increasing consistently the challenge of his exposures. We conceptualized this as variable motivation, exacerbated by his strongly established avoidance habits. However, during this session's debrief of his exposure progress, it seemed that Daniel also had significant difficulty verbalizing his knowledge of OCD and the E/RP model and rationale. He had difficulty clearly defining obsessions and compulsions and was vague when describing what he should do in a hypothetical situation. We could not clearly account for how Daniel had gotten to this point in treatment without this knowledge. We talked about these topics repeatedly during sessions, and I always made an effort to verbally confirm with him that he understood. However, whether he simply struggled to express his thoughts in that moment, or I failed in my delivery of psychoeducation, Daniel's lack of clarity on some really important concepts and definitions suggested the need to reintroduce reinforce his knowledge. It also represented an opportunity to repeat the overarching idea that he needs to stop avoiding what he worries about to get over his anxiety, and in fact, he should repeatedly engage with that which concerned him the most. 
Back in the office for Session 19 (May 16), Daniel and his father reported further progress, although this week also followed the non-linear pattern that was becoming clearer over time. The family had again spent the weekend at the beach, and as his most difficult exposure that week, Daniel had walked around the beach boardwalk for upwards of two hours. He expressed how difficult it was (temp=10), because the boardwalk hosts an outdoor market that had attracted a crowd to an already busy beach. Daniel had tolerated two hours of walking around in a crowd of possibly contaminated people, while also touching various stimuli (e.g. clothes on racks, items on shelves). Previously, he had resisted the boardwalk as an exposure, due to how anxietyprovoking it was, and when asked how/why he was able to do this now, Daniel did not have considerably clear insight. However, it seemed that Daniel felt less anticipatory anxiety, as well as greater confidence in his abilities. These internal factors seemed to be enhanced by his parents' efforts to push him to reduce his avoidance, encourage him out of his comfort zone, and by the concrete rewards connected to the homework.

In addition to the boardwalk, Daniel also completed the following exposures that week: 1) haircut by barber; 2) pick up dirty clothes off floor with bare hands; 3) load dishwasher with dirty dishes (x2); and 4) cleaned up dog feces off floor with napkin ("I washed my hands after"). Cleaning up the dog feces off the floor was a difficult task and represented a good effort to reduce his avoidance. That being said, some of those other exposures were not especially high on the hierarchy. For the ritual prevention homework, Daniel only achieve a 50.8\% daily average successful resistance, which was again below the daily goal of $60 \%$ and slightly below the last week (53\%; see Figure 5). His daily average of compulsions remained approximately the same at 2.83, while instances of successful ritual prevention rose slightly to 3 (see Figures 3 and 4, respectively).

This repeated pattern of non-linear progress, and the apparent weaknesses in his knowledge of relevant concepts, prompted me to return to topics introduced early in the manual, to reinforce Daniel's knowledge about E/RP and OCD. Our discussion again reflected that Daniel reported feeling motivated by treatment, while continuing to struggle with delineating between obsessions and compulsions. He also had some difficulty identifying how the model says he should act when confronted with hypothetical anxiety-provoking situations. Where he was less certain, I provided corrective information and pushed him for the right answer. Daniel's struggle to verbalize concepts may not mean he did not possess the knowledge, but it is reasonable that this may have slowed, or otherwise affected, the treatment effectiveness.

Finally, we re-administered the CY-BOCS to gather updated information regarding the severity of his symptoms. Daniel endorsed obsessive symptoms with a severity rating of 9 and compulsive symptoms with a severity level of 9, which led to a total severity score of 18 (see Table 1). Overall, Daniel's symptoms remained roughly the same compared to the rating from a month prior, and again reflected that pattern of non-linear progress. His father and I reminded him that despite the CY-BOCS number remaining stagnant, he continued to make positive steps forward in treatment. Further, Socratic questioning helped Daniel express: the best way to lower that number would be to complete increasingly difficult exposures and continue his efforts to resist the urge to ritualize or avoid his worries. 
In the week leading up to Session 20 (May 23), Daniel continued to push himself. The family walked around the Philadelphia Zoo together for over two hours and Daniel even ate an ice cream. His mother commented in the monitoring form: "Daniel did great at the zoo. No issues." Daniel also did a range of other exposures that week, all of which he had consistently avoided pre-treatment: 1) "Daniel put dirty dishes into dishwasher" 2) "went to Gamestop" 3) picked up dirty clothes w/ hands 4) Went w/ parents to the zoo for two hours 5) Did dirty laundry (two different days); 6) "Played outside with friends for a couple of hours." In addition to the exposures, Daniel washed his hands 4 times each day on average, with an average 3.8 instances of ritual prevention, which led to a daily ritual prevention average of $48.7 \%$ (see Figures 3,4 and 5 , respectively).

From the perspective of his functioning, Daniel, his parents, and I were able to point to how many things he had recently done that he avoided for months or years, such as going to the beach, boardwalk, movies, or the zoo. These represent important steps toward Daniel returning to a developmentally appropriate lifestyle that involves engaging fully in all aspects of daily life. They also get us closer the family's original hope for treatment: that he would be able to participate in activities he had previously enjoyed or discover new ones. We also continued to consider the contradictions in treatment. Specifically, Daniel's ongoing struggle to extinguish the final few compulsions each day (over his 3 per day rule), despite his ability to intellectually acknowledge the importance of doing so, and the gradual increase in the difficulty of his completed exposures.

Consistent with my strategy over the past few sessions, I again prompted Daniel to describe aspects of the E/RP model and rationale using his own experiences and symptoms to strengthen his knowledge and ability to express that knowledge. Repeated rehearsal of important concepts of E/RP and OCD, in the context of hypothetical scenarios he might face, seemed to help him more easily link that information to changing his behaviors. Having his parents present for the entire session provided a secondary, but important, benefit by improving their own knowledge about how to help him apply central concepts to changing his behaviors. We reflected on the importance of their role in the treatment and how Daniel's high anticipatory anxiety prevented him from volunteering to do the hardest exposures. Positive encouragement and "not letting him off the hook," as his father expressed, seemed to be major contributing factors to Daniel's trips to stores, restaurants, and the zoo. I encouraged Daniel, and his parents, to continue to target things that he found difficult or avoided, such as eating a meal at a restaurant.

I also emphasized the importance of continuing to improve upon his ritual prevention, and clarified what it meant to ritualize and what was appropriate hand washing. He was resistant to the idea of an extended period without hand washing, despite my explaining how this could help to extinguish fully those remaining symptoms. However, it seemed he got the message that in order to achieve his goal of getting the OCD under control, he would have to significantly step up his efforts to stay consistent with his own rule of washing his hands strictly before meals.

After discussing his exposure "menu" options for homework, Daniel and I shifted to completing two in-vivo exposures. We had been reviewing what he still found uncomfortable, and identified his socks as something he still had not deliberately exposed himself to. As such, he 
touched his socks and then spread that contamination on his arms, neck, and face. Next, in order to try to really provoke some significant anxiety, Daniel and I left his parents in the office, and walked down a busy Philadelphia block to a nearby 7-11 convenience store. During the walk, we reviewed the purpose and details of the exposure, specifically that we would walk around the store and pick up items to examine them. Once he had touched at least ten different items from as many parts of the store as possible, he would go up to counter and purchase one item. Daniel completed this and the sock exposure with no visible distress, and described his "temp" ratings varying from 2 at the start to 1 or 0 at the end. I reminded him that if he can do these tasks with me, without feeling much anxiety, he could certainly eat a meal at a restaurant or fast food joint.

During the previous session's homework brainstorming, Daniel and I agreed to a challenging in-vivo exposure together to be completed over the phone for Session 21, which we would then debrief using video conferencing. However, our planning fell to the wayside in the face of Daniel's eagerness. Immediately after picking up my call, Daniel reported he just left the store having completed the entire exposure, including an unplanned trip to the bathroom without washing his hands. He returned to his home, we transitioned to video, and began to discuss his experience going into the grocery store alone, including using its public bathroom. Daniel reported that it was "fine, easy actually," and that he had not felt anxious. He seemed to minimize the progress this represented, but I reminded him of his past avoidance to provide context.

Some might disapprove of Daniel's lack of hand washing after using the restroom, but in the context of contamination OCD, not washing his hands was great progress. Daniel had recently described some efforts to coordinate using the restroom prior to meals so that he could wash without adding to his total number of rituals. In that session, I had prompted him to reflect on this choice based on the E/RP model, and he correctly identified he should stop trying to time his hand washing. Using a public restroom without washing was a significant step past that, and I reinforced the importance of what he did.

During the video session, Daniel and I also reviewed the other exposures that he had completed since the past session: 1) did dirty laundry, 2) mowed lawn, 3) "Daniel went to the grocery store with friends to buy stuff," 4) "He also went to the Verizon store," 5) Emptied the dishwasher. Consistent with previous weeks, Daniel completed some challenging exposures as well as some less challenging or at least repeated ones. Unfortunately, Daniel had not recorded his ritual prevention over the week, so we did not have information to compare to his goal. While he had not monitored, he stated he consistently washed his hands no more than three times per day and only before meals. He also reported that when he washed prior to meals, he did not do so because he felt the urge to ritualize or because he was particularly anxious. I asked a number of follow up questions about his thoughts, feelings, and behaviors in these situations, and Daniel consistently expressed he didn't feel he was ritualizing during the past week of only hand washing prior to meals, and that it felt different to him. I took this at face value, while also encouraging him to immediately expose himself (for example by touching the counter or sink) if he felt his behavior was compulsive or was designed to manage his anxiety. Finally, we planned for the upcoming week of exposures, in particular how we can get the most out of their upcoming weekend at the beach. 
Pragmatic Case Studies in Psychotherapy, http://pcsp.libraries.rutgers.edu

Volume 15, Module 1, Article 1, pp. 1-74, 03-22-19 [copyright by author]

\section{Phase IV: Maintenance and Relapse Prevention (Sessions 22, 23, \& 24)}

For Session 22, Daniel, his father, and I met in the office. Daniel's homework sheet again showed him completing some great (and challenging) exposures at the beach and at home, while also showing that he had not been tracking fully (his mother commented that she had actually kept track this week). For the exposures, he did the following, with his mother adding the additional, quoted detail: 1) "Daniel rode in car two hours, no issues," 2) "Daniel survived the beach for 1.5 hours, we stopped in to eat at a pizza place and his stress was 13," 3) Helped build a fire pit with his bare hands, no distress, 4) Went golf cart shopping w/ Dad, 5) Cleaned up a very dirty old bike w/ bare hands (got chain oil on them) 6) played outside w/ bike and scooter, no issues, 7) Went to a store, and 8) emptied dishwasher.

We all highlighted how Daniel had gone to the beach and even ate pizza there, and his willingness to get dusty and dirty while building a fire pit and fixing an old bike. Daniel seemed to "break through" in terms of progress with exposures over the past few weeks, with a large decrease in levels of worry and distress both during exposure and more broadly across the day. Moreover, for the situations that still remained anxiety-provoking, I strongly reinforced the idea he had grown so much given how much he anxiety he could tolerate, such as eating pizza at the boardwalk, which he described as a "temp" of 13 out of 10 . The seemingly precipitous decrease in his distress actually mirrored other periods during the course of treatment, when he would finally stop himself from avoiding a particularly challenging exposure. This would also coincide with drop in his levels of distress. Over and above the specifics, Daniel's father kept coming back to the profound shift they had seen in his behavior, temperament, and attitude about life. He saw Daniel as more involved in his own daily life, significantly less preoccupied with worries, and that he does not "think about stuff as much as before." Overall, his parents saw him as willing to do most developmentally appropriate tasks or activities, inside and outside of the house, which represented such a significant change compared to before treatment.

In response, I asked Daniel and his father to talk about how they thought treatment should go forward, particularly regarding the focus and structure of sessions, in light of how we seemed closer to the family's initial treatment goals of Daniel returning to healthy functioning. To help ground them, I introduced the concepts of maintenance and relapse prevention, and described their importance in establishing long-term change. I emphasized that to maintain the gains he made in treatment, he would have to live something of an "exposure lifestyle." He would still need to deliberately engage with the things that made him uncomfortable, practice his ability to tolerate distress, and prevent himself from falling back into avoidance. I also made sure to point out that despite significant progress, several targets remained for exposure if Daniel anted to extinguish as much of his worry as possible, including eating in restaurants and returning to attending school in person.

Daniel jumped at the idea transitioning to the maintenance phase, expressing that he had made a lot of progress and that he did not think of himself as very anxious anymore. I wondered out loud if the last few weeks of not monitoring fully possibly reflected how he felt about his OCD and therapy at this point. After I expressed I would not take anything personally, he expressed some fatigue with treatment. My role in treatment is guide or coach, so while I 
reiterated my concerns about the things he still felt anxious about (emphasizing his avoidance of in-person school), I also encouraged him to make the decision with his parents that felt best for him. In this case, Daniel and his father felt he was ready to move to a maintenance phase of treatment and reduce the frequency of sessions. In practice, Daniel would stop monitoring his rituals or planning specific exposures, while shifting to that "exposure lifestyle." Daniel's father agreed to keep pushing him to do all of the normal daily life tasks, as well as weekly activities or outings outside of his comfort zone. We agreed to test out this maintenance phase for an initial two-week period to determine if he would be able to manage without slipping back.

After Daniel and his father had left the session, and I took my regular detailed reflection notes about the session content and its process. In this moment, it was important to consider how much energy and time E/RP requires each week, for both the family overall and especially for a 14-year-old. I also had to reflect on whether my own goals for Daniel, such as complete OCD symptom remission and a return to a normal school environment, might not match Daniel goals. For that matter, my goals might not match his parents' goal of returning him to a developmentally appropriate lifestyle, even if that included some mild worry or avoidance. I reconsidered my perceptions of what a good or positive treatment outcome for Daniel could be, including schoolrelated treatment outcomes. With this opportunity to reflect, I was able to see more clearly that the shift to a maintenance phase had been building for the past weeks, and that Daniel had more or less returned to the life he lived prior to the development of the OCD.

After two weeks, Daniel, his parents, and I met for Session 23 (June 13), beginning with a discussion of how the shift to the maintenance phase of treatment had gone for Daniel and the family. They reported that Daniel had pushed himself in the past couple of weeks, despite removing some of the scaffolding that had supported him. Both Daniel and his parents described that he had continued to engage with the daily activities that had been avoided prior to his repeated exposure as part of his treatment, such as going to stores, eating out at restaurants, spending time with friends, flushing the toilet and putting down the seat, doing the laundry, and loading the dishwasher.

They also painted the picture of a more typical teenager, going through typical adolescent activities without being significantly preoccupied by intrusive thoughts, feeling the urge to avoid, or for that matter becoming overwhelmed with his distress and trying to escape. Daniel also reported that he was still only washing his hands before meals, sometimes forgetting to do that entirely, and that he had stopped washing when he felt anxious.

This discussion transitioned easily into a relapse prevention activity that I created prior to this session, designed to reinforce his knowledge about E/RP, OCD, and more specifically, how to recognize and cope with anxiety in the future. Daniel applied his knowledge and personal experiences to a number of hypothetical scenarios in which he may begin to experience additional intrusive thoughts and compulsive urges. Importantly, Daniel highlighted the importance of identifying and talking about his worries with parents, so that they could support him in resisting and exposing himself. In addition, they could help him restart treatment if there were any concerns about a relapse. We had been practicing similar activities in the last few sessions, with targeted Socratic questioning to push him to verbalize his E/RP and OCD 
Pragmatic Case Studies in Psychotherapy, http://pcsp.libraries.rutgers.edu

Volume 15, Module 1, Article 1, pp. 1-74, 03-22-19 [copyright by author]

knowledge, and he seemed to benefit from it greatly, as he accurately and comprehensively described how he should respond to those things if they occurred.

Next, Daniel and I completed another rating of the CY-BOCS measure, which showed clearly what we had seen in the homework and what we had been discussing in session: Daniel's symptoms had fallen significantly. More specifically, Daniel endorsed an obsession severity score of 4, a compulsion severity score of 5 , for a total severity score of 9 (see Table 1 ). Compared to the CY-BOCS total severity rating of 18 from one month earlier, his current symptoms had fallen significantly, down to the low end of the "Mild" classification range. In fact, this was close to the "Subclinical" range. Thus, it was apparent just how much progress he had made, and that his gains in life functional had translated into steadily lower anxiety. In addition, Daniel's second rating of the Severity Measure of GAD also reflected the sharp reduction in his symptoms since the beginning of treatment, with his score falling from a severe range rating of 3.2 to a mild range rating of 1.20 (see Table 2 and Figure 2).

Finally, I returned our focus to treatment planning, and how I could best support Daniel and his parents. Both Daniel and his parents felt that he was ready to wind down treatment, although his parents did express some understandable concern about symptoms returning. I supported their desire to wind down, while suggesting it might be prudent to keep in touch, and schedule at least one session for a month out. While supporting their decision and suggesting we meet at least once more in a month's time, I also reiterated that I saw some areas where Daniel could still progress, particularly at school.

Unaware of Daniel's functioning over the preceding month, I came to Session 24 prepared with a flexible session plan in mind, based in part on the final two sessions of the E/RP protocol, including the "Graduation Ceremony." We began as usual with a check-in, reviewing the past month in terms of his symptoms, behaviors, and functioning. Daniel and his parents reported that his symptoms had remained stable and still very low, such that they noticed only a few occasional moments of Daniel appearing only mildly worried or concerned about something. Furthermore, his parents noted that they had noticed no hand washing or other rituals during the past month (considering hand washing prior to meals to be appropriate), and that he had not fallen back into any of his past avoidance patterns. I encouraged them to maintain this trend over the rest of the summer by getting him into summer activities as much as possible.

Overall, they were very happy to report that the profound symptom reduction had maintained over the course of the month. I took the opportunity to repeat the maintenance and relapse prevention activity completed in the last session, with Daniel responding to various hypothetical scenarios to assess and reinforce his knowledge and plan. In addition, we completed one last CY-BOCS rating, with Daniel endorsing literally no obsessive or compulsive symptoms: Obsession Severity Score $=0$, Compulsion Severity Score $=0$, Total Symptom Severity Score $=0$ (see Table 1). Such a low number surprised me, but Daniel stood by his ratings and his parents agreed, reporting no anxiety over the past week and no completed rituals. Even now, I am unsure of the accuracy of that rating, considering that Daniel seemed to struggle to report the frequency and intensity of his symptoms at times over the course of treatment. However, that aside, the Subclinical rating of his symptoms was consistent with everything that the family and I saw 
Pragmatic Case Studies in Psychotherapy, http://pcsp.libraries.rutgers.edu

Volume 15, Module 1, Article 1, pp. 1-74, 03-22-19 [copyright by author]

overall. Daniel had made incredible progress working to overcome his OCD, and this progress had remained stable to that point.

Finally, Daniel, his parents, and I spent the rest of the session celebrating Daniel's "Graduation" from therapy, including giving him a certificate to highlight his achievement. Important to note: we had not followed the protocol's recommendation to create a narrative or story of Daniel's life and progress with OCD during treatment, due to his disengagement during the preceding "Mapping OCD" activity and that this narrative appeared aimed toward younger children. However, we took time to look back and reflect on the profound changes that had occurred in his life, and how he achieved his goals through hard work and determination across the entire six months. Daniel and his parents highlighted what they learned along the way, and how they felt equipped to handle future "speed bumps" that he might face. Of course, I took as many opportunities as possible to reinforce the important concepts of E/RP, including the model, rationale, and how to apply it to his daily life moving forward. Moreover, consistent with my approach to the treatment, I also provided Daniel with praise and positive encouragement to continue living the life for which he had fought so hard.

\section{The Therapeutic Relationship Across Treatment}

As with all psychotherapy, the relationship between Daniel and me was critical to the process and outcome of treatment. By its nature, E/RP is a challenging therapy that asks all clients to push themselves out of their comfort zone as frequently as possible. In order to achieve that fundamental goal, I worked to build a strong, open, honest, and trusting relationship with Daniel from the first session. We talked about his interest in sports and videogames, and our sharing of those interests enabled us to quickly develop a good rapport. I was also direct and honest from our first encounter, talking with him about what he was going through and how I knew methods to help him move forward. I believe my clear confidence in the treatment implicitly and explicitly communicated to Daniel that "buying in" would lead to a positive outcome. Throughout treatment, I made the effort to emphasize the importance of honesty, while avoiding a shaming or punishing attitude, such that mistakes or challenges would be treated as useful information and part of the process.

Over the course of treatment, Daniel's motivation and interest in treatment waxed and waned, and it gradually decreased as we got closer to terminating. This appeared to be a combination of treatment fatigue as well as lowered symptoms leading to a lowered sense of urgency, rather than a comment on our relationship. In fact, at our final session, it seemed clear that Daniel and I had developed a very strong relationship over the course of treatment. Because of the trust that had developed, I was able to utilize that relationship to help push Daniel further out of his comfort zone than he might have been able to do alone. Often, I utilized our strong relationship to more openly and honestly communicate difficult messages, such as that he would not overcome his OCD if he couldn't complete a specific exposure.

At other moments, this involved my strategic use of reward time after a difficult exposure or when he was feeling less than motivated to complete an exposure. As I got to know him better, I came to see when it would benefit Daniel to do something fun outside of the usual 
session activities, if for no other reason but to improve his mood and inject some levity into our sessions. Although reward time was not described in the protocol, we would spend 5 to 10 minutes talking about sports and video games or having him show me things. This adaptation to the protocol was a powerful and effective tool at helping to keep Daniel engaged and motivated in treatment. This, then, is a key aspect of treatment that lies outside of the theoretical treatment model, namely, a strong therapeutic alliance and relationship are a critical component to successful treatment, and without it, the chances of the patient's symptoms remitting entirely are low.

\section{Remote Video Sessions}

The setting of Daniel's therapy was another interesting component of his treatment, considering that roughly $20 \%$ of his sessions were remote sessions utilizing video conferencing technology (see Table 10). These remote video sessions had a number of strengths and weaknesses in comparison to more traditional in-person sessions at the office. Compared to having Daniel in the office with me, video sessions naturally provided less information, whether it was non-verbal behavior or simply action happening outside of the camera's view. In terms of strengths, this technology helped facilitate an increase in session frequency greater than what the family would have been able to manage without it, specifically due to the long drive to the clinic's offices. In addition to enabling a greater number of sessions overall, Daniel and I were able to complete real time assessment and exposures in his home, which is one of the best environments to do so because of how much time he spends there. It also allowed for a greater level of monitoring and accountability, as I could directly see whether he was able to complete the exposures I was assigning as homework.

\section{THERAPY MONITORING AND USE OF FEEDBACK INFORMATION}

I monitored Daniel's progress with treatment on a weekly, and often twice-weekly basis, using the additional structure and scaffolding described at the beginning of the Course of Therapy section. This included in-depth plans prior to sessions, detailed in-session notes, post-session critical reflection notes about the session and treatment process, audio recordings, and supervision with Dr.

Franklin. During supervision, I would provide a recap of the previous session, and come prepared with an updated conceptualization as necessary, as well as a tentative plan for the upcoming session. Dr. Franklin's knowledge of and experience with OCD, E/RP, and Daniel's individual presentation helped us to determine appropriate and effective adaptations of the protocol, such as how and when to include his parents in session, or how to motivate Daniel to push through his avoidance.

One major psychometric measure, the CY-BOCS, was also used to track Daniel's symptoms over the course of nine months, from two months prior to treatment until the end of treatment (see Table 1). We also used an additional measure, his Subjective Units of Distress (SUDS) or, as well called it, his "temperature" rating, throughout our sessions and exposures to keep track of his levels of anxiety on a moment-to-moment basis. Of course, as discussed at 
length above, Daniel (and sometimes his mother) kept a detailed record of his efforts at exposure and ritual prevention over much of treatment. While the CY-BOCS allowed us to measure his progress in treatment over time, Daniel's rating of his "temp" was a crucial tool for facilitating exposures, especially being able to determine what would be the next most challenging task he was willing to engage with. In addition, the Severity Measure for GAD--Child age 11-17, DSM-5 (see Table 2) was used as an initial tool to confirm severity of non-OCD symptoms and ultimately as an outcome measure, to show Daniel just how much progress he had made in treatment.

\section{CONCLUDING EVALUATION OF THE THERAPY'S PROCESS AND OUTCOME}

\section{The Successful Process of Daniel's Therapy}

As evidenced in the treatment narrative, Daniel entered treatment struggling with severe anxiety and distress to such an extent that he withdrew into a very limited lifestyle. His intrusive thoughts about contamination and becoming ill seemed to coincide with an avoidance-based coping style, which unfortunately led to a vicious cycle of negative reinforcement of his worries based on the repeated completion of rituals to manage his distress. From a functional perspective, Daniel's OCD and GAD symptoms led him to pull back from many developmentally appropriate, daily activities and tasks, including an eventual withdrawal from a more traditional "brick and mortar" school environment. Moreover, Daniel's worries about germs prevented him from spending time with friends or participating in appropriate social activities, including with his family.

The Exposure and Response Prevention (E/RP) treatment involved teaching Daniel to understand OCD and how his behaviors can strengthen or weaken its symptoms, depending on his choices. Daniel fought to overcome his significant anticipatory anxiety, before directly and deliberately engaging with his greatest fears, in the form of stimuli or situations that he worried about or avoided. In fact, rehearsal of the E/RP model and rationale was an important aspect of the therapy, as it reinforced the critical importance of Daniel not allowing himself to escape or avoid that which scared him. Daniel then had to directly confront his fears (exposure), which provided him with repeated opportunities to debunk his hypotheses regarding his obsessions and the associated feared consequences, and in turn demonstrating to himself that he is capable of tolerating significant levels of distress. In addition, it is important to note that by the end of treatment, as Daniel's OCD symptoms had abated, his GAD worries had also fallen as well, to the point that Daniel no longer met criteria for either diagnosis (see Table 5).

\section{Qualitative Results}

As described in the description of Session 24, our final appointment after a month of no sessions, Daniel, his parents, and I explored in-depth the ways that Daniel's life had functionally changed over the course of treatment. We all could agree that his hard work and commitment to the in-session tasks and homework helped him achieve significant symptom remission, despite the challenges of E/RP. Furthermore, Daniel regained the ability, or willingness, to engage with the 
aspects of life that he had been avoiding for upwards of two years, re-learning the extent of his true capabilities. By the end of treatment, Daniel described spending more and more time with neighborhood friends, as well as going to restaurants, stores, the beach, movies, and even the zoo, with his family. Importantly, Daniel not only "regained" those activities and places, but also described engaging in these things without significant distress or anxiety. Thus, from a qualitative perspective, Daniel had achieved all of the functional goals identified by the family at the onset of therapy.

It is critical to note that Daniel's progress in treatment did not follow a linear path, with multiple highs, lows, and a number of plateaus over the six months of treatment. At times, Daniel understandably struggled to change long-established patterns of behavior and maintain his motivation, particularly in the context of a disorder and treatment that requires substantial treatment time and effort for adolescents and their families. However, in the end, those efforts paid off, and Daniel and his family achieved their primary goal: returning Daniel to the lifestyle he enjoyed prior to the development of his anxiety - full of friends, family, and fun - rather than a life containing extensive worry and preoccupation.

\section{Quantitative Results}

Daniel presented to COTTAGe approximately two months prior to the start of treatment to complete a comprehensive diagnostic evaluation with me, prior to being taken off the waitlist and assigned to my caseload. As shown in Table 1, Daniel's CY-BOCS rating of his OCD symptoms at this initial intake evaluation indicated symptoms that fell within the high end of the "Severe" range (Total Symptom Severity Score $=31$ ). At Session 2 of treatment, approximately two and a half months later, as well as a month into treatment at Session 7, Daniel's OCD symptoms remained in the "Severe" range (Total Symptom Severity Scores $=25$ and 26, respectively; see Table 1 and Figure 1). At our next rating during Session 13, his symptoms did reflect the significant progress he had been making in treatment as his Total Severity Score fell to the low end of the "Moderate" range (Total Symptom Severity Score = 17). Consistent with the plateau's seen earlier in treatment, roughly one month later, at Session 19, Daniel's symptoms remained in the "Moderate" Range (Total Symptom Severity Score =18).

Although we continued to see significant positive strides in treatment, Daniel's symptoms did not reflect that progress until our next rating a month later at Session 23. Specifically, Daniel's CY-BOCS Total Severity Score had fallen drastically, into the "Mild" range of symptoms (Total Symptom Severity Score $=9$ ). Based on Daniel's progress with exposures and considering this precipitous drop in symptoms, the treatment shifted to the Maintenance and Relapse Prevention phase of treatment. After approximately one month, Daniel's final rating of his OCD symptoms at Session 24 reflected his belief that he no longer spent time preoccupied with intrusive thoughts or engaged in any compulsive behaviors (Total Symptom Severity Score $=0$; see Table 1 and Figure 1). In terms of his GAD symptoms, Daniel experienced a significant drop in symptoms from a severity of 3.2 at Session 2, to a severity of 1.2 at Session 23, representing a shift from the Severe range to the Mild range (see Figure 2). The reduction in severity of his GAD parallels the significant drop in his OCD, although this measure was not administered with the same frequency as the CY-BOCS. 
Pragmatic Case Studies in Psychotherapy, http://pcsp.libraries.rutgers.edu

Volume 15, Module 1, Article 1, pp. 1-74, 03-22-19 [copyright by author]

\section{Outcome Relative to Initial Goals}

Daniel and his parents identified the following goals at the outset of treatment:

1. Reduce frequency of compulsive behaviors;

2. Learn to tolerate or cope with anxiety and distress; and

3. Improve functioning, such as increasing engagement in developmentally appropriate tasks inside the home and social activities outside of the home.

Based on the quantitative results described above, Daniel successfully achieved both Goal \#1 and Goal \#2, leaving treatment with significantly lowered levels of obsessions and compulsions. However, most important to his parents, Daniel achieved Goal \#3 with flying colors, as he returned to a level of functioning very similar to his baseline level of functioning prior to the development of his anxiety. In practical terms, Daniel recovered his willingness to do almost all of the things he had been avoiding, such as going into stores, eating in restaurants, playing with friends, and going to crowded places (e.g. the zoo or a movie). These changes not only represented a significant regaining of whole aspects of life for Daniel, but they also meant that the entire family could return to a more typical lifestyle, one that did not involve persistent avoidance of people, places, activities, and situations.

\section{Discussion}

Overall, Exposure and Response Prevention represented the most appropriate and evidence-based treatment for Daniel's OCD and GAD. Over the course of the six months of therapy, Daniel came to internalize the core message of E/RP: in order to reduce his distress and learn to tolerate his worries he would have to directly engage with and expose himself to those things, while simultaneously preventing himself from coping via rituals or compulsions. The next step in this line of thinking suggests that to prevent an OCD relapse, with the support of his family, Daniel should live a lifestyle that prioritizes deliberate confrontation of fears and rejects avoidance.

As described above, the course of treatment and Daniel's progress did not follow a linear path. His effort and engagement in treatment rose and fell depending upon the specific exposure at hand, circumstances of his life during that week, and even depending upon the point in the overall course of treatment. For example, there were a few plateaus in symptom reduction during the course of treatment, including between the CY-BOCS ratings at Sessions 13 and 19, i.e., sessions between 4/14/16 and 5/16/16 (Figure 1). Interestingly, when looking at Figures 3 and 4, one can also see that during roughly the same time Daniel's daily rituals increased in frequency while his ritual prevention decreased. That pattern seems to strengthen the link between the severity of Daniel's OCD and the frequency of his use of rituals to cope.

To work through the obstacles arising throughout treatment and to achieve a positive outcome, flexibility was critical, while maintaining fidelity to the E/RP principles. Because OCD symptoms are complex, it can be hard for children and adolescents to verbalize their worries, let 
alone describe their complicated, distressing thoughts or behaviors. Daniel struggled with this, so I had to adjust my approach to incorporate assessment as a consistent part of each session. I attempted to bring into each session an attitude of being curious and not avoiding asking the difficult questions. Second, pediatric OCD treatment requires the clinician to be able to apply flexibly the principles of E/RP to a variety of presentations because each specific patient can vary so significantly in his or her of thoughts of worry and resulting behaviors.

Another significant obstacle in treatment was Daniel's variable motivation to complete the important tasks of E/RP, especially given his deeply ingrained avoidance habits. As a result, the family and I had to make a number of changes to provide Daniel with greater structure and support to bolster his confidence, and in turn, bolster his willingness to engage with things that his mind and body were telling him to continue to avoid, especially as the difficulty-level of exposures increased. This included adding successively greater parental involvement in and out of the sessions, increasing the specificity of the rewards he earned, and linking those rewards more closely to his completion of exposures for homework. We linked rewards to not only exposure completion, but also consistent monitoring of his ritual prevention tasks. In addition, as treatment continued, I also reconsidered my conceptualization of what would constitute a good treatment outcome for Daniel, and realized that treatment did not need to be linear for it to lead to a profoundly positive outcome for him and his family.

Furthermore, we had to be flexible and employ a series of strategic adaptations to the manual to improve the applicability to Daniel, his symptoms, and his life. Overall, we did not follow the protocol's specific session plans after the few initial sessions, based on the fact that certain components required less time to achieve progress, for example the exposures, compared to other components, that required more time in and out of session to fully complete (see Table 10). For our adjustments to Daniel's rewards, while the manual describes the importance of rewards for one session, it does not provide much additional detail into how to establish such a system, or how to use it to encourage progress in treatment. In addition, the manual calls for a specific number of parent-only sessions, to provide them psychoeducation, complete assessment, and coaching on how how to assist Daniel. However, rather than discrete parent-only sessions, we structured the sessions to involve the parents as frequently as possible, often including them in either the beginnings or ends of sessions, if they were not present for the entirety.

Other deviations from the manual included the fact that I did not incorporate a number of specific interventions designed for a younger pediatric population, rather than a 14-year-old, albeit one who was still in middle school. In order to adapt to his age and developmental level, we did not utilize the "Mapping the OCD" metaphor or technique after the initial introduction. This may have been due to my perceptions of Daniel's initial level of engagement with the "Mapping" activity; in any event, it did not seem to match Daniel or his needs. Similarly, the manual recommends utilizing a narrative or story metaphor in part to strengthen the child's learning, and to integrate that learning into a connected "story" of their lives. Although very helpful for younger children, this did not seem to be appropriate for Daniel's age, developmental level, and his interests, as it did not seem advanced enough as presented in the manual. Similar to the topic of use of rewards, this manual provided only broad information and left out specific details about how to implement or structure the "Mapping" activity. Understandably this made it difficult to 
utilize this activity, especially while trying to adapt the manual to a real patient.

Nevertheless, Daniel's treatment largely followed the manual, particularly when considering the general principles of exposure and response prevention and the approximate structure of each session. As described above, the majority of our sessions followed this format: 1) check-in since last session and homework review; 2) in-vivo exposures or exposure problemsolving-and-planning; and 3) planning homework (see Table 6). Maintaining this session structure allowed me to provide a consistent therapeutic environment, in which Daniel knew what to expect in the next clinical encounter. Most of the remote video sessions also maintained this format, and while I was not physically present with him for exposures, Daniel completed them in-vivo, in real time, as I assisted and monitored through the video feed.

In conclusion, Daniel's case study is designed to provide the reader with an understanding of how to flexibly apply the principles of Exposure and Response Prevention to address a complex presentation of OCD and GAD symptoms. Daniel's case study also enables the reader to consider some of the strengths and weaknesses of an evidence-based treatment protocol, as well as how a therapist can adjust the in-session and out-of-session interventions to mitigate those weaknesses. The protocol's primary weakness lies in the lack of sufficient specificity in its description of various interventions. In practical terms, it means novice or intermediate clinicians will struggle to implement the techniques independently, without a lot of previous knowledge or experienced supervisors. The manual could also be improved in terms of how clearly it links theory to the exposure of certain obsessions and compulsions. For example, the protocol only talks about general types of exposures. It would be clinically useful for the manual to provide the reader with sample exposures for specific obsession subtypes, such as symmetry, "just right," and contamination.

As it relates to those weaknesses in the manual and the concept of "flexibility within fidelity," all of the adjustments to the protocol were made to address Daniel's specific symptoms and needs. As early as Phase I (Assessment) and as part of the guiding conception of this case study, I expected that we would need to make significant adaptations to E/RP compared with how it is laid out in the manual, in order to fully extinguish all of Daniel's worries, given his comorbid severe non-compulsive worries (i.e., his GAD) at the beginning of treatment (see Table 2). In fact, during the Assessment Phase of treatment, Daniel and I brainstormed and included all of his noncompulsive worries on the hierarchy, intending to expose him to all of those relevant triggers after his OCD symptoms lessened.

However, to our surprise, Daniel's non-compulsive worries remitted fully without significant additional exposures to target those specific worries. Similar to a Venn diagram, it appeared that Daniel's GAD and OCD symptoms had enough overlap that the underlying worries were exposed at the same time, even when he was not directly exposed to the triggers of his noncompulsive worries.

Because Daniel was not administered the GAD severity measure at the same frequency as the CY-BOCS, the current analysis lacks the same level of detail regarding symptom fluctuation over time. However, looking back over the course of treatment, Daniel gained crucial inhibitory 
learning that applied to both his contamination obsessions and to his non-compulsive worries about health and safety. However, it was not clear at the beginning, or even in the middle of treatment, that we would not have to specifically expose him to his non-compulsive worries. It only became clear near the end of treatment that his GAD had remitted in step with his OCD. This result suggests a few final things about the protocol and about E/RP more broadly. First, it seems the adaptations made to the protocol primarily addressed Daniel's needs and his specific symptoms, rather than addressing comorbidity per se. That being said, based on the case of Daniel, a clinician should expect that changes to the protocol will be needed, along with a flexible and persistent attitude, in order to remain in line with the core principles of E/RP and to address the issues with which that particular patient presents, above and beyond their primary diagnosis. Second, Daniel's profound progress and functional improvement provides a great example of the strength and robustness of exposure and response prevention treatments for OCD and anxiety, while also highlighting some of the remaining weaknesses in the treatment materials used by clinicians. As this case reveals, clinicians focusing on the fundamental principles of E/RP, while being flexible to meet the patient's specific needs, can successfully utilize this treatment even for patients like Daniel, with severe and complex presentations.

\section{REFERENCES}

Abramowitz, J. S., Franklin, M. E., \& Foa, E. B. (2002). Empirical status of cognitive-behavioral therapy for obsessive-compulsive disorder: A meta-analytic review. Romanian Journal of Cognitive \& Behavioral Psychotherapies.

Abramowitz, J. S., Whiteside, S. P., \& Deacon, B. J. (2006). The effectiveness of treatment for pediatric obsessive-compulsive disorder: A meta-analysis. Behavior Therapy, 36 (1), 5563.

Albano, A.M., \& Silverman, W.K. (1996). Anxiety Disorders Interview Schedule for DSM-IV: Child Version. Oxford, UK: Oxford University Press.

American Psychiatric Association. (2013). Diagnostic and statistical manual of mental disorders (DSM-5®). American Psychiatric Pub.

American Psychiatric Association (2018). Severity Measure for Generalized Anxiety DisorderChild Age 11-17, DSM-5. Washington, D.C.: American Psychiatric Association. https://www.psychiatry.org/psychiatrists/practice/dsm/educational-resources/assessment$\underline{\text { measures }}$

Andrews, G., Stewart, G., Morris-Yates, A., Holt, P., \& Henderson, S. (1990). Evidence for a general neurotic syndrome. The British Journal of Psychiatry, 157(1), 6-12.

Baldwin, D. S., Ajel, K. I., \& Garner, M. (2009). Pharmacological treatment of generalized anxiety disorder. In Behavioral Neurobiology of Anxiety and Its Treatment (pp. 453-467). Springer, Berlin, Heidelberg.

Barlow, D. H. (2002). Anxiety and its disorders. Guildford Press.

Craske, M. G., Kircanski, K., Zelikowsky, M., Mystkowski, J., Chowdhury, N., \& Baker, A. (2008). Optimizing inhibitory learning during exposure therapy. Behaviour research and therapy, 46(1), 5-27.

Cuijpers, P., Sijbrandij, M., Koole, S., Huibers, M., Berking, M., \& Andersson, G. (2014). Psychological treatment of generalized anxiety disorder: a meta-analysis. Clinical psychology review, 34(2), 130-140. 
Douglass, H. M., Moffitt, T. E., Dar, R., McGee, R. O. B., \& Silva, P. (1995). Obsessivecompulsive disorder in a birth cohort of 18 -year-olds: prevalence and predictors. Journal of the American Academy of Child \& Adolescent Psychiatry, 34(11), 1424-1431.

Edwards, S. L., Rapee, R. M., \& Kennedy, S. (2010). Prediction of anxiety symptoms in preschool-aged children: examination of maternal and paternal perspectives. Journal of Child Psychology and Psychiatry, 51(3), 313-321.

Fals-Stewart, W., Marks, A. P., \& Schafer, J. (1993). A comparison of behavioral group therapy and individual behavior therapy in treating obsessive-compulsive disorder. Journal of Nervous and Mental Disease. 181(3), 189-193

Fishman, D. (1999). The case for pragmatic psychology. New York: New York University Press.

Fishman, D. B. (2005). Editor's introduction to PCSP-From single case to database. Pragmatic Case Studies in Psychotherapy, 1(1), Article 2, 1-50. Available: http://pcsp.libraries.rutgers.edu. http://dx.doi.org/10.14713/pcsp.v1i1.855

Fishman, D. B. (2013). The pragmatic case study method for creating rigorous and systematic, practitioner-friendly research. Pragmatic Case Studies in Psychotherapy, 9(4), Article 2, 403-425, Available: http://pcsp.libraries.rutgers.edu. http://dx.doi.org/10.14713/pcsp.v9i4.1833

Flament, M. F., \& Cohen, D. (2000). Child and adolescent obsessive-compulsive disorder: a review. Obsessive-compulsive disorder, 147-183.

Foa, E. B., Grayson, J. B., Steketee, G. S., Doppelt, H. G., Turner, R. M., \& Latimer, P. R. (1983). Success and failure in the behavioral treatment of obsessive-compulsives. Journal of consulting and clinical psychology, 51(2), 287.

Foa, E. B., \& Kozak, M. J. (1985). Treatment of anxiety disorders: Implications for psychopathology.

Foa, E. B., \& Kozak, M. J. (1986). Emotional processing of fear: exposure to corrective information. Psychological bulletin, 99(1), 20.

Franklin, M. E., Abramowitz, J. S., Kozak, M. J., \& Levitt, J. T. Foa. EB (2000). Effectiveness of exposure and ritual prevention for obsessivecompulsive disorder: Randomized compared with nonrandomized samples. Journal of Consulting and Clinical Psychology, 68(4), 594-602.

Franklin, M., Foa, E., \& March, J. S. (2003). The pediatric obsessive-compulsive disorder treatment study: rationale, design, and methods. Journal of Child and Adolescent Psychopharmacology, 13(2, Supplement 1), 39-51.

Franklin, M., Kratz, H., Freeman, J., Ivarsson, T., Heyman, I., Sookman, D., McKay, D., Storch, E., March, J. (2015). Cognitive-behavioral therapy for pediatric obsessive-compulsive disorder: empirical review and clinical recommendations. Psychiatry Research, 227, 7892.

Franklin, M. E., Sapyta, J., Freeman, J. B., Khanna, M., Compton, S., Almirall, D., ... \& Foa, E. B. (2011). Cognitive behavior therapy augmentation of pharmacotherapy in pediatric obsessive-compulsive disorder: the Pediatric OCD Treatment Study II (POTS II) randomized controlled trial. Journal of the American Medical Association, 306(11), 1224-1232. 
Pragmatic Case Studies in Psychotherapy, http://pcsp.libraries.rutgers.edu

Volume 15, Module 1, Article 1, pp. 1-74, 03-22-19 [copyright by author]

Goodman, W.K., Price, L.H., Rasmussen, S.A., Mazure, C., Fleischmann, R.L., Hill, C.L., Heninger, G.R., \& Charney, D.S. (1989a). The Yale-Brown Obsessive Compulsive Scale: I. Development, use, and reliability. Archives of General Psychiatry 46, 1006 1011.

Goodman, W.K., Price, L.H., Rasmussen, S.A., Mazure, C., Delgado, P., Heninger, G.R., \& Charney, D.S. (1989b). The Yale- Brown Obsessive Compulsive Scale: II. Validity. Archives of General Psychiatry 46, 1012-1016.

Jacoby, R. J., \& Abramowitz, J. S. (2016). Inhibitory learning approaches to exposure therapy: A critical review and translation to obsessive-compulsive disorder. Clinical psychology review, 49, 28-40.

Kessler, R. C., Petukhova, M., Sampson, N. A., Zaslavsky, A. M., \& Wittchen, H. U. (2012). Twelve-month and lifetime prevalence and lifetime morbid risk of anxiety and mood disorders in the United States. International journal of methods in psychiatric research, 21(3), 169-184.

Lang, P. J. (1971). The application of psychophysiological methods to the study of psychotherapy and behavior modification. Handbook of psychotherapy and behavior change, 75-125.

Lang, P. J., Craske, M. G., \& Bjork, R. A. (1999). Implications of a new theory of disuse for the treatment of emotional disorders. Clinical Psychology: Science and Practice, 6(1), 80-94.

Lindsay, M., Crino, R., \& Andrews, G. (1997). Controlled trial of exposure and response prevention in obsessive-compulsive disorder. The British Journal of Psychiatry, 171(2), 135-139.

Maj, M., Sartorius, N., Okasha, A., \& Zohar, J. (Eds.). (2003). Obsessive-compulsive disorder. John Wiley \& Sons.

March, J. S., \& Mulle, K. (1994). How I ran OCD off my land: A guide to cognitive-behavioral psychotherapy for children and adolescents with obsessive-compulsive disorder. Durham, NC: Duke University Medical Center.

March, J. S., \& Mulle, K. (1998). OCD in children and adolescents: A cognitive-behavioral treatment manual. Guilford Press.

March, J., Mulle, K., Foa, E., \& Kozak, M. (2002) Treatment of Pediatric Obsessive-Compulsive Disorder Cognitive-Behavioral Therapy Treatment Manual. Unpublished manuscript.

Marks, I. M., Stern, R. S., Mawson, D., Cobb, J., \& McDonald, R. (1980). Clomipramine and exposure for obsessive-compulsive rituals: i. The British Journal of Psychiatry, 136(1), 125.

Mash, E. J., \& Barkley, R. A. (Eds.). (2014). Child psychopathology. Guilford Publications.

Masi, G., Millepiedi, S., Mucci, M., Poli, P., Bertini, N., \& Milantoni, L. (2004). Generalized anxiety disorder in referred children and adolescents. Journal of the American Academy of Child \& Adolescent Psychiatry, 43(6), 752-760.

Mataix-Cols, D., Nakatani, E., Micali, N., \& Heyman, I. (2008). Structure of obsessivecompulsive symptoms in pediatric OCD. Journal of the American Academy of Child \& Adolescent Psychiatry, 47(7), 773-778.

Meyer, V. (1966). Modification of expectations in cases with obsessional rituals. Behaviour research and therapy, 4(4), 273-280.

Mowrer, O. (1960). Learning theory and behavior. New York: Wiley. 
Pragmatic Case Studies in Psychotherapy, http://pcsp.libraries.rutgers.edu

Volume 15, Module 1, Article 1, pp. 1-74, 03-22-19 [copyright by author]

Newman, M. G., \& Llera, S. J. (2011). A novel theory of experiential avoidance in generalized anxiety disorder: A review and synthesis of research supporting a contrast avoidance model of worry. Clinical psychology review, 31(3), 371-382.

Pediatric, O. C. D. (2004). Cognitive-behavior therapy, sertraline, and their combination for children and adolescents with obsessive-compulsive disorder: the Pediatric OCD Treatment Study (POTS) randomized controlled trial. Jama, 292(16), 1969.

Pine, D. S., Helfinstein, S. M., Bar-Haim, Y., Nelson, E., \& Fox, N. A. (2009). Challenges in developing novel treatments for childhood disorders: lessons from research on anxiety. Neuropsychopharmacology, 34(1), 213.

Power, K. G., Simpson, R. J., Swanson, V., Wallace, L. A., Feistner, A. T. C., \& Sharp, D. (1990). A controlled comparison of cognitive-behaviour therapy, diazepam, and placebo, alone and in combination, for the treatment of generalised anxiety disorder. Journal of Anxiety Disorders, 4(4), 267-292.

Purdon, C., \& Clark, D. A. (2002). The need to control thoughts. In Cognitive approaches to obsessions and compulsions (pp. 29-43).

Rachman, S. (1998). A cognitive theory of obsessions. In Behavior and Cognitive Therapy Today (pp. 209-222).

Rothbaum, B. O., \& Shahar, F. (2000). Behavioral treatment of obsessive-compulsive disorder in a naturalistic setting. Cognitive and Behavioral Practice, 7(3), 262-270.

Salkovskis, P. M. (1985). Obsessional-compulsive problems: A cognitive-behavioural analysis. Behaviour research and therapy, 23(5), 571-583.

Scahill, L., Riddle, M., McSwiggin-Hardin, M., Ort, S., King, R., Goodman, W., Cicchetti D., Leckman, J.F. (1997). Children's Yale-Brown obsessive compulsive scale: reliability and validity. Journal of American Academy of Child and Adolescent Psychiatry, 36, 844-852.

Selles, R. R., Franklin, M., Sapyta, J., Compton, S. N., Tommet, D., Jones, R. N., ... \& Freeman, J. (2017). Children's and parents' ability to tolerate child distress: impact on cognitive behavioral therapy for pediatric obsessive compulsive disorder. Child Psychiatry \& Human Development, 1-9.

Skarphedinsson, G., De Nadai, A. S., Storch, E. A., Lewin, A. B., \& Ivarsson, T. (2017). Defining cognitive-behavior therapy response and remission in pediatric OCD: a signal detection analysis of the Children's Yale-Brown Obsessive Compulsive Scale. European child \& adolescent psychiatry, 26(1), 47-55.

Steketee, G., Chambless, D.L., \& Tran, G.Q. (2001). Effects of axis I and II comorbidity on behavior therapy outcome for obsessive-compulsive disorder and agoraphobia. Comprehensive Psychiatry, 42, 76-86.

Storch, E. A., Geffken, G. R., Merlo, L. J., Jacob, M. L., Murphy, T. K., Goodman, W. K., \& Grabill, K. (2007). Family accommodation in pediatric obsessive-compulsive disorder. Journal of Clinical Child and Adolescent Psychology, 36(2), 207-216.

Storch, E. A., Geffken, G. R., Merlo, L. J., Mann, G., Duke, D., Munson, M., \& Goodman, W. K. (2007). Family-based cognitive-behavioral therapy for pediatric obsessive-compulsive disorder: Comparison of intensive and weekly approaches. Journal of the American Academy of Child \& Adolescent Psychiatry, 46(4), 469-478. 
Storch, E. A., Merlo, L. J., Larson, M. J., Bloss, C. S., Geffken, G. R., Jacob, M. L., \& Goodman, W. K. (2008). Symptom dimensions and cognitive-behavioural therapy outcome for pediatric obsessive-compulsive disorder. Acta Psychiatrica Scandinavica, $117(1), 67-75$.

Vasey, M. W., \& Dadds, M. R. (Eds.). (2001). The developmental psychopathology of anxiety. Oxford University Press.

Warren, R., \& Thomas, J. C. (2001). Cognitive-behavior therapy of obsessive-compulsive disorder in private practice: An effectiveness study. Journal of Anxiety Disorders, 15(4), 277-285.

Weems, C. F., Silverman, W. K., \& La Greca, A. M. (2000). What do youth referred for anxiety problems worry about? Worry and its relation to anxiety and anxiety disorders in children and adolescents. Journal of Abnormal Child Psychology, 28(1), 63-72.

Williams, M. T., Farris, S. G., Turkheimer, E. N., Franklin, M. E., Simpson, H. B., Liebowitz, M., \& Foa, E. B. (2014). The impact of symptom dimensions on outcome for exposure and ritual prevention therapy in obsessive-compulsive disorder. Journal of anxiety disorders, 28(6), 553-558.

Zohar, A. H. (1999). The epidemiology of obsessive-compulsive disorder in children and adolescents. Child and adolescent psychiatric clinics of North America. 
Pragmatic Case Studies in Psychotherapy, http://pcsp.libraries.rutgers.edu

Volume 15, Module 1, Article 1, pp. 1-74, 03-22-19 [copyright by author]

Table 1. Children's Yale-Brown Obsessive Compulsive Scale (CY-BOCS)

\begin{tabular}{|l|l|l|l|l|l|l|l|}
\hline & $\begin{array}{l}\text { Intake } \\
(11 / 15 / 16)\end{array}$ & $\begin{array}{l}\text { Session } \\
\mathbf{1} \\
(1 / 25 / 16)\end{array}$ & $\begin{array}{l}\text { Session } \\
\mathbf{7} \\
(3 / 7 / 16)\end{array}$ & $\begin{array}{l}\text { Session } \\
\mathbf{1 3} \\
(4 / 14 / 16)\end{array}$ & $\begin{array}{l}\text { Session } \\
\mathbf{1 9} \\
(5 / 16 / 16)\end{array}$ & $\begin{array}{l}\text { Session } \\
\mathbf{2 3} \\
(6 / 13 / 16)\end{array}$ & $\begin{array}{l}\text { Session 24 } \\
(7 / 18 / 16)\end{array}$ \\
\hline $\begin{array}{l}\text { Obsession } \\
\text { Symptom } \\
\text { Severity } \\
\text { Score }\end{array}$ & 16 & 13 & 13 & 10 & 9 & 4 & 0 \\
\hline $\begin{array}{l}\text { Compulsive } \\
\text { Symptom } \\
\text { Severity } \\
\text { Score }\end{array}$ & 15 & 12 & 13 & 7 & 9 & 5 & 1 \\
\hline $\begin{array}{l}\text { Total } \\
\text { Symptom } \\
\text { Severity } \\
\text { Score }\end{array}$ & 31 & 25 & 26 & 17 & 18 & 9 & 1 \\
\hline $\begin{array}{l}\text { Total } \\
\text { Severity } \\
\text { Ranges }\end{array}$ & Severe & Severe & Severe & Moderate & Moderate & Mild & Subclinical \\
\hline
\end{tabular}

*Total Severity score ranges groups for patients are as follows: 0-7 (Subclinical), 8-15 (Mild), 16-23 (Moderate), 24-31 (Severe), 32-40 (Extreme)

Table 2. Severity Measure for Generalized Anxiety Disorder - Child Age 11-17 (from DSM-5)

\begin{tabular}{|l|l|l|}
\hline & $\begin{array}{l}\text { Session 2 } \\
(2 / 1 / 16)\end{array}$ & $\begin{array}{l}\text { Session 23 } \\
(6 / 13 / 16)\end{array}$ \\
\hline $\begin{array}{l}\text { Average Total } \\
\text { Score }\end{array}$ & 3.2 & 1.2 \\
\hline $\begin{array}{l}\text { Severity } \\
\text { Ranges }\end{array}$ & Severe & Mild \\
\hline
\end{tabular}

*Average Total Score ranges from 0-4: none (0), mild (1), moderate (2), severe (3), extreme (4) 
Pragmatic Case Studies in Psychotherapy, http://pcsp.libraries.rutgers.edu

Volume 15, Module 1, Article 1, pp. 1-74, 03-22-19 [copyright by author]

Table 3. Results at the Beginning of Treatment from Anxiety Disorders Interview Schedule for DSM-IV-TR (ADIS-IV-TR): Obsessive-Compulsive Disorder (OCD)

\begin{tabular}{|ll}
\hline Criterion & Description \\
\hline A & $\begin{array}{l}\text { Presence of obsessions, compulsions, or both: } \\
\text { Obsessions as defined by (1), (2), (3), and (4): }\end{array}$
\end{tabular}

(1) recurrent and persistent thoughts, impulses, or images that are experienced, at some time during the disturbance, as intrusive and inappropriate and that cause marked anxiety or distress

(2) the thoughts, impulses, or images are not simply excessive worries about real-life problems

(3) the person attempts to ignore or suppress such thoughts, impulses, or images, or to neutralize them with some other thought or action

(4) the person recognizes that the obsessional thoughts, impulses,

Criteria Met or images are a product of his or her own mind (not imposed from without as in thought insertion)

Compulsions as defined by (1) and (2):

(1) repetitive behaviors (e.g., hand washing, ordering, checking) or mental acts (e.g., praying, counting, repeating words silently) that the person feels driven to perform in response to an obsession, or according to rules that must be applied rigidly

(2) the behaviors or mental acts are aimed at preventing or reducing distress or preventing some dreaded event or situation; however, these behaviors or mental acts either are not connected in a realistic way with what they are designed to neutralize or prevent or are clearly excessive

B At some point during the course of the disorder, the person has recognized that the obsessions or compulsions are excessive or unreasonable. Note: This does not apply to children.

C The obsessions or compulsions cause marked distress, are time consuming (take more than 1 hour a day), or significantly interfere with the person's normal routine, occupational (or academic) functioning, or usual social activities or relationships.

D If another Axis I disorder is present, the content of the obsessions or compulsions is not restricted to it

E The disturbance is not due to the direct physiological effects of a substance or a general medical condition.

FULL CRITERIA MET? 


\section{A.M.B. Tice}

Pragmatic Case Studies in Psychotherapy, http://pcsp.libraries.rutgers.edu

Volume 15, Module 1, Article 1, pp. 1-74, 03-22-19 [copyright by author]

Table 4. Results at the beginning of Treatment from Anxiety Disorders Interview Schedule for DSM-IV-TR (ADIS-IV-TR): Generalized Anxiety Disorder (GAD)

\begin{tabular}{|llc|}
\hline Criterion & \multicolumn{1}{c|}{ Description } & Criteria Met \\
\hline A & $\begin{array}{l}\text { Excessive anxiety and worry (apprehensive expectation), } \\
\text { occurring more days than not for at least } 6 \text { months, about a } \\
\text { number of events or activities (such as work or school } \\
\text { performance). }\end{array}$ & $\checkmark$ \\
\hline B & The person finds it difficult to control the worry. \\
\hline C & $\begin{array}{l}\text { C. The anxiety and worry are associated with three (or more) of } \\
\text { the following six symptoms (with at least some symptoms present } \\
\text { for more days than not for the past 6 months). Note: Only one } \\
\text { item is required in children. } \\
\text { (1) restlessness or feeling keyed up or on edge } \\
\text { (2) being easily fatigued } \\
\text { (3) difficulty concentrating or mind going blank } \\
\text { (4) irritability } \\
\text { (5) muscle tension } \\
\text { (6) sleep disturbance (difficulty falling or staying asleep, or } \\
\text { restless unsatisfying sleep) }\end{array}$ & $\checkmark$ \\
\hline The focus of the anxiety and worry is not confined to features of \\
an Axis I disorder
\end{tabular}


Table 5. DSM-IV-TR Diagnoses at the Beginning and End of Treatment

\begin{tabular}{|c|c|c|c|c|}
\hline & \multicolumn{2}{|c|}{$\begin{array}{l}\text { DSM-IV-TR Diagnosis at the Beginning of } \\
\text { Treatment }\end{array}$} & \multicolumn{2}{|c|}{$\begin{array}{l}\text { DSM-IV-TR Diagnosis at the End } \\
\text { of Treatment }\end{array}$} \\
\hline Axis I & $\begin{array}{l}300.3(\mathrm{~F} 42.2) \\
300.2(\mathrm{~F} 41.1)\end{array}$ & $\begin{array}{l}\text { Obsessive-Compulsive Disorder } \\
\text { Generalized Anxiety Disorder }\end{array}$ & & None \\
\hline Axis II & V71.09 & No Diagnosis & V71.09 & No diagnosis \\
\hline Axis III & & None & & None \\
\hline Axis IV & & None & & None \\
\hline Axis V & & $\mathrm{GAF}=45$ & & $\mathrm{GAF}=80$ \\
\hline
\end{tabular}

Table 6. Sample Generic Session Structure Provided in March, Mulle, Foa, \& Kozak's (2002)

Treatment of Pediatric Obsessive-Compulsive Disorder:

Cognitive Behavioral Therapy Treatment Manual

\begin{tabular}{|l|}
\hline Session Tasks \\
\hline Check in \\
\hline Review homework \\
\hline Teaching/learning tasks for week \\
\hline $\begin{array}{l}\text { Therapist-assisted practice (e.g., in-vivo or } \\
\text { imaginal exposures) }\end{array}$ \\
\hline Discuss and agree upon homework \\
\hline
\end{tabular}


Table 7. Sample Agenda/Plan from Session 9

Session 9 - 3/28/16

- Review symptoms and functioning past week

- Completing exposures/monitoring - not doing the monitoring every day

- Why not? Need to continue to problem solve

- Ritual prevention -> resisting more - Why is it important to do this?

- Re-exposure to stimuli if he ritualizes - how did this go?

- Continue reducing avoidance - going to stores!

- Exposure

- What are things that you avoid touching?

- Face exposure?

- 1) Hardest thing and then touching face - need to reduce avoidance on this

- Brainstorm and decide upon

- Bring parents into session - Return to parent involvement/accommodation of his OCD

- Avoidance: Helping him avoid "contaminated" things

- Reassurance

- Engagement in rituals - needing to clean anything that he thinks is dirty

- Return to discussion of 2 sessions per week

- In person or via remote video?

- Plan HW Exposure "Menu" \& Ritual Prevention Goal Percentage 
Pragmatic Case Studies in Psychotherapy, http://pcsp.libraries.rutgers.edu

Volume 15, Module 1, Article 1, pp. 1-74, 03-22-19 [copyright by author]

Table 8. Sample Online Homework Monitoring Form from the Week Preceding Session 9 (3/28/16)

\begin{tabular}{|c|c|c|c|c|c|c|c|c|c|c|}
\hline $\begin{array}{l}\text { Target for this } \\
\text { week } \rightarrow>\end{array}$ & $\begin{array}{l}\text { Menu: Remote control, } \\
\text { touching walls, or putting } \\
\text { up toilet seat lid - } 20 \\
\text { seconds touching - then } \\
\text { rubbing contaminated } \\
\text { hands all over clothes and } \\
\text { arms/legs/neck/etc }\end{array}$ & $\begin{array}{l}\text { Remember: } \\
\text { after doing a } \\
\text { ritual, } \\
\text { RE-EXPOSE! }\end{array}$ & 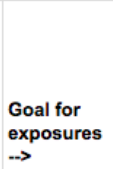 & $\begin{array}{l}\text { at least } 40 \\
\text { mins }\end{array}$ & $\begin{array}{l}\text { When Daniel } \\
\text { ritualizes (\# } \\
\text { times washed } \\
\text { hands, } \\
\text { showered, } \\
\text { cleaned) }\end{array}$ & $\begin{array}{l}\text { When Daniel } \\
\text { successfully } \\
\text { resists } \\
\text { ritualizing (\# } \\
\text { times resisted } \\
\text { urge to } \\
\text { wash/clean) }\end{array}$ & & & & \\
\hline Day & Situation/Trigger & Temp 1-10 & \begin{tabular}{|l|} 
Bossed \\
back \\
successful \\
|y? Yes/No \\
\end{tabular} & $\begin{array}{l}\text { If resist } \\
\text { longer. } \\
\text { how long? }\end{array}$ & OCD wins & DB Wins & \% DB Won & Goal \% & Comments: & Alex: \\
\hline Tues $3 / 22$ & Remote control & 5 & $\mathrm{Y}$ & No & 8 & 1 & $11 \%$ & $30 \%$ & $\begin{array}{l}\text { I (mom) watched } \\
\text { Daniel catch himelf } \\
\text { before he washed } \\
\text { his hands. He } \\
\text { stopped and smiled! } \\
\text { Great to see! }\end{array}$ & $\begin{array}{l}\text { Awesome! } \\
\text { Let's keep } \\
\text { practicing } \\
\text { this type of } \\
\text { resistance - } \\
\text { Alex }\end{array}$ \\
\hline Wed $3 / 23$ & Remote control & 5 & $\mathrm{Y}$ & No & 8 & 2 & $20 \%$ & $30 \%$ & & \\
\hline Thurs $3 / 24$ & Remote control & 6 & $\mathrm{Y}$ & No & 7 & 3 & $30 \%$ & $30 \%$ & & \\
\hline Friday $3 / 25$ & Remote control & 7 & $\mathrm{Y}$ & No & 8 & 2 & $20 \%$ & $30 \%$ & & \\
\hline Saturday $3 / 26$ & Remote control & 5 & $Y$ & No & 7 & 2 & $22 \%$ & $30 \%$ & & \\
\hline Sunday $3 / 27$ & Remote control & 5 & $Y$ & No & 7 & 4 & $36 \%$ & $30 \%$ & $\begin{array}{l}\text { Went to } 4 \text { stores } \\
\text { today! }\end{array}$ & $\begin{array}{l}\text { WOW! Great } \\
\text { job Daniel! }\end{array}$ \\
\hline
\end{tabular}

Table 9. Post-Session Clinical Reflection Notes

Session 9 - 3/28/16 - at the office

- Longer than 60 minutes, closer to 90

- Continue with exposures -> he was avoiding touching his face, so still need to go after this

- Review parent involvement

- Mom felt bad, she felt like she was doing something wrong and that she had to "fix" it - normalized but also pushed her

- Also changed rewards to directly reward completing exposures and

- Tx planning: going up to 2 sessions per week?

- Going 2x/wk - remote video at first - doesn't want to travel down with his Dad, whom he said is not as "protective" as his mother

- Other things that came up in session:

- Went to stores! Motivated by getting game

- Make sign for sink as reminder to not wash, or to re-expose

- Questions for Supervision w/Marty, 3/31/16

- How to encourage increased difficulty with exposures?

- Mainly what should I be expecting of Daniel in terms of difficulty?

- I.e. \# of exposures, or level of difficulty

- How to communicate that to him? 
Pragmatic Case Studies in Psychotherapy, http://pcsp.libraries.rutgers.edu

Volume 15, Module 1, Article 1, pp. 1-74, 03-22-19 [copyright by author]

Table 10. Course of Treatment: Additional Session Information

\begin{tabular}{|c|c|c|c|c|c|c|}
\hline Session \# & Date & $\begin{array}{c}\text { Days } \\
\text { Since } \\
\text { Last } \\
\text { Session }\end{array}$ & $\begin{array}{l}\text { Session } \\
\text { Length } \\
\text { (Mins) }\end{array}$ & $\begin{array}{c}\text { Who Attended } \\
\text { Session? }\end{array}$ & $\begin{array}{c}\text { Office or } \\
\text { Remote } \\
\text { Video }\end{array}$ & $\begin{array}{l}\text { Principle } \\
\text { vs } \\
\text { Protocol }\end{array}$ \\
\hline Intake & $11 / 16 / 15$ & - & 120 & $\begin{array}{c}\text { Daniel \& Both } \\
\text { Parents }\end{array}$ & Office & Assessment \\
\hline 1 & $1 / 25 / 16$ & - & 75 & $\begin{array}{c}\text { Daniel \& Both } \\
\text { Parents }\end{array}$ & Office & Assessment \\
\hline 2 & $2 / 1$ & 7 & 60 & $\begin{array}{c}\text { Daniel \& Both } \\
\text { Parents }\end{array}$ & Office & Assessment \\
\hline 3 & $2 / 8$ & 7 & 60 & Daniel Only & Office & Protocol \\
\hline 4 & $2 / 15$ & 7 & 60 & Daniel Only & Office & Protocol \\
\hline 5 & $2 / 22$ & 7 & 60 & $\begin{array}{c}\text { Daniel \& Both } \\
\text { Parents } \\
\end{array}$ & Office & Protocol \\
\hline 6 & $2 / 29$ & 7 & 105 & $\begin{array}{c}\text { Daniel \& Both } \\
\text { Parents }\end{array}$ & Office & Principle \\
\hline 7 & $3 / 7$ & 7 & 75 & $\begin{array}{c}\text { Daniel \& Both } \\
\text { Parents }\end{array}$ & Office & Principle \\
\hline 8 & $3 / 21$ & 14 & 105 & $\begin{array}{c}\text { Daniel \& Both } \\
\text { Parents }\end{array}$ & Office & Principle \\
\hline 9 & $3 / 28$ & 7 & 90 & $\begin{array}{c}\text { Daniel \& Both } \\
\text { Parents } \\
\end{array}$ & Office & Principle \\
\hline 10 & $3 / 31$ & 3 & 45 & Daniel Only & $\begin{array}{c}\text { Remote } \\
\text { Video }\end{array}$ & Principle \\
\hline 11 & $4 / 4$ & 4 & 60 & $\begin{array}{c}\text { Daniel \& Both } \\
\text { Parents }\end{array}$ & Office & Principle \\
\hline 12 & $4 / 7$ & 3 & 45 & Daniel Only & $\begin{array}{l}\text { Remote } \\
\text { Video }\end{array}$ & Principle \\
\hline 13 & $4 / 14$ & 7 & 75 & Daniel Only & Office & Principle \\
\hline 14 & $4 / 18$ & 4 & 90 & Daniel Only & Office & Principle \\
\hline 15 & $4 / 21$ & 3 & 45 & Daniel Only & $\begin{array}{c}\text { Remote } \\
\text { Video }\end{array}$ & Principle \\
\hline 16 & $5 / 2$ & 11 & 90 & $\begin{array}{c}\text { Daniel \& Both } \\
\text { Parents }\end{array}$ & Office & Principle \\
\hline 17 & $5 / 5$ & 3 & 45 & Daniel Only & $\begin{array}{c}\text { Remote } \\
\text { Video }\end{array}$ & Principle \\
\hline 18 & $5 / 9$ & 4 & 90 & $\begin{array}{c}\text { Daniel \& Both } \\
\text { Parents }\end{array}$ & Office & Principle \\
\hline 19 & $5 / 16$ & 7 & 90 & $\begin{array}{c}\text { Daniel \& Father } \\
\text { Only }\end{array}$ & Office & Principle \\
\hline
\end{tabular}


A.M.B. Tice

Pragmatic Case Studies in Psychotherapy, http://pcsp.libraries.rutgers.edu

Volume 15, Module 1, Article 1, pp. 1-74, 03-22-19 [copyright by author]

Table 10. Course of Treatment: Additional Session Information (continued)

\begin{tabular}{|c|c|c|c|c|c|c|}
\hline Session \# & Date & $\begin{array}{c}\text { Days } \\
\text { Since } \\
\text { Last } \\
\text { Session }\end{array}$ & $\begin{array}{c}\text { Session } \\
\text { Length } \\
\text { (Mins) }\end{array}$ & $\begin{array}{c}\text { Who Attended } \\
\text { Session? }\end{array}$ & $\begin{array}{c}\text { Office or } \\
\text { Remote } \\
\text { Video }\end{array}$ & $\begin{array}{c}\text { Principle } \\
\text { vs } \\
\text { Protocol }\end{array}$ \\
\hline 20 & $5 / 23$ & 7 & 90 & $\begin{array}{l}\text { Daniel \& Both } \\
\text { Parents }\end{array}$ & Office & Principle \\
\hline 21 & $5 / 26$ & 3 & 45 & Daniel Only & $\begin{array}{l}\text { Remote } \\
\text { Video }\end{array}$ & Principle \\
\hline 22 & $6 / 2$ & 7 & 75 & $\begin{array}{c}\text { Daniel \& Father } \\
\text { Only }\end{array}$ & Office & Principle \\
\hline 23 & $6 / 13$ & 11 & 75 & Client \& Parents & Office & Protocol \\
\hline 24 & $7 / 18$ & 35 & 45 & Client \& Parents & Office & Protocol \\
\hline
\end{tabular}


A.M.B. Tice

Pragmatic Case Studies in Psychotherapy, http://pcsp.libraries.rutgers.edu

Volume 15, Module 1, Article 1, pp. 1-74, 03-22-19 [copyright by author]

Figure 1. Daniel's Scores: Children's Yale-Brown Obsessive-Compulsive Scale

\section{Daniel's CY-BOCS Total Severity Ratings}

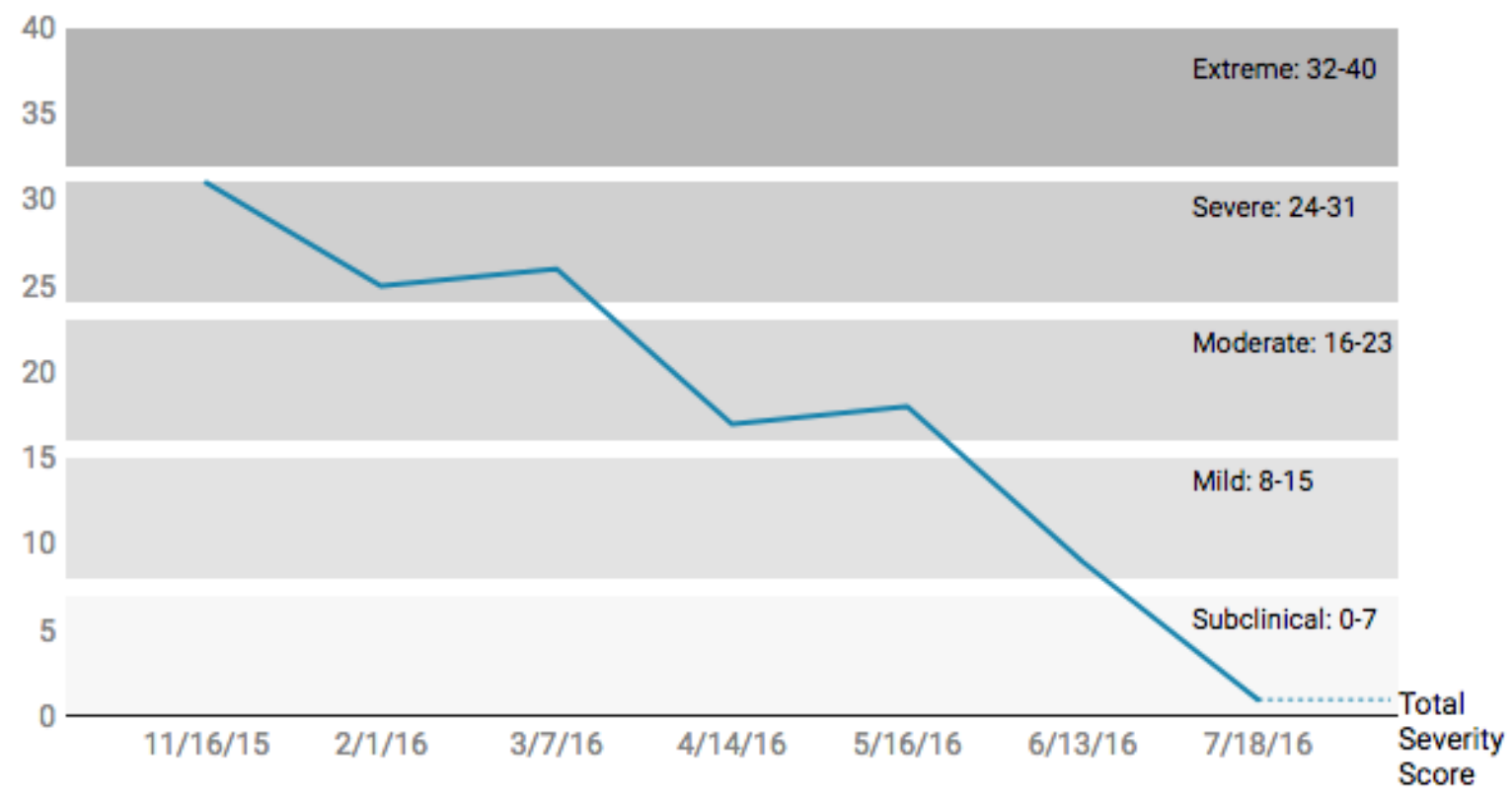

Chart Alexander Tice $\cdot$ Created with Datawrapper 
Pragmatic Case Studies in Psychotherapy, http://pcsp.libraries.rutgers.edu

Volume 15, Module 1, Article 1, pp. 1-74, 03-22-19 [copyright by author]

Figure 2. Daniel's Scores: Severity Measure for Generalized Anxiety Disorder - Child Age 11-17 - DSM-5

\section{Severity Measure for Generalized Anxiety Disorder - Child Age 11-17 (DSM-5)}

4

4

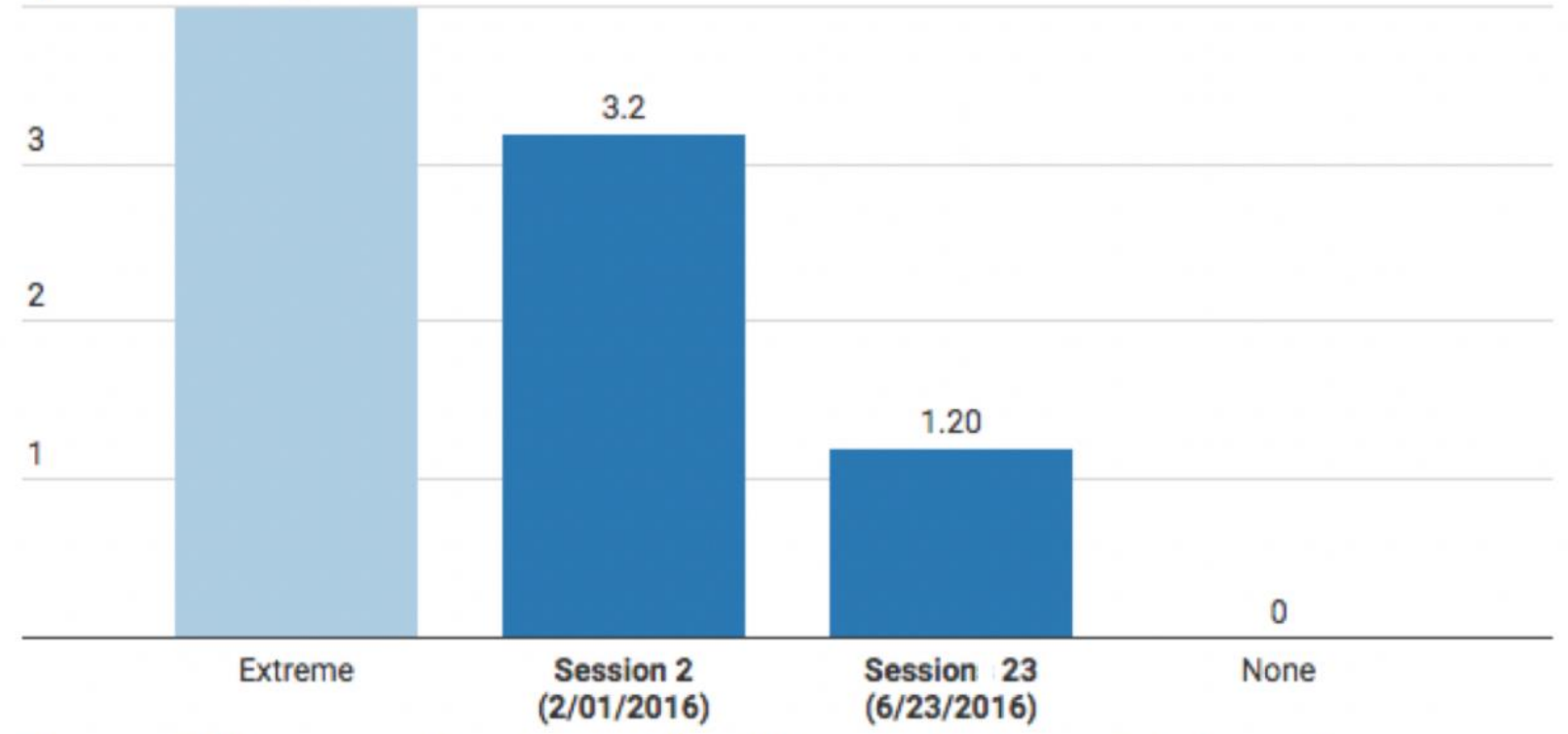

*Average Total Score ranges from 0-4: none (0), mild (1), moderate (2), severe (3), extreme (4) Chart: Alexander Tice $\cdot$ Created with Datawrapper 
Pragmatic Case Studies in Psychotherapy, http://pcsp.libraries.rutgers.edu

Volume 15, Module 1, Article 1, pp. 1-74, 03-22-19 [copyright by author]

Figure 3. Daniel's Scores: Average Completed Rituals

\section{Average Number of Daniel's Daily Rituals}

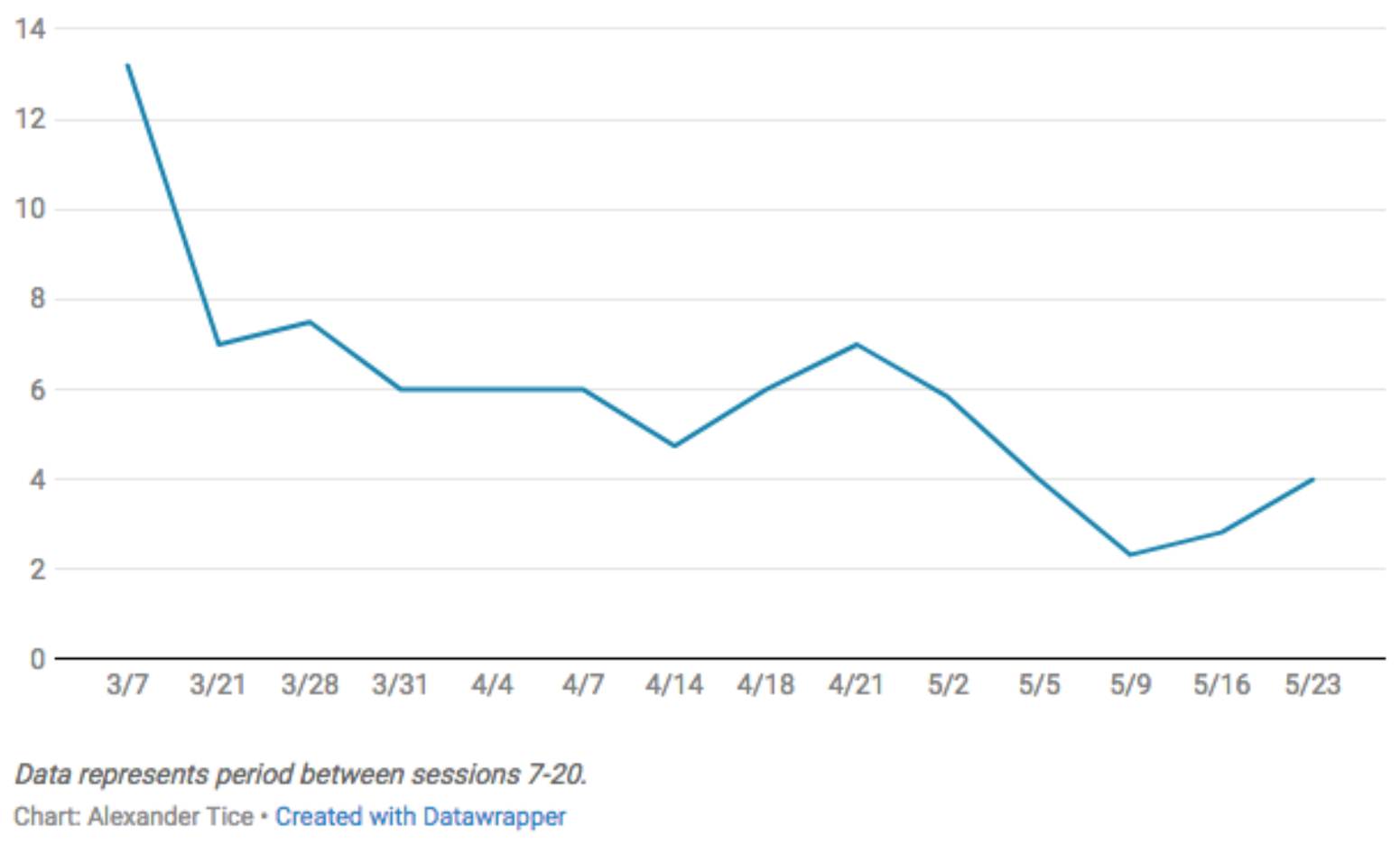


Pragmatic Case Studies in Psychotherapy, http://pcsp.libraries.rutgers.edu

Volume 15, Module 1, Article 1, pp. 1-74, 03-22-19 [copyright by author]

Figure 4. Daniel's Scores: Average Daily Instances of Successful Ritual Prevention

\section{Daniel's Average Daily Successful Ritual Preventions}

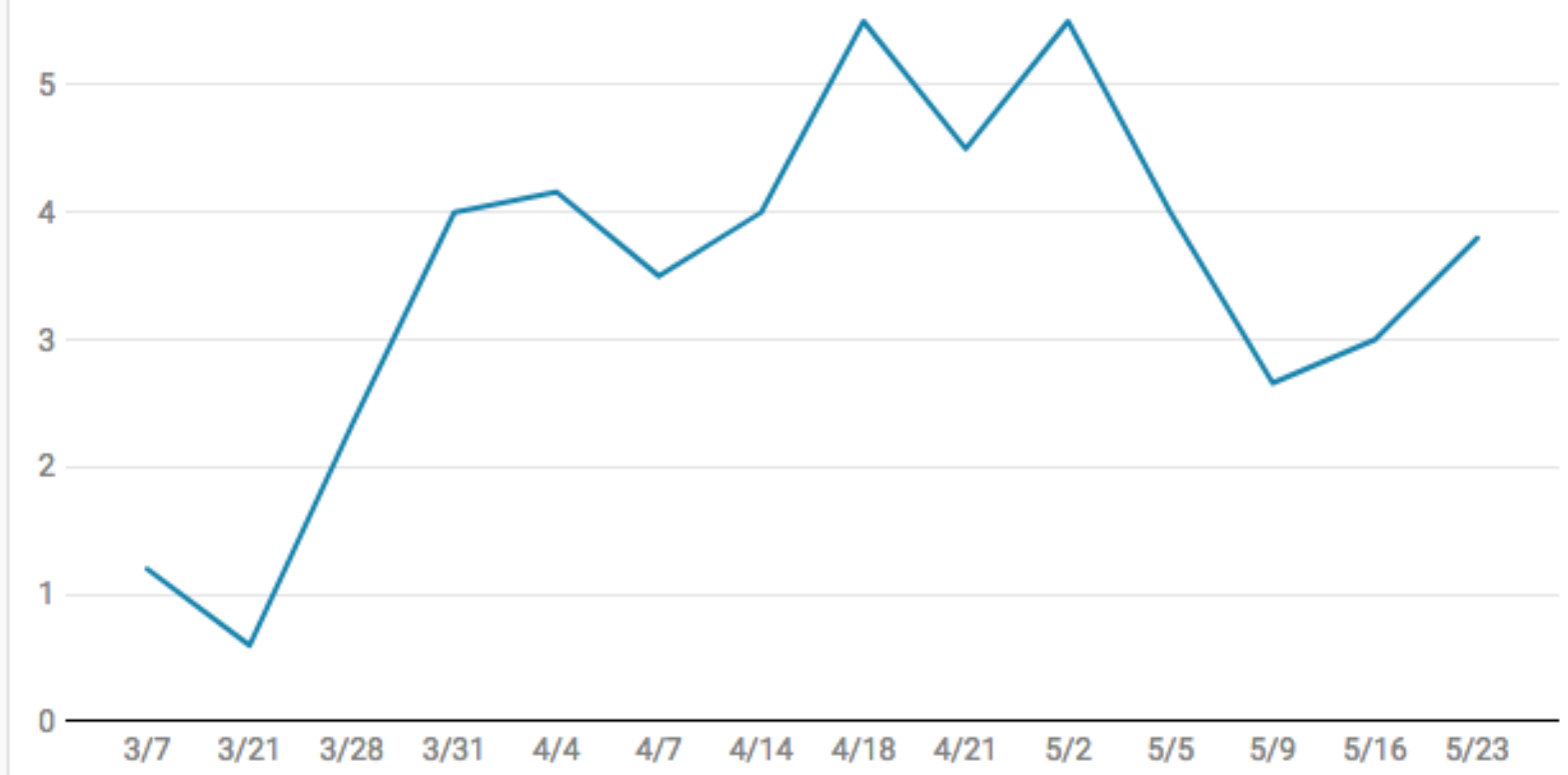

Data represents period between sessions 7-20.

Chart: Alexander Tice * Created with Datawrapper 
A.M.B. Tice

Pragmatic Case Studies in Psychotherapy, http://pcsp.libraries.rutgers.edu

Volume 15, Module 1, Article 1, pp. 1-74, 03-22-19 [copyright by author]

Figure 5. Daniel's Average Successful Ritual Prevention Percentage Versus Goal Percentage

\section{Daniel's Average Successful Ritual Prevention v. Goal}

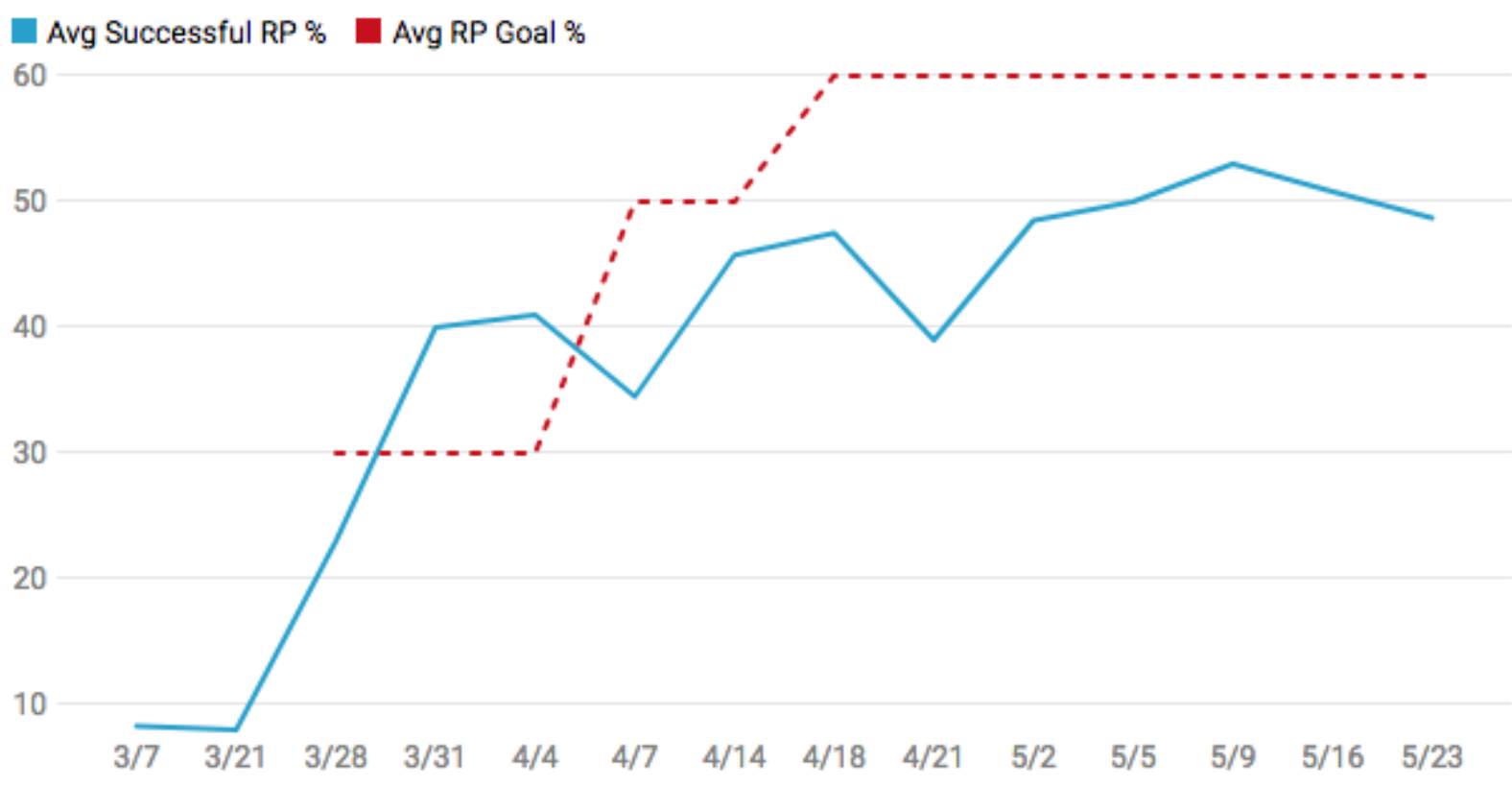

Data represents period between sessions 7-20.

Chart: Alexander Tice $\cdot$ Created with Datawrapper 